\title{
Design of a neutrino source based on beta beams
}

\author{
E. Wildner, C. Hansen, E. Benedetto, E. Jensen, T. Stora, \\ T. Melo Mendonca, and V. Vlachoudis \\ CERN, Geneva, Switzerland \\ E. Bouquerel \\ Institut Pluridisciplinaire Hubert Curien, Université de Strasbourg, \\ CNRS/IN2P3, Strasbourg, France
}

M. Marie-Jeanne, P. Balint, C. Fourel, J. Giraud, J. Jacob, T. Lamy, L. Latrasse, P. Sortais, and T. Thuillier

Laboratoire de Physique Subatomique et de Cosmologie, Université Joseph Fourier Grenoble,

CNRS/IN2P3, Institut National Polytechnique de Grenoble, Grenoble, France

S. Mitrofanov, M. Loiselet, Th. Keutgen, and Th. Delbar

Université catholique de Louvain, Centre de Recherche du Cyclotron,

Louvain-la-Neuve, Belgium

F. Debray, C. Trophime, S. Veys, and C. Daversin

Laboratoire National des Champs Magnétiques Intenses, CNRS, Université Joseph Fourier, Grenoble, France

V. Zorin, I. Izotov, and V. Skalyga

Institute of Applied Physics, Nizhny Novgorod, Russia

A. Chancé and J. Payet

CEA, IRFU, SACM, Gif-Sur-Yvette, France

G. Burt and A. C. Dexter

Lancaster University, Lancaster, United Kingdom

V. L. Kravchuk, ${ }^{*}$ T. Marchi, ${ }^{\dagger}$ M. Cinausero, F. Gramegna,

G. De Angelis, and G. Prete

INFN, Laboratori Nazionali di Legnaro, Legnaro, Italy

G. Collazuol and M. Mezzetto

Physics Department, Padova University and Istituto Nazionale di Fisica Nucleare, Padova, Italy

E. Vardaci, A. Di Nitto, A. Brondi, G. La Rana,

R. Moro, and V. Palladino

Dipartimento di Scienze Fisiche dell'Universitá di Napoli and Istituto Nazionale di Fisica Nucleare, Napoli, Italy

N. Gelli

Istituto Nazionale Fisica Nucleare, Sezione di Firenze, Sesto Fiorentino, Firenze, Italy

M. Mazzocco and C. Signorini

Dipartimento di Fisica dell'Universitá di Padova and Istituto Nazionale di Fisica Nucleare, Padova, Italy

T. Y. Hirsh

Weizmann Institute of Science, Rehovot, Israel, and Soreq NRC, Yavne, Israel

M. Hass

Weizmann Institute of Science, Rehovot, Israel 


\author{
D. Berkovits \\ Soreq NRC, Yavne, Israel
}

\begin{abstract}
A. Stahl, M. Schaumann, and J. Wehner
Aachen University, Aachen, Germany
\end{abstract}

(Received 15 April 2014; published 23 July 2014)

\begin{abstract}
"Beta beams" produce collimated pure electron (anti)neutrino beams by accelerating beta active ions to high energies and having them decay in a racetrack shaped storage ring of $7 \mathrm{~km}$ circumference, the decay ring. EUROnu beta beams are based on CERN infrastructures and existing machines. Using existing machines may be an advantage for the cost evaluation, but will also constrain the physics performance. The isotope pair of choice for the beta beam is ${ }^{6} \mathrm{He}$ and ${ }^{18} \mathrm{Ne}$. However, before the EUROnu studies one of the required isotopes, ${ }^{18} \mathrm{Ne}$, could not be produced in rates that satisfy the needs for physics of the beta beam. Therefore, studies of alternative beta emitters, ${ }^{8} \mathrm{Li}$ and ${ }^{8} \mathrm{~B}$, with properties interesting for a beta beam have been proposed and have been studied within EUROnu. These alternative isotopes could be produced by using a small storage ring, in which the beam traverses a target, creating the ${ }^{8} \mathrm{Li}$ and ${ }^{8} \mathrm{~B}$ isotopes. This production ring, the injection linac and the target system have been evaluated. Measurements of the cross section of the reactions to produce the beta beam isotopes show interesting results. A device to collect the produced isotopes from the target has been developed and tested. However, the yields of ${ }^{8} \mathrm{Li}$ and ${ }^{8} \mathrm{~B}$, using the production ring for production of ${ }^{8} \mathrm{Li}$ and ${ }^{8} \mathrm{~B}$, is not yet, according to simulations, giving the rates of isotopes that would be needed. Therefore, a new method of producing the ${ }^{18} \mathrm{Ne}$ isotope has been developed and tested giving good production rates. A $60 \mathrm{GHz}$ ECRIS prototype, the first in the world, was developed and tested for ion production with contributions from EUROnu. The decay ring lattices for the ${ }^{8} \mathrm{Li}$ and ${ }^{8} \mathrm{~B}$ have been developed and the lattice for ${ }^{6} \mathrm{He}$ and ${ }^{18} \mathrm{Ne}$ has been optimized to ensure the high intensity ion beam stability.
\end{abstract}

DOI: $10.1103 /$ PhysRevSTAB.17.071002

PACS numbers: 25.55.-e, 29.30.Hs, 25.70.Hi

\section{OVERVIEW}

The idea to produce pure neutrinos or pure antineutrinos from beta decay of radioactive isotopes circulating in a racetrack shaped storage ring originated in 2002 [1]. Beta beams are based on the acceleration of beta active isotopes. These isotopes decay in the decay ring and produce, after the long straight sections, pure $\nu_{e}$ or $\bar{\nu}_{e}$ beams depending on whether the accelerated isotope is a $\beta^{+}$or a $\beta^{-}$emitter. The fact that only electron (anti)neutrinos are produced in the reaction is interesting for physics; no other types of neutrinos are present in the production and will therefore not interfere with the wanted reactions in the detector. The neutrino energy depends on the reaction energy value, $Q$, of the beta decay and of the chosen relativistic $\gamma$ boost of the stored isotopes. The neutrino spectrum is well known from the electron spectrum. Basic aspects of the beta beam facility and of the physics of the beta beam can be found in [2].

\footnotetext{
"Also at National Research Center "Kurchatov Institute," Moscow, Russia.

Kravchuk_VL@nrcki.ru

Also at Physics Department, Padova University, Padova, Italy.

Published by the American Physical Society under the terms of the Creative Commons Attribution 3.0 License. Further distribution of this work must maintain attribution to the author(s) and the published article's title, journal citation, and DOI.
}

Isotopes decaying with higher $Q$-value give higher neutrino energy, $E$, compared to the isotopes with lower reaction $Q$-values when accelerated to the same $\gamma$ boost: $E<2 \gamma Q$. Higher energy of the parent beam decreases emission angle in the forward direction since the angle is $1 / \gamma$. Higher neutrino energy requires a further detector, which reduces the flux in the detector, on the other hand the cross section is better at higher energies. To design a beta beam facility consequently means taking into account also the neutrino interaction cross sections, and the neutrino beam divergence from the $\gamma$ boost of the ions. From this we can estimate a merit factor of $\gamma / Q$ for the beta beam flux. Taking this into account, if we choose to increase the neutrino energy by increasing the $Q$-value of the chosen radioactive isotopes, the required neutrino fluxes from the accelerators will increase approximately with $Q$ due to the fact that we need a longer baseline for the physics reach. As exptkllained, by choosing higher $\gamma$ boosts, the beam divergence is smaller and the flux of neutrinos in the detector would be better; on the other hand, the longer decay times at higher $\gamma$ require higher circulating currents in the decay ring or longer straight sections. It will also increase the cost of the accelerator which depends to a large extent on the decay ring size. Decays are not useful in the arcs, therefore arcs should be as small as possible; this has to be weighted with different aspects of available or R\&D magnet technologies for a smaller arc. 
The selection of one of the isotope pairs is mainly guided by production rates and the energy of the neutrino beam from the decay ring, which is also related to what can be practically achieved with the chosen decay ring design. There are several considerations for the choice of a suitable beta emitter isotope. The lifetime of the beta beam isotopes should be such that we get a sufficient number of decays at high relativistic $\gamma$, in the decay ring, but as few as possible at the beginning of the acceleration, where decay times are shorter (the relativistic $\gamma$ is low) and the decays not useful. The optimum overall lifetime is given by the chosen acceleration scenario and is usually in the order of a second in the ion rest frame. Extraction of the ions from the production target and their transport into the charge breeder [electron cyclotron resonance (ECR) ion source] have to be fast to limit the decay losses. Isotopes generating hazardous waste products that cannot be safely handled either at the production phase or after decay, would not be an acceptable choice. Efficient isotope production giving a sufficient amount of radioactive ions for acceleration is one of the important challenges for beta beams.

Noble gases are chemically stable and therefore good candidates for beta beams. The charge-to-mass ratio of the ions has to be large enough for efficient acceleration. Highly charged ions induce space-charge phenomena that have unwanted effects on the beam properties. A specific accelerator can accelerate fully stripped ions up to $Z / A$ times its maximum proton energy, where $A$ is the total number of nucleons and $Z$ is the atomic number of the ion. New isotope production and extraction methods have to be specifically developed for beta beams.

Two isotope pairs with the required properties have been selected for studying a beta beam facility: ${ }^{6} \mathrm{He} /{ }^{18} \mathrm{Ne}$ and ${ }^{8} \mathrm{Li} /{ }^{8} \mathrm{~B}$ for $\bar{\nu}_{e} / \nu_{e}$ production. ${ }^{6} \mathrm{He}$ and ${ }^{18} \mathrm{Ne}$ have $Q$-values of 3.5 and $3.3 \mathrm{MeV}$, and decay times at rest of 0.8 and $1.67 \mathrm{~s}$ respectively; in this context we refer to them as low$Q$ ions. The alternative ion pair, ${ }^{8} \mathrm{Li}$ and ${ }^{8} \mathrm{~B}$, has $Q$-values of 13.0 and $13.9 \mathrm{MeV}$ and the decay times for these isotopes are 0.84 and $0.77 \mathrm{~s}$, respectively. But as the merit factor is $\gamma / Q$, for the high- $Q$ isotope pair, ${ }^{8} \mathrm{Li} /{ }^{8} \mathrm{~B}$, we need about 5 times higher ion intensities for the fact that the detector has to be situated further away for the higher neutrino energy. Collective effects may then contribute considerably to the challenges for beam physics. The alternative, higher parent particle energy, needs a larger decay ring straight section to get the needed rates of decay (longer decay times at higher energies).

CERN has a suitable infrastructure to accelerate ions to high energies. To use existing machines and infrastructure for the acceleration of the beta active isotopes is advantageous, however it also constrains the facility (the machines are not designed for the beta beam and coexistence with other physics programs has to be considered in addition). A chosen accelerator can only accelerate a limited number of charges. The CERN accelerator complex has been used to evaluate the physics of a beta beam. We have not investigated a beta beam in a "green field" scenario; less decay losses by more rapid acceleration and a better distribution of the ions in the machines compared to the CERN option, for example, could be beneficial for the beta beam. The CERN SPS allows a maximum $\gamma$ value of $150\left({ }^{6} \mathrm{He}\right)$ or 250 $\left({ }^{18} \mathrm{Ne}\right)$. The choice of energy, corresponding to a $\gamma$ value of 100 , was made to optimize the physics reach at a baseline $130 \mathrm{~km}$ from CERN where the proposed MEMPHYS [3] detector would be located. This detector in the Fréjus tunnel would be a Mton water Cherenkov detector. In Fig. 1 the layout for the two options ${ }^{6} \mathrm{He} /{ }^{18} \mathrm{Ne}$ and ${ }^{8} \mathrm{Li} /{ }^{8} \mathrm{~B}$ are shown. The high- $Q$ option was proposed as a solution to get the necessary production rates of the needed $\beta^{+}$emitters, producing the neutrinos. However, the low- $Q$ option, ${ }^{6} \mathrm{He} /{ }^{18} \mathrm{Ne}$, is the only beta beam that can be proposed as a possible option today, thanks to research on the production of ${ }^{18} \mathrm{Ne}$.

Neutrino detectors are expensive and detectors are usually shared between accelerator based neutrino physics and other physics, like astrophysics. Most often, the distance the neutrinos have to travel from the point where they are created to their detection is given by the position of a detector (existing or not) relative to an existing accelerator facility. To get the wanted physics reach, the energy, E, of the neutrinos are given by the distance, $\mathrm{L}$, to the detector. So if one wants to use an existing particular detector, or an existing underground mine to install a new detector, the required energy of the neutrino is given. L/E is $500 \mathrm{~km} / \mathrm{GeV}$ at the first oscillation maximum and $1500 \mathrm{~km} / \mathrm{GeV}$ at the second etc. So in the case of the beta beam we have two possible detector locations: the

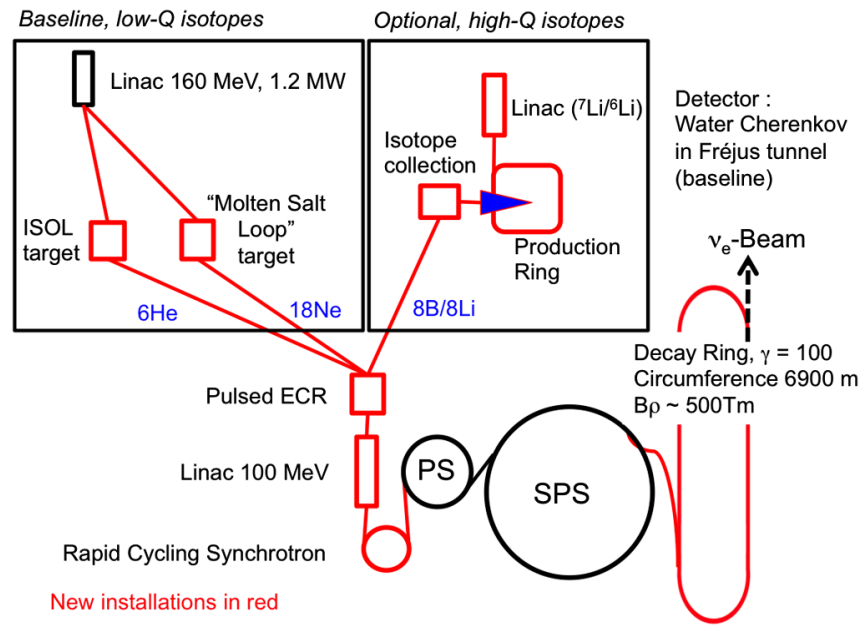

FIG. 1. Layout of the CERN beta beam. Two beta emitting isotope pairs are considered: ${ }^{6} \mathrm{He} /{ }^{18} \mathrm{Ne}$ (low- $Q$ ) and ${ }^{8} \mathrm{Li} /{ }^{8} \mathrm{~B}$ (high- $Q$ ) for $\nu_{e} / \bar{\nu}_{e}$ production respectively. The decay ring has similar dimensions for the two isotope pairs. The inclination angle is $0.6^{\circ}$ for a neutrino beam pointing at the Fréjus detector and $3^{\circ}$ for a neutrino beam from CERN to Gran Sasso in Italy, or Canfranc in Spain. 
Fréjus laboratory in France and a possible detector location in Canfranc or Gran Sasso. The baseline configuration chosen for physics is a Lorenz factor of 100 and the first oscillation maximum, which for low- $Q$ isotopes means that the detector will be in the Fréjus laboratory and for the high- $Q$ case at a distance comparable to CERN-Canfranc. The physics is not identical at different detector distances; these aspects are treated in the EUROnu physics contribution to this issue of PRST-AB.

For an optimal sensitivity of the CERN beta beam facility to the $\theta_{13}$ angle and $\mathrm{CP}$ violating phase, a total throughput of $1.1 \times 10^{19}$ neutrinos and $2.9 \times 10^{19}$ antineutrinos immediately after the decay ring was generally assumed over a running period of 10 years (200 days/year, $50 \%$ efficiency) for the low- $Q$ option. This has been calculated by Monte Carlo simulation [4] to get statistics relevant to the measurement capacities in the detectors. It is necessary to have a comparable "merit factor" (see discussion above) if we vary the $\gamma$ boost or $Q$-value to get the required flux. If we increase the energy by increasing the $Q$-value (changing our isotopes), we need to produce about $\Delta Q$ times more neutrinos. There are in addition several aspects related to different detector types and what can be achieved for physics, but for the purpose of this paper the set of requirements mentioned in this paragraph is our baseline working hypothesis. The top-down approach then results in the need for production of about $3.3 \times 10^{13}{ }^{6} \mathrm{He}$ radioactive atoms and $2.1 \times 10^{13}{ }^{18} \mathrm{Ne}$ atoms per second, taking into account efficiency coefficients along the accelerator chain. Even if the production of ${ }^{18} \mathrm{Ne}$ is a factor of 2 too low, the experiment can run longer for the isotope lacking production and still give good physics reach. Today we know that the oscillation angle $\theta_{13}$ is relatively large ( $\sin$ $2 \theta_{13}$ is determined to be $0.092 \pm 0.016$ (stat) \pm 0.005 (sys) [5]) and the sensitivity of the beta beam to the $C P$ violating phase for this specific value of $\theta_{13}$ is now the important performance measure. If, for large $\theta_{13}$, the suppression factor (the time the neutrino beam is "on" with respect to not having any beam, during the experimental time) in the detector can be relaxed, which seems to be the case, these rates may be increased by a redistribution of the ions in the machines (larger number of less intense bunches).

The beta beam isotopes are accelerated in an ion linac after being collected in a charge breeding ECR source. The ionized isotopes then pass through a rapid cycling synchrotron (RCS) [6], the CERN PS synchrotron and the last acceleration stage before the decay ring (DR) is the CERN SPS. The decay ring [7] would have a circumference of $6900 \mathrm{~m}$ and a straight section length of almost $2700 \mathrm{~m}$. The main bending magnet field is $6 \mathrm{~T}$. Consequently superconducting technology is necessary. The presently studied CERN scenarios are shown in Fig. 1. A considerable part of the efforts spent to make the beta beam a solid option for neutrino production deals with the integration of the beta beam into the CERN accelerator complex. In the baseline option we have taken an upgraded linac 4 [8] as the proton driver also for production of ${ }^{6} \mathrm{He}$; this option is used in the costing analysis. An existing superconducting proton linac (SPL) [9] may be used for the ${ }^{6} \mathrm{He}$ production but it would not be necessary.

Studies of collective effects in the SPS and in the PS have been started. The decay ring work is more advanced. This machine, since not yet constructed, can still be improved and use modern approaches and technology like better impedance control. The PS and the SPS are already today receiving high intensity beams and approach levels of irradiation that need special attention for the longevity of the equipment. Therefore measures are needed to make sure that a beta beam can be integrated in the physics program.

As mentioned above, it is of interest for a neutrino facility to be able to use existing detectors at strategic distances for physics performance. In the present LAGUNA study [10], the Fréjus site is one of the studied options to place a neutrino detector. The beta beam has been laid out on the CERN site for the Fréjus option [11], see Fig. 2. The beam is extracted from the SPS and injected into the decay ring. The ring is oriented so that the neutrino beam is directed towards Fréjus in France. A beta beam facility, using the isotope pair ${ }^{6} \mathrm{He} /{ }^{18} \mathrm{Ne}$ and a baseline from CERN to Fréjus in France has been studied within the European Framework Program 6, the FP6 EURISOL Design Study (2005-2009) [12]. The studied scenario is based on CERN infrastructure and machines and on existing technologies. After the EURISOL Design Study there was an estimated significant deficit in the production of ${ }^{18} \mathrm{Ne}$. The European FP7 EUROnu beta beam proposal was based on a new idea to produce neutrinos from the decay of ${ }^{8} \mathrm{Li}$ and ${ }^{8} \mathrm{~B}$ with an internal target in a production ring [13] to be able to get the required rates of neutrinos. In addition to addressing the too limited production rates of

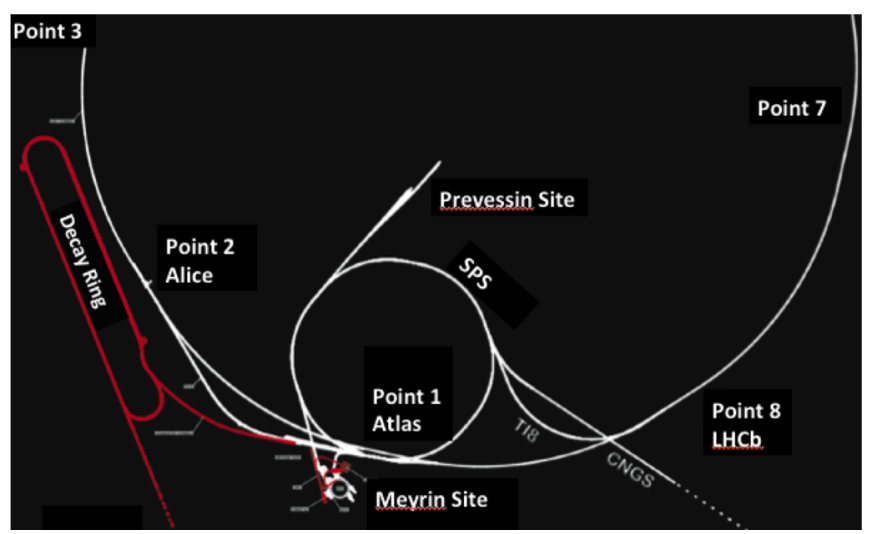

FIG. 2. The CERN beta beam directed to Fréjus, the inclination angle of the decay ring is $0.6^{\circ}$ for a neutrino beam pointing at the Fréjus detector. It is placed close to point 2 of the LHC, of which parts can be seen in the figure, using parts of the extraction lines from the SPS. 
the beta emitters, the EUROnu project made it possible to address some crucial aspects of the beta beam that came out after the FP6 study: end to end simulations of the performance and stability of the high intensity ion beams. Emphasis was also put into the prototyping of the very challenging ECR source to be used for beta beams. The new production concept was studied within EUROnu (see Sec. III A) and the conclusion was that, for the time being, the technology issues are major and a redirection of the research was necessary. Another approach was then put forward during the study, namely to produce the ${ }^{18} \mathrm{Ne}$ isotopes using a molten salt loop target at CERN ISOLDE [14]. Research and measurements ${ }^{18} \mathrm{Ne}$ production rates from the molten salt target, together with the already performed experiments for the production of ${ }^{6} \mathrm{He}$, now makes the option of using the isotope pair ${ }^{6} \mathrm{He} /{ }^{18} \mathrm{Ne}$ the baseline option for the beta beam with the detector in the Fréjus tunnel, $130 \mathrm{~km}$ from CERN. This article describes the work accomplished within the EUROnu collaboration. For completeness some of the already done work on the CERN beta beam is included in the article.

\section{THE PRODUCTION OF LOW-Q RADIOISOTOPES FOR THE BETA BEAMS}

The demonstration of the technical feasibility of the production of the isotopes required for the beta beams has significantly progressed during the past years. Important developments have been made within the EURISOL-DS project [15]. Part of the study was dedicated to the production of the isotope pair ${ }^{6} \mathrm{He} /{ }^{18} \mathrm{Ne}$, otherwise known as baseline ions, via the isotope separation on-line method (ISOL) [16].

Different production schemes have been proposed depending on the ion type. The production of ${ }^{6} \mathrm{He}$ has been successfully validated using the isotope separation online (ISOL) method [16], where the ions have been obtained with fast neutrons on a thick beryllium oxide target. Experimental tests performed at CERN-ISOLDE showed the validation of the production of $10^{14}{ }^{6} \mathrm{He} / \mathrm{s}$ with $100 \mu \mathrm{A}, 1.4 \mathrm{GeV}$ protons and an optimized geometry [17].

The production of the required $10^{13}{ }^{18} \mathrm{Ne} / \mathrm{s}$ was found to be more challenging and, thus, an alternative route consisting of a circulating loop of molten salt has been proposed [14]. Proton beams close to $1 \mathrm{MW}$ power, from an upgraded Linac4 at CERN, would impinge a circulating molten NaF-based salt to produce extracted rates of $10^{13}$ ${ }^{18} \mathrm{Ne} / \mathrm{s}$. A first test on the feasibility of the production of ${ }^{18} \mathrm{Ne}$ has been performed at CERN-ISOLDE using a standard static target unit.

A new variant of a production scheme for light radioactive beams has been developed, using a high current deuteron beam and a sequential two-target irradiation. The primary target is essentially a neutron converter, providing a fast and possibly directed neutron source while the actual production takes place separately in a secondary target by fast neutron induced reactions. The efficiency of this setup results from complete separation of the two most major problems in radioactive ion beam production, namely, heat removal of the beam power and extraction of the radioisotopes from the target material.

$\mathrm{By}$ using porous, microfiber target materials, $\mathrm{BeO}$ for ${ }^{6} \mathrm{He}$ and $\mathrm{B}_{4} \mathrm{C}$ for ${ }^{8} \mathrm{Li}$ production, respectively, high yields of these isotopes can be produced. This technique is also easily scalable and can thus serve as a firm basis for the utilization of ${ }^{6} \mathrm{He}$ and ${ }^{8} \mathrm{Li}$ as prime candidates for the "beta-beam" concept.

A review of the progress achieved in the production of the baseline ions will be given in the following subsections.

\section{A. Production of high intensity ${ }^{6} \mathrm{He}$ beams}

The production of ${ }^{6} \mathrm{He}$ is obtained with fast neutrons on a beryllium oxide target through the ${ }^{9} \mathrm{Be}(\mathrm{n}, \alpha)^{6} \mathrm{He}$ reaction, which benefits from high cross sections over a wide neutron energy spectrum [18]. Neutrons in the $0.1-10 \mathrm{MeV}$ range, of interest for ${ }^{6} \mathrm{He}$ production, are to a first approximation emitted in all directions from solid metal converters that will act as neutron spallation sources. Therefore, a conceptual layout of a dual converter-target assembly has been proposed. As shown in Fig. 3(a), the assembly is composed by a cylinder made of tungsten or tantalum in the center of a concentric beryllium oxide production target. In addition, this layout has been adapted to integrate a mechanical support and water-cooling circuit to the converter in order to accommodate a beam of $100 \mathrm{~kW}, 1 \mathrm{GeV}$ protons. Figure 3(b) shows a preliminary configuration which integrates these different elements.

The validation of the required ${ }^{6} \mathrm{He}$ intensities for the $\beta$ beams has been performed with on-line tests at CERNISOLDE [17]. These tests have been performed using a standard ISOLDE unit, as shown in Fig. 4, which consisted of a tungsten neutron converter placed next to a cylindrical target oven containing beryllium oxide target material. This assembly is a simplified version of the optimized geometry developed for the beta beams shown in Fig. 3(a). The target material was composed by porous, small grained beryllium oxide sintered pellets of density of $2.1 \mathrm{~g} / \mathrm{cm}^{3}$. The pellets have been stacked in a standard $20 \mathrm{~cm}$ long and $2 \mathrm{~cm}$ diameter oven, which has been further connected to a versatile arc discharge ion source (VADIS) [20] via a watercooled transfer line.

The operation parameters, release properties and production ${ }^{6} \mathrm{He}$ yields have been monitored with pulsed $1.4 \mathrm{GeV}$ protons delivered from the proton synchrotron booster (PSB) accelerator. The extraction efficiency and deduced yields have been measured as a function of the target temperature in a range from $700{ }^{\circ} \mathrm{C}$ to $1400^{\circ} \mathrm{C}$. A representative curve showing the release of ${ }^{6} \mathrm{He}$ at $1400^{\circ} \mathrm{C}$ is shown in Fig. 5(a). The shape of the curve originates from the diffusion and effusion processes in the production unit, from the beryllium oxide matrix up to the ion source. 


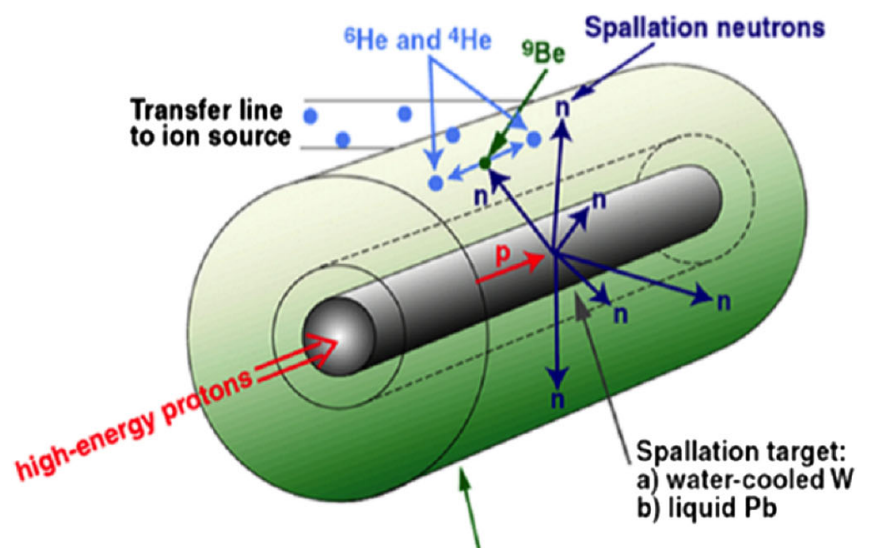

ISOL target $(\mathrm{BeO})$ in concentric cylinder

(a)

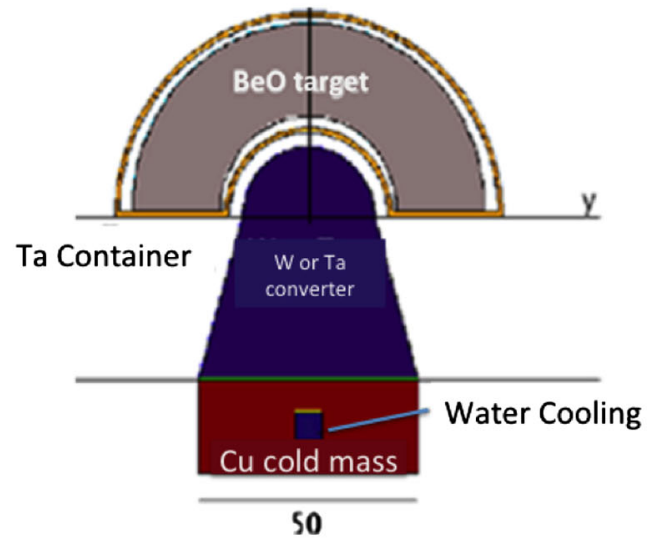

(b)

FIG. 3. (a) Conceptual dual converter-target unit for $1 \mathrm{GeV}$ protons [15]. (b) Unit with first engineering elements [19].

From the shape of the curve, one can observe that ${ }^{6} \mathrm{He}$ is released from the production unit following a rising part and a subsequent decay after the short proton beam impact.

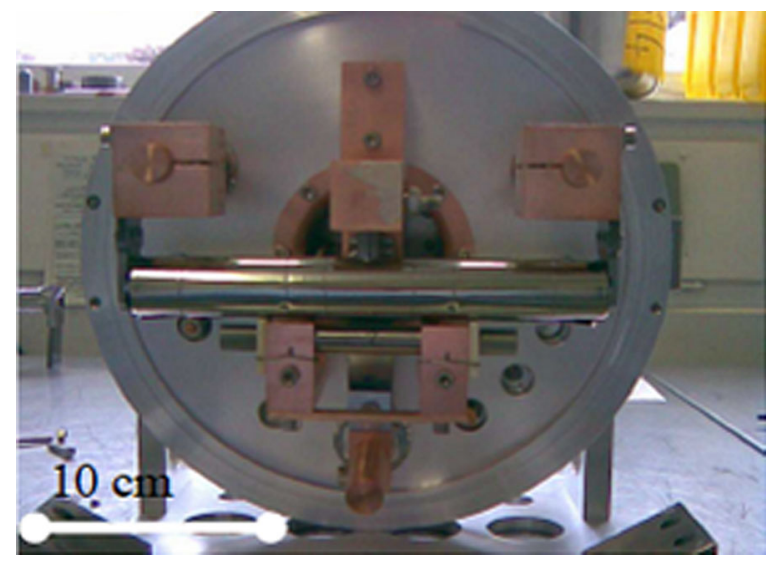

FIG. 4. Configuration of the neutron converter (tungsten cylinder and beryllium oxide target used for on-line tests at CERN-ISOLDE).
The deduced ${ }^{6} \mathrm{He}$ yields [shown in Fig. 5(b)] from the release curves at different target temperatures showed an intarget production that ranged from $2.6 \times 10^{10}$ to $4.1 \times 10^{10}$ ${ }^{6} \mathrm{He} / \mu \mathrm{C}$ of incident protons, in excellent agreement with the calculated $2.8 \times 10^{10}{ }^{6} \mathrm{He} / \mu \mathrm{C}$ and $2.4 \times 10^{10}{ }^{6} \mathrm{He} / \mu \mathrm{C}$ using FLUKA and GEANT4, respectively [17]. Therefore, about $82 \%$ of the produced isotopes were released at a target temperature of $1400{ }^{\circ} \mathrm{C}$, which translates into a release efficiency of $57 \%$ at the foreseen beta beam facility [17]. One shall note that in the beta beams configuration, a similar neutron converter beryllium oxide target layout is proposed, with a water-cooled converter to dissipate the deposited beam power of an incoming $200 \mathrm{~kW}$ proton beam and a larger target allowing the interception of a larger fraction of the emitted neutrons.

\section{B. Production of ${ }^{18} \mathrm{Ne}$ beams}

The production of ${ }^{18} \mathrm{Ne}$ was found to be more challenging. It can be performed by $(p, X)\left(\right.$ or $\left.\left({ }^{3} \mathrm{He}, X\right)\right)$ reaction on $\mathrm{Na}, \mathrm{F}$ or Mg targets [21]. The use of targets containing any of these elements would present several advantages on the

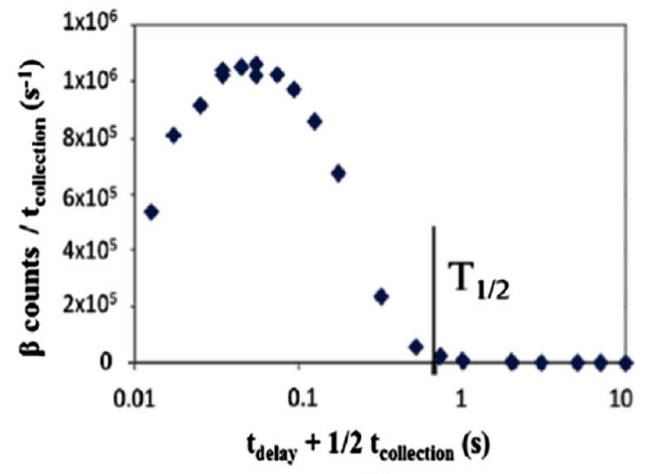

(a)

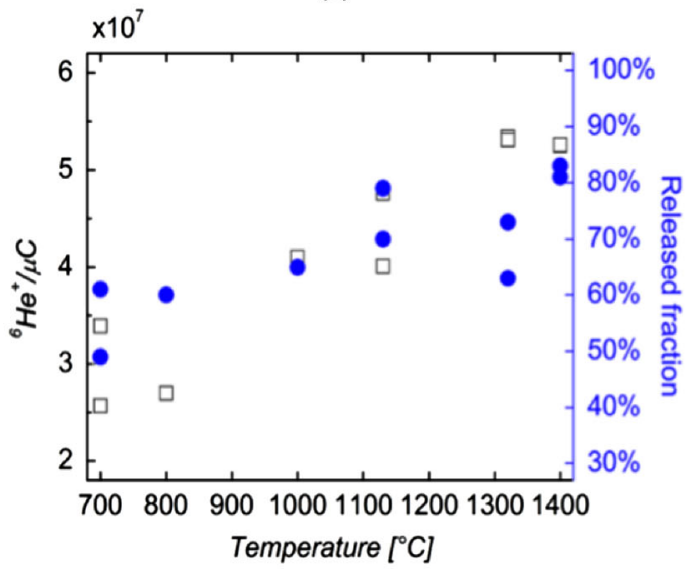

(b)

FIG. 5. (a) Release curve obtained at $1400^{\circ} \mathrm{C}$ : time evolution of ${ }^{6} \mathrm{He}$ isotopes/s (black squares) released from the target unit in function of the time after their production at the proton beam impact. (b) Left side scale: experimentally measured yields (empty squares) for different target temperatures; right side scale: release fraction (full dots) determined from the release curves. 
TABLE I. List of relevant physicochemical properties exhibited by the $\mathrm{NaF}: \mathrm{ZrF}_{4}$ and $\mathrm{NaF}: \mathrm{LiF}$ binary systems: composition, melting point $\left(\mathrm{T}_{\mathrm{M}}\right)$, room temperature density and $900{ }^{\circ} \mathrm{C}$ vapor pressure $\left(\mathrm{P}_{\text {vapor }}\right)$.

\begin{tabular}{lcccc}
\hline \hline Salt & $\begin{array}{c}\text { Composition } \\
(\mathrm{mol} \%)\end{array}$ & $\begin{array}{c}\mathrm{T}_{\mathrm{M}} \\
\left({ }^{\circ} \mathrm{C}\right)\end{array}$ & $\begin{array}{c}\text { Density } \\
\left(\mathrm{g} / \mathrm{cm}^{3}\right)\end{array}$ & $\begin{array}{c}\mathrm{P}_{\text {vapor }} \\
(\mathrm{mm} \mathrm{Hg})\end{array}$ \\
\hline $\mathrm{NaF}: \mathrm{ZrF}_{4}$ & $60: 40$ & 500 & 3.14 & 5 \\
$\mathrm{NaF}: \mathrm{LiF}$ & $39: 61$ & 649 & 2.75 & 0.1 \\
\hline \hline
\end{tabular}

production of Ne. Amongst the wide list of available molten salts, the best candidate to the present application would be sodium fluoride $(\mathrm{NaF})$. However, the high melting point of this salt $\left(995^{\circ} \mathrm{C}\right.$ [22]) limits its applications and the use of a binary system containing $\mathrm{NaF}$ would be more advantageous.

An extensive list of molten salts is available in the literature due to their application as coolants in nuclear reactors $[22,23]$ and more recently in optics and solar cells. Two different binary systems have been first proposed as candidates for the production of ${ }^{18} \mathrm{Ne}: \mathrm{NaF}: \mathrm{ZrF}_{4}$ $(60: 40 \mathrm{~mol} \%)$ and $\mathrm{NaF}: \operatorname{LiF}(39: 61 \mathrm{~mol} \%)$. Both mixtures present eutectics with melting points at $500^{\circ} \mathrm{C}$ and $649^{\circ} \mathrm{C}$, respectively. A summary of relevant physicochemical properties is listed in Table I.

The choice of the salt composition has been made on a basis of thermal stability and low vapor pressure at operating temperatures. One shall note that the low vapor pressures of the salt are not only required to keep the stability of the system but it also avoids the change of the molten salt composition that can occur due to incongruent vaporization. Following these criteria, the systems $\mathrm{NaF}: \mathrm{ZrF}_{4}$ and $\mathrm{NaF}: \mathrm{LiF}$ have been carefully investigated in order to obtain the most adequate salt to the present application.

For the synthesis of both binary systems, high purity $\mathrm{NaF}, \mathrm{ZrF}_{4}$ and $\mathrm{LiF}$ have been used as starting reactants. Due to the high reactivity of the starting materials, all handling was carried out in dry glove boxes, under argon atmosphere, to prevent oxide contaminations. The synthesis of the binary systems was obtained by mixing stoichiometric quantities of the starting reactants, which have been heated up to about $\sim 50{ }^{\circ} \mathrm{C}-100^{\circ} \mathrm{C}$ above the melting points of each system. The composition and stoichiometry of the obtained salts has been carefully controlled using scanning electron microscopy (SEM) coupled to an energy dispersive $\mathrm{x}$-ray (EDS) detector. The melting point of the mixtures has been identified via differential scanning calorimetry (DSC).

Following the synthesis and characterization, several annealing tests have been carried out to test the stability of proposed binary systems. These stability tests showed that the $\mathrm{NaF}: \mathrm{ZrF}_{4}$ system is unstable at the operating temperatures required for the circulating loop operation. This instability is due to high vapor pressure and high reactivity of $\mathrm{ZrF}_{4}$, which leads to losses of material and consequent changes in its composition. In contrast, the lower vapor pressures and reactivity with air exhibited by the NaF: $\mathrm{LiF}$ system proved the suitability of this salt for its use in a circulating loop for the production of ${ }^{18} \mathrm{Ne}$.

The first test to validate the production of ${ }^{18} \mathrm{Ne}$ from a molten $\mathrm{NaF}: \mathrm{LiF}$ salt has been performed using a standard target unit at ISOLDE-CERN. The unit consisted of a $21.6 \mathrm{~cm}$ long and $2 \mathrm{~cm}$ diameter hexacylindrical container made of a special nickel-rich alloy (Haynes 242 [24]). The choice of the container material has been made accounting for the high reactivity and corrosive nature of the fluoride salts at high temperatures. Furthermore, the dimensioning of the salt target container has been performed accounting for the material and heat transfer properties.

The metallic container was equipped with a temperature controlled condensation chimney with a helix allowing the condensation of less volatile elements. The container was filled with the $\mathrm{NaF}: \mathrm{LiF}$ melt up to $3 / 4$ of its volume allowing a free surface for the isotopes to diffuse out of the target. Figure 6 shows a picture of the target unit assembly used at ISOLDE for the molten salt tests. The unit was further connected to a versatile arc discharge ion source (VADIS) [20] via a temperature controlled transfer line, suited for the production of noble gases.

The release properties and production yields of ${ }^{18} \mathrm{Ne}$ have been assessed at CERN-ISOLDE with $1.4 \mathrm{GeV}$ from

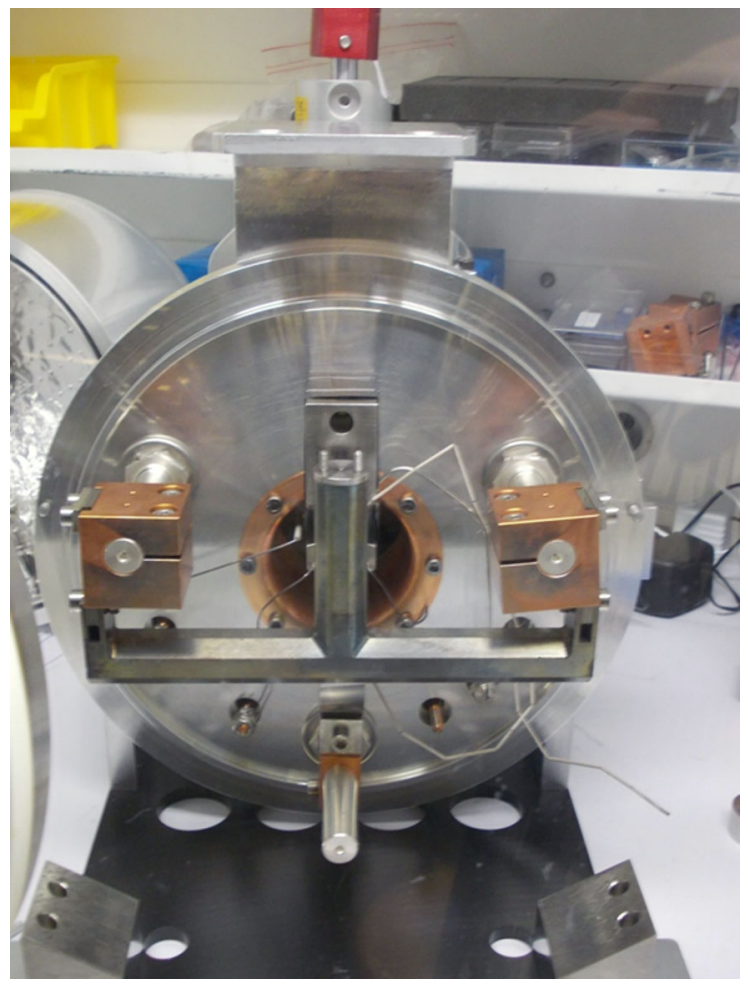

FIG. 6. Picture of the molten salt static target unit used at ISOLDE. 


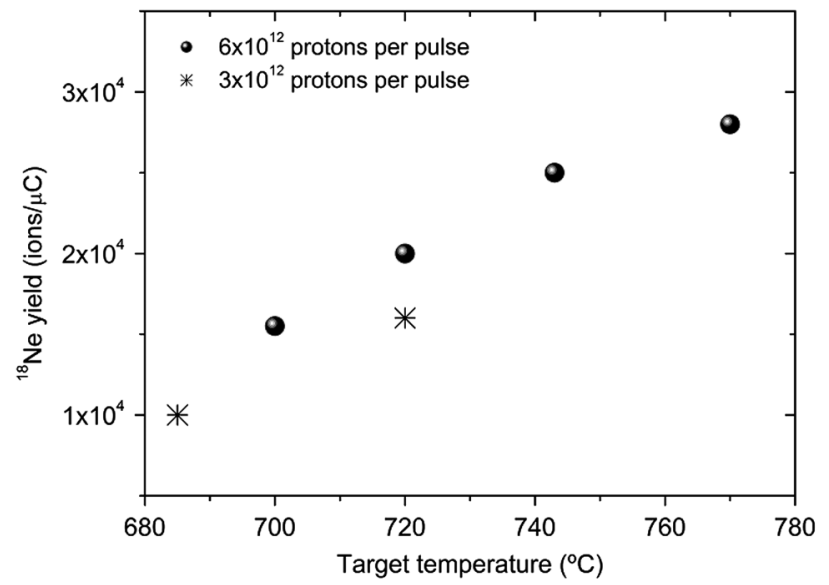

FIG. 7. Experimentally measured yields for different target temperatures and different proton beam intensities.

the PSB accelerator. The extraction efficiency and deduced yields have been studied as a function of the target temperature and proton beam intensities. The target unit has been kept above its melting point during the experimental run.

Figure 7 shows representative data of the production yields of ${ }^{18} \mathrm{Ne}$ at different target temperatures and proton beam intensities. The efficiencies required to calculate the atom production from a measured beam intensity were determined previously with stable tracers. From the measured yields at different target temperatures, the ${ }^{18} \mathrm{Ne}$ production varied from $1 \times 10^{4}$ to $3.3 \times 10^{4}{ }^{18} \mathrm{Ne} / \mu \mathrm{C}$ of incident protons. The present results validate the use of NaF-based salts in the production of ${ }^{18} \mathrm{Ne}$ as well as its use in a molten salt loop target. The circulating molten salt target will improve the diffusion time of ${ }^{18} \mathrm{Ne}$ and rates of $1 \times 10^{13}$ ${ }^{18} \mathrm{Ne} / \mathrm{s}$ are expected for $160 \mathrm{MeV}, 7 \mathrm{~mA}$ proton beam.

\section{PRODUCTION OF THE HIGH-Q ISOTOPES}

\section{A. The production ring}

To produce the ion pair ${ }^{8} \mathrm{Li} /{ }^{8} \mathrm{~B}$ [13] requires a compact synchrotron in which a $25 \mathrm{MeV}$ lithium ion beam circulates and interacts with a $\mathrm{D}$ or ${ }^{3} \mathrm{He}$ supersonic gas-jet target, to exploit the ${ }^{7} \mathrm{Li}(\mathrm{d}, \mathrm{p}){ }^{8} \mathrm{Li}$ and ${ }^{6} \mathrm{Li}(3 \mathrm{He}, \mathrm{n}){ }^{8} \mathrm{~B}$ reaction channels. The radioactive isotopes, produced at every passage through the target, are collected by a special device which stops and transports them to the charge-breeder ECR source by a diffusion/effusion ISOL-like mechanism, for further acceleration through the beta beam complex.

The choice of reverse kinematics (projectile heavier than target) has the advantage that most of the primary beam can be recycled, the ${ }^{8} \mathrm{Li}$ being produced in a narrow cone in the forward hemisphere with a velocity close to the beam velocity. However, since the angular spread of for example ${ }^{8} \mathrm{Li}$ is confined in a cone of only $11^{\circ}$, the amount of produced ions implanted for further reacceleration in a properly positioned absorber will strongly depend on the angular distribution of the reaction products. Consequently, one requirement of the system above is the knowledge of the angular distribution of the produced isotopes.

The stored beam is expected to survive for several thousands of turns, corresponding to the production characteristic time for the target thickness proposed in [13] and according to this scheme, the ionization cooling [25,26], provided by the target itself and a suitable rf system would be sufficient to compensate for multiple Coulomb scattering and energy straggling. First the ionization cooling mechanism is introduced, giving an estimation for the cooling potential for a beta beam production ring. The lattice design, [27], the ring parameters are then reported. Finally, the tracking simulations work [28] and the results in terms of emittance evolution and beam losses are presented. In the second part, technological solutions and challenges for the production ring are discussed, with special attention to the requirements for the gas-jet target, the stable Li source, the rf cavity and the vacuum issues. Finally, when the feasibility of the proposal cannot be easily demonstrated and/or when we think it could be an interesting option to be considered, alternative solutions are identified and discussed.

\section{The accelerator}

In order to produce ${ }^{8} \mathrm{Li}$ and ${ }^{8} \mathrm{~B}$ from the reactions ${ }^{7} \mathrm{Li}(\mathrm{d}, \mathrm{p}){ }^{8} \mathrm{Li}$ and ${ }^{6} \mathrm{Li}(3 \mathrm{He}, \mathrm{n}){ }^{8} \mathrm{~B}$, [13] it is proposed to use a compact ring in which a lithium beam is stored and interacts with a $\mathrm{D}$ or ${ }^{3} \mathrm{He}$ supersonic gas-jet target. The small synchrotron has a circumference of about $10 \mathrm{~m}$ and the kinetic energy of the incoming beam is $25 \mathrm{MeV}$, giving a relativistic beta of about $\beta_{r} \sim 0.1$. The ions are injected as $\mathrm{Li}^{+1}$ at the target location via a charge-exchange method where the target itself is acting as a charge stripper. At $25 \mathrm{MeV}$, the circulating beam is fully stripped. The radioactive isotopes, produced at every passage through the target, are emitted in a narrow angular cone of about $8^{\circ}$ (Sec. III D). A special collection device (Sec. III B) stops them and transports them to the charge-breeder ECR source, for further acceleration through the beta beam complex. Due to the interaction with the target, the stored beam suffers longitudinal and transverse emittance blowup, induced by multiple Coulomb scattering and energy straggling. The beam degradation is kept under control with the ionization cooling mechanism provided by the target itself and a suitable rf system.

\section{The circulating beam}

The number of particles $N$ circulating in the ring is given by

$$
\frac{d N}{d t}=-\frac{1}{\tau} N+I_{\text {source }}
$$


Following [13], in order to produce $10^{14}$ radioactive isotopes per second, the ${ }^{7} \mathrm{Li}$ ion source has to provide $I_{\text {source }}=160 \mu \mathrm{A}=10^{15}$ ions/s. Being $\tau=10^{4}$ turns/ $f_{\text {rev }}=3 \mathrm{~ms}$, the nuclear lifetime, after a few ms transient, there will be some $10^{12}{ }^{7} \mathrm{Li}$ particles circulating in the ring. For the ${ }^{8} \mathrm{~B}$ production, since the nuclear cross section is a factor 10 smaller, these quantities have to be increased by the same factor.

\section{The internal target}

The circulating Li beam is interacting with the production target at every passage in the ring. According to [13] for the energies of interest, the cross section for the nuclear reaction ${ }^{7} \mathrm{Li}(\mathrm{d}, \mathrm{p})^{8} \mathrm{Li}$ is about $100 \mathrm{mbarn}$, while for the ${ }^{6} \mathrm{Li}(3 \mathrm{He}, \mathrm{n})^{8} \mathrm{~B}$ reaction it is about 10 mbarn (see also $[29,30])$.

The total cross section, the sum of the nuclear elastic and inelastic reaction cross sections, which causes the ejection of the particle from the beam, is typically of 1 barn for both ${ }^{6} \mathrm{Li}$ and ${ }^{7} \mathrm{Li}$ nuclei and, assuming a target thickness $t=$ $0.277 \mathrm{mg} / \mathrm{cm}^{2}$ [13], this corresponds to a nuclear beam lifetime of about $\mathrm{n}=104$ turns.

The blowup due to multiple Coulomb scattering is evaluated using the Moliere rms angle equation:

$$
\Theta_{c}=\sqrt{\left\langle\Theta^{2}\right\rangle}=\frac{14.1 \mathrm{MeV}}{\beta_{r} c p} z \sqrt{\frac{t}{\chi_{0}}}\left[1+0.038 \ln \frac{t}{\chi_{0}}\right],
$$

where $\beta_{r} c, \gamma_{r}, p$ and $z$ are the velocity, relativistic mass factor, momentum and charge of the incident ion and $\chi_{0}$ is the radiation length.

The mean energy lost at the target is estimated via the Bethe-Bloch formula [31]:

$$
\begin{gathered}
\Delta E_{B B}=\left\langle\frac{d E_{L}}{d x}\right\rangle t \Rightarrow \\
\Delta E_{B B}=K z^{2} \frac{Z}{A} \frac{1}{\beta_{r}^{2}}\left[\frac{1}{2} \ln \frac{2 m_{e} c^{2} \beta_{r}^{2} \gamma_{r}^{2} T^{m}}{I^{2}}-\beta_{r}^{2}-\frac{1}{2} \delta\left(\beta_{r}^{2} \gamma_{r}^{2}\right)\right] t,
\end{gathered}
$$

where $A, Z$ and $I$ are the target atomic mass, charge and mean excitation energy. The quantity

$$
T_{m}=\frac{2 m_{e} c^{2} \beta_{r}^{2} \gamma_{r}^{2}}{1+2 \gamma_{r} m_{e} / M+\left(m_{e} / M\right)^{2}}
$$

is the maximum kinetic energy which can be imparted to a free electron in a single collision, with $m_{e}$ the electron mass and $M$ the mass of the incident particle, and

$$
K=4 \pi N_{A} r_{e}^{2} m_{e} c^{2}
$$

is a constant, $r_{e}$ the classical electron radius and $N_{A}$ Avogadro's number.
For a target thickness of $t=0.277 \mathrm{mg} / \mathrm{cm}^{2}$ [13], the average energy lost by a lithium ion is $300 \mathrm{keV}$, a value that needs to be restored by a strong if system.

Energy fluctuations are assumed to have a Gaussian distribution, with a root-mean square (rms) width of about $\sqrt{\left\langle\delta_{r m s}^{2}\right\rangle}=15 \mathrm{keV}$, as from Table 1 in [13]. Losses due to single large-scattering events [32] and by intrabeam scattering are for the time being not included in the computations.

\section{Ionization cooling}

The ionization cooling [25] is recently receiving large attention for the fast cooling of muons for a Neutrino Factory or a Muon Collider [26]. It is based on the principle that a beam traversing a material looses energy and only its longitudinal component is recovered in the rf cavities, with the net effect of a transverse emittance shrinking.

In analogy to synchrotron radiation damping, one can introduce [26] partition numbers, whose sum is invariant, to characterize the cooling rates in the three planes and define equilibrium emittances from the balance between the cooling terms and the heating ones.

The challenge of applying ionization cooling for lowenergy ions resides in the strongly negative slope of the Bethe-Bloch formula [31] for the energies of interest. In particular, $\left(\delta E_{\text {loss }} / \delta p\right)<0$ means that for an increase of particle momentum, the energy losses in the material becomes weaker, thus causing strong heating, instead of cooling, in the longitudinal plane. Longitudinal cooling can be achieved by introducing coupling with the horizontal plane via the dispersion and by using a wedge-shaped absorber in a dispersive region, but since the sum of the partition numbers is a constant (and in this case only slightly positive [33]), one can achieve longitudinal cooling only at expenses of the transverse one. Following the derivation of [26], and using parameters from [13,27] for the production ring we find that [28] we have to introduce coupling in the transverse plane, in the region of the target, to achieve cooling on the longitudinal plane. This can be done by introducing a wedge-shaped target and using the dispersion in the target area. However the total cooling power, which is the sum of the partition numbers in the three planes, cannot be changed.

The result of the analysis shows that for the case of the production ring for beta beam isotopes, using a ${ }^{7} \mathrm{Li}$ or ${ }^{6} \mathrm{Li}$ beam at $25 \mathrm{MeV}$ impinging on a $\mathrm{D}$ or ${ }^{3} \mathrm{He}$ target, the cooling efficiency is very low, almost zero. This depends on the slope of the Bethe-Bloch equation and could be improved only by changing the beam energy, on which there is not much freedom since it is set to optimize the production cross section. The practical meaning, for the production ring application, is that there is a very small margin for cooling and only in the case of perfect emittance exchange, achieved by coupling the longitudinal plane both with the horizontal and with the vertical, it will be possible 


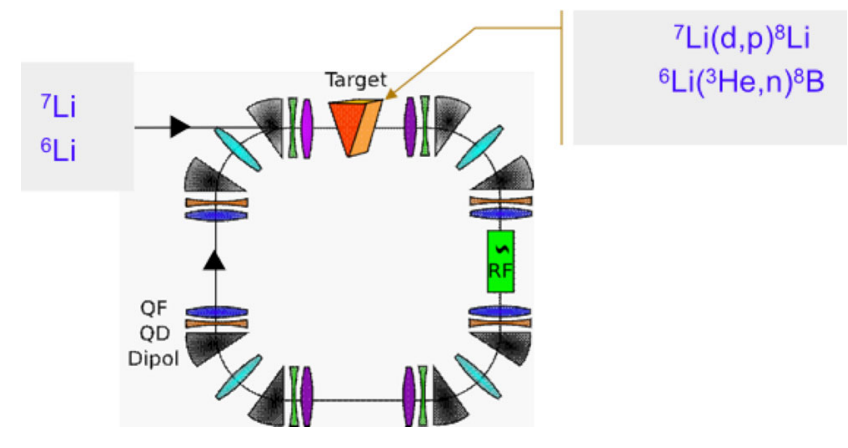

FIG. 8. The production ring.

to keep the beam size under control, as already pointed out in [33].

\section{The proposed lattice of the production ring}

The optics of the $12 \mathrm{~m}$ long production ring for the $25 \mathrm{MeV}{ }^{7} \mathrm{Li}$ ions (to produce ${ }^{8} \mathrm{Li}$ isotopes) is shown in Fig. 8 and the design is well documented in [27].

The ring has a twofold symmetry: two of the straight sections have zero dispersion, in order to accommodate the rf cavity(ies), the other two, instead, have an horizontal dispersion of $50 \mathrm{~cm}$, as required by the specifications for the production target, which will be installed in one of them. Table II summarizes the ring parameters.

For the simulations, the working point of $(2.58,1.63)$ has been chosen. The horizontal $\beta_{x}$ is for the moment of about $2.6 \mathrm{~m}$ at the target and leads to important beam blowup due to multiple Coulomb scattering.

For particles with "large" momentum offset (i.e., of the order of $1 \%$ ), the large negative chromaticity may induce resonance crossing and losses. A first attempt to include sextupoles in the lattice to compensate the chromaticity led to dynamic aperture problems. A trade-off between the increase in tune spread and the reduction in dynamic aperture has to be found. Moreover, a large second order

TABLE II. Beta-beam requirements for the production ring.

\begin{tabular}{lcc}
\hline \hline Parameter & Label & Value \\
\hline Particle & & ${ }^{7} \mathrm{Li}$ \\
Kinetic energy & $E_{B B}$ & $300 \mathrm{kEV}$ \\
Relativistic mass factor & $\gamma_{r}$ & 1.00383 \\
Beam rigidity & $B \rho$ & $0.636 \mathrm{Tm}$ \\
Circumference & $\mathrm{C}$ & $12 \mathrm{~m}$ \\
Revolution frequency & $f_{\text {rev }}$ & $2.18 \mathrm{MHz}$ \\
Transition $\gamma$ & $\gamma_{t}$ & 3.58 \\
Tune & $Q_{x, y}$ & $2.58,1.63$ \\
Natural chromaticity & $Q_{x, y}^{,}$ & $-3.67,-3.58$ \\
$\beta$ @ target & $\beta_{x, y}^{*}$ & $2.62 \mathrm{~m}, 0.35 \mathrm{~m}$ \\
Dispersion @ target & $\mathrm{D}_{x, y}^{*}$ & $0.523 \mathrm{~m}, 0 \mathrm{~m}$ \\
Target thickness & $\mathrm{t}_{0}$ & $0.27 \mathrm{mg} / \mathrm{cm}^{2}$ \\
Target thickness & $\mathrm{n}_{t}$ & $10^{19} \mathrm{atoms} / \mathrm{cm}^{2}$ \\
Energy loss @ target & $\mathrm{E}_{c}$ & $25 \mathrm{MeV}$ \\
\hline \hline
\end{tabular}

dispersion in the straight sections leads to a nonzero dispersion in the rf cavity for particles with a $1 \%$ momentum offset and to a $10 \%$ difference in the cooling section, which may need to be taken into account. This lattice, which still needs to be tuned for optimizing the cooling efficiency, is used to set up tracking simulations and for identifying the parameters to reduce the blowup [34].

\section{The code SIXTRACK for the production ring}

SiXTRACK [19] is a fully 6D, single-particle tracking code, based on high order truncation of Taylor expansion, which is widely used at CERN for dynamic aperture studies and for collimation. The code had to be adapted for the production ring simulations. The production target has been implemented in the code as a special element and the interaction with matter modeled by simple analytical formulas. Since SixTrack can only deal with protons, an equivalent proton beam is tracked, with the same rigidity $(\mathrm{B} \rho)$ and the same momentum $\Delta p_{r f} / p$ recovered at the rf cavity. Before the interaction with the target, the proton energy is converted to the ${ }^{7} \mathrm{Li}$ equivalent and then back again after the target [27]. The equivalent proton energy is $19 \mathrm{MeV}$ and the energy recovered at the rf cavity is $\Delta E_{r f}=$ $0.22 \mathrm{MeV}$ for the reference particle. The rf voltage and synchrotron phase, for a harmonic number $h=1$, have been set to $V=860.6 \mathrm{kV}$ and $\phi_{s}=15^{\circ}$, from considerations of bucket height, but this can be further tuned. Furthermore, a few beam diagnostics elements have been included in SiXTRACK, e.g., the possibility to have the turn by turn rms emittance evolution in the three planes.

Since SixTRACK is mainly used for LHC tracking and, since there is not much experience with low energy machines, it was necessary to perform a benchmark with MADX and PTC. Beta functions and dispersion for one half of the ring, for a momentum offset of $1 \%$ has been calculated and results have been compared. Even for this large momentum offset, both MADX and SIXTRACK, which are using a truncated Taylor expansion, are in very good agreement with PTC, which is using the exact Hamiltonian.

For a rectangular target some expected blowup in the momentum spread was found and indeed some cooling in the transverse plane up to about 300 turns. However, when the momentum spread goes above 2\% the transverse emittances increase considerably. The particles are lost in the dispersive regions. The emittance blowup in the transverse plane may have two explanations: the uncorrected chromaticity may cause resonance crossings and the large second order dispersion at the place where the emittance is computed generates an artificial emittance increase due to particles with nonzero dispersion whose invariant is not correctly evaluated at this stage. If the analysis is restricted to 300 turns the values found in the simulations are in agreement with the analytical estimations for the transverse equilibrium emittances of $\epsilon_{x}=87.7 \mathrm{~mm} \mathrm{mrad}$ and $\epsilon_{y}=11.7 \mathrm{~mm} \mathrm{mrad}$. 


\section{The choice of wedge angle}

A wedge-shaped target in a dispersive region is used to transfer the cooling from the horizontal to the longitudinal plane. By linearizing the Bethe-Bloch formula, with respect to the target thickness variation $\delta t$ and the particle energy offset $\delta E$, one obtains

$$
\left.E_{B B}\left(t, E_{c}\right) \approx \frac{d E}{d s}\right|_{E_{c 0}} t_{0}+\left.\frac{d E}{d s}\right|_{E_{c 0}} \Delta t+\left.\frac{\partial E}{\partial s}\right|_{E_{c 0}} t_{0} \Delta E_{c} .
$$

The first term is the mean energy lost by a beam of nominal energy $E_{c 0}$, traversing a target of uniform thickness $t_{0}$, and it is the energy recovered in the rf cavity by the synchronous particle. The second and third terms both depend on the particle momentum offset $(\delta \mathrm{p} / \mathrm{p})$ :

$$
\begin{gathered}
\Delta E_{c}=E_{c} \frac{\gamma_{r}+1}{\gamma_{r}} \frac{\Delta p}{p}, \\
\Delta t=2 \rho \tan \frac{w}{2} \Delta x=2 \rho \tan \frac{w}{2} \Delta x D^{*} \frac{\Delta p}{p},
\end{gathered}
$$

where $\rho$ is the target density, $w$ is the angle of the wedge and $\Delta x$ is the horizontal offset, induced by the dispersion $D^{*}$ at the target. By playing with the dispersion and the wedge angle it is possible to compensate for the difference in mean loss value due to different particle energy and, in particular, to fully compensate for the losses dependence on the momentum offset if

$$
D^{*} \tan \frac{w}{2}=\left.\frac{1}{2 \rho}\left(\frac{d E}{d s}\right)_{E_{c 0}}^{-1} \frac{\gamma_{r}+1}{\gamma_{r}} \frac{\partial}{\partial E_{c}}\left(\frac{d E}{d s}\right)\right|_{E_{c 0}} t_{0} E_{c} .
$$

From these considerations, the angle necessary to keep a constant momentum spread, thus to have no blowup in the longitudinal plane, is $w=15^{\circ}$, but, if one would choose this value, the blowup in the horizontal plane would be too large and would lead to losses comparable to the zerowedge case. Indeed, a $w=6^{\circ}$ angle is the best compromise between the blowup in the horizontal and longitudinal planes (see [35] for more details).

For a $w=6^{\circ}$ angle, the momentum spread increase is smaller than in the case of a rectangular target $\left(w=0^{\circ}\right)$, but this is obtained at the expense of a more important horizontal blowup. In the vertical plane the cooling is the same as before, since there is no coupling. Increasing the wedge angle to as high as $12^{\circ}$ leads again to strong losses, due to the uncontrolled horizontal blowup. Even for the best case $\left(w=6^{\circ}\right)$, after 900 turns $60 \%$ of the beam is lost in the machine. This has to be compared to the expected production rate, which generates a decrease of the circulating beam with a characteristic time of $~ 104$ turns. These results can be improved by minimizing the horizontal beta function value at the target position and by introducing coupling with the vertical dimension, to share the cooling power in the three planes.

\section{The primary ions source challenges}

According to [13], for the energies of interest for the ${ }^{6} \mathrm{Li}$ and ${ }^{7} \mathrm{Li}$ nuclei, the total cross section is of the order of 1 barn. For the nuclear reaction ${ }^{7} \mathrm{Li}(\mathrm{d}, \mathrm{p})^{8} \mathrm{Li}$, the cross section is about 100 mbarn at $25 \mathrm{MeV}$, see III D, meaning that $10 \%$ of the interacting particles will produce a useful isotope. Therefore, in order to reach the $10^{14} / \mathrm{s}$ radioactive isotope flux from the production ring, as required from physics, one would need $10^{15} / \mathrm{s}{ }^{7} \mathrm{Li}$ particles injected. Assuming $100 \%$ transmission efficiency in the linac, this corresponds to $160 \mu \mathrm{A}$ from the ${ }^{7} \mathrm{Li}$ source. Existing ECRIS only reach some $\sim 30 \mu \mathrm{A}$.

The primary ion intensity is not considered to be a show stopper for the ${ }^{8} \mathrm{Li}$ production, since several sources could be added in parallel to feed the linac, and/or R\&D has to be pushed.

For the ${ }^{6} \mathrm{Li}\left({ }^{3} \mathrm{He}, \mathrm{n}\right){ }^{8} \mathrm{~B}$ reaction, the cross section is less (see Sec. III C) so more intensity should be provided from the source, which is challenging.

\section{The production ring rf cavity}

By traversing the $0.27 \mathrm{mg} / \mathrm{cm}^{2}$ thick internal target, the lithium ions will lose about $300 \mathrm{keV}$ [13]. This energy has to be restored by an $\mathrm{rf}$ cavity. Since the revolution frequency is $\sim 3 \mathrm{MHz}$, and the harmonic number should be as small as possible, a low-frequency cavity is needed. Moreover, the cavity should be as compact as possible, because of the space constraints. The solution is to use an evacuated cavity with capacitive loading, in order to keep the size below $2 \mathrm{~m}$. A typical example at CERN is the bunch rotation cavity for Antiproton Collector (now used in the $\mathrm{AD}$ ) which reaches $750 \mathrm{kV}$ at $9.55 \mathrm{MHz}$ [36]. It is a pulsed device dissipating $660 \mathrm{~kW}$ at full voltage. At $300 \mathrm{kV}$, operation in $\mathrm{cw}$ would be feasible.

\section{Charge exchange injection}

Particles are injected in the ring as $\mathrm{Li}^{1+}$ ions at the gas-jet target location, which will also act as a stripper, and the circulating ions will be fully stripped. The transfer line and the injection have to be designed, however the design will be simpler than for standard $\mathrm{H}^{-}$injection systems, as the stripper will stay in the circulating beam being the target itself.

\section{Beam scraper}

In order to clean out large amplitude particles and have losses concentrated in one location, a beam scraper can be envisaged e.g., in the dispersive region opposite to the target. 


\section{Target}

In order to produce a sufficient number of beta emitters per second, the gas-jet target density should be extremely high. In [35] is shown that today existing gas-jet and cluster-jet target reach a maximum of $10^{15}$ atoms $/ \mathrm{cm}^{2}$, which is 4 orders of magnitude less than the thickness proposed in [13] and that the needed gas flows would be a problem for the vacuum in the production ring.

\section{Discussion of possible solutions}

The required $10^{19}$ atoms $/ \mathrm{cm}^{2}$ thick gas-jet target in the accelerator vacuum environment represents the most crucial issue for the feasibility of the production ring. Possible solutions have been investigated: (i) Increasing the injected beam intensity, to reach the required ion production rate, is not feasible, since the proposed stable-ion sources are already at the limit of or beyond the available operational currents, (ii) Living with a poor vacuum in the machine, which could be a solution as long as the residual gas is "thin" with respect to the jet target, causes multipacting in the rf cavity and it is therefore not feasible. (iii) Separating the target by thin windows causes a significant additional emittance growth and extra rf power to compensate for energy losses. (iv) Working at different energies is not an option, since $25 \mathrm{MeV}$ is already the best compromise between good production cross section (which decreases with increasing energy) and stripping efficiency. (v) Running with a "conventional" gas-jet target, with a 4 orders of magnitude lower thickness, decreases the production rate by the same amount. This is partly compensated by the increase in lifetime which will also increase the circulating beam current. The space-charge limit is anyway at about $10^{12}$ ions/bunch therefore only a factor 10 can be gained. Moreover, since the energy lost and recovered in the rf cavity is smaller as well, the cooling rate is also lower by the same amount, therefore ionization cooling may not be efficient. (vi) Using already existing CERN rings, such as AD, ELENA or LEIR, deserves feasibility studies. They have a larger circumference which allows the storage of a higher number of ions, for the same space-charge constraints, and they are equipped with electron cooling, in case ionization cooling is weak. This solution is not as elegant as the one proposed in [13], but it may be considered if the production rates are high enough. (vii) Having a solid or liquid target allows to reach $10^{19}$ atoms $/ \mathrm{cm}^{2}$ target thickness. In this case it is preferable to have a lithium target and a deuterium or helium beam (direct kinematic). This is for the time being our preferred option and it is under study.

\section{Production ring conclusions}

We have analyzed in detail the proposal by [13] to use a compact ring with an internal target to produce ${ }^{8} \mathrm{Li}$ and ${ }^{8} \mathrm{~B}$ isotopes for the beta beams. A preliminary ring design is available. The optics studies have been done for the ${ }^{7} \mathrm{Li}(\mathrm{d}, \mathrm{p}){ }^{8} \mathrm{Li}$ inverse kinematics case, but they can be easily scaled to the other reactions. Due to the strongly negative slope of the Bethe-Bloch function at the energies of interest for the isotopes production, the total budget of ionization cooling that can be achieved is very low, almost zero, therefore one should not expect significant emittance reduction but, in the best case, only control of the beam blowup. 6D tracking tools are fully in place and predict what is expected from analytical ionization-cooling considerations. SixTrack code allows us to see also the high order effects, e.g., chromaticity and second order dispersion, therefore the blowup that is seen in the simulations is explained and could be corrected, although it is not so straightforward due to the small periodicity of the machine. The lattice requires careful tuning to maximize ionization-cooling efficiency and in particular the beta function at the target position needs to be reduced as much as possible. Coupling with the vertical plane should be introduced as well. Feasibility studies identified as a major issue the large thickness $\left(10^{19}\right.$ atoms $\left./ \mathrm{cm}^{2}\right)$ required for the gas-jet target in a vacuum environment. The direct kinematics approach looks more feasible from the point of view of the target density, although the thin liquid-films technology (used as heavy-ion strippers and as targets in nuclear physics) is still in early $R \& D$.

\section{B. The collection device 1. ${ }^{2} \mathrm{H}\left({ }^{7} \mathrm{Li},{ }^{8} \mathrm{Li}\right) p$. validation for the ${ }^{8} \mathrm{Li}$}

The collection device was designed and constructed for the experiments on the collection of ${ }^{8} \mathrm{~B}$ and ${ }^{8} \mathrm{Li}$ isotopes after production in a target in view of the use of the production ring. The first experiment was performed with ${ }^{7} \mathrm{Li} 2^{+}$beam provided by the isochronous cyclotron of the Centre de Recherches du Cyclotron at Louvain-la-Neuve. The R\&D work was organized in two phases: first, the design and construction of a collection device which will be validated with ${ }^{8} \mathrm{Li}$, then the experimental study of the possible ways to extract ${ }^{8} \mathrm{~B}$ from the collection device.

The inverse kinematics scheme was used. The ${ }^{7} \mathrm{Li}$ beam accelerated to $30 \mathrm{MeV}$ by the cyclotron is sent into a gas cell filled with $\mathrm{D}_{2}$ at about 150 mbar. After the energy losses in the foils in front of the gas the energy of the ${ }^{7} \mathrm{Li}$ beam is $24.9 \mathrm{MeV}$. The $\mathrm{D}_{2}$ gas target is made of a copper cylindrical cell, $17 \mathrm{~mm}$ in diameter and $15 \mathrm{~mm}$ in length, closed by two tantalum windows $5 \mu \mathrm{m}$ thick. The beam current is monitored by the scattering of the ${ }^{7} \mathrm{Li}$ beam on a gold foil installed just before the gas cell. The backscattered ions are detected by a silicon detector (PIPS detector $300 \mu \mathrm{m}$ thick) which is mounted at an angle of $166^{\circ}$. The typical beam intensity for the ${ }^{8} \mathrm{Li}$ runs is $10 \mathrm{pnA}$. In order to discriminate the production of the secondary particles, the beam was very well collimated with Ta collimators. The recoiling ${ }^{8} \mathrm{Li}$ are collected in a tantalum tube $(d=28 \mathrm{~mm}, l=112 \mathrm{~mm})$ in which they are slowed 


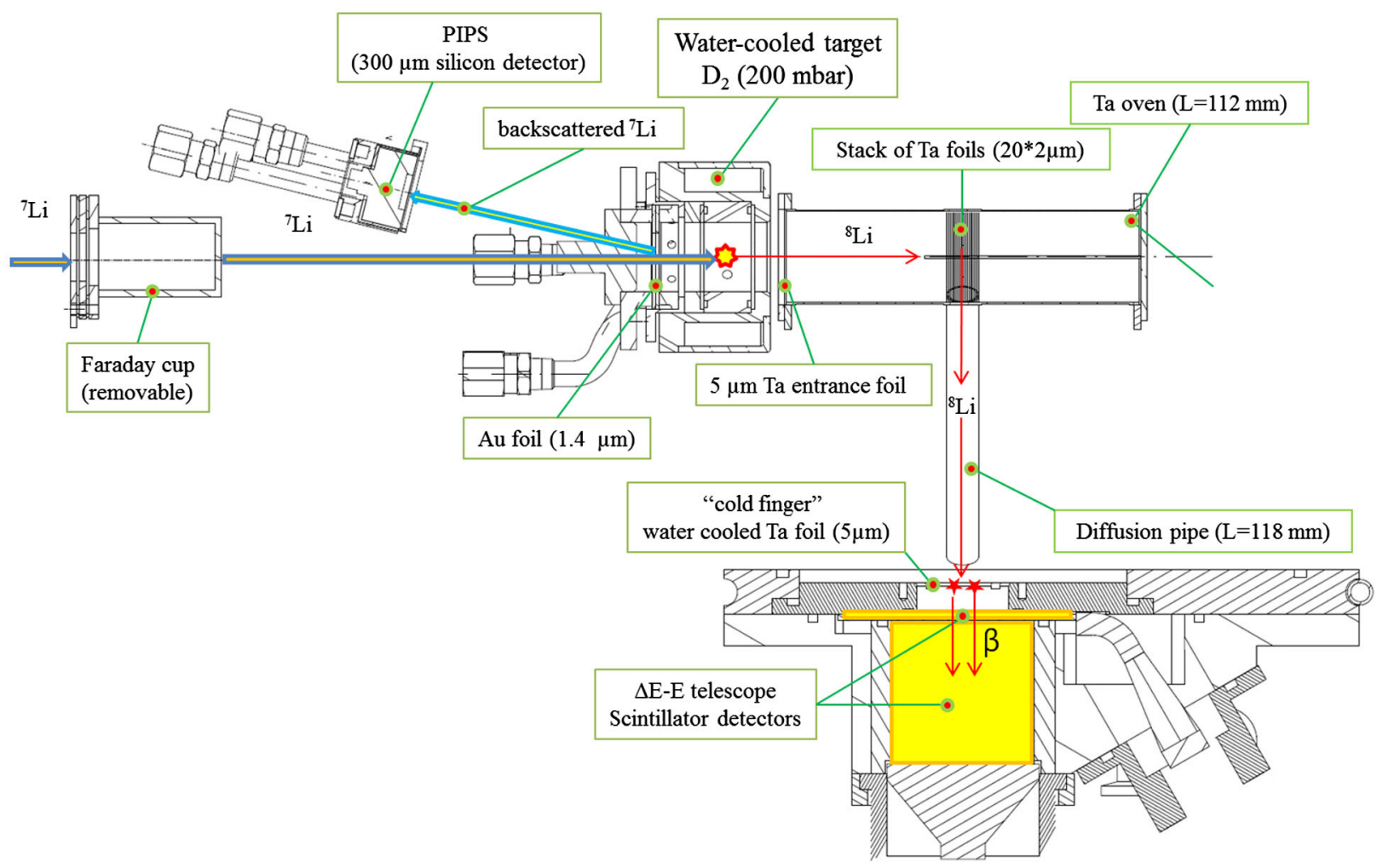

FIG. 9. Experimental setup for the ${ }^{8} \mathrm{Li}$ runs.

down by a set of $2 \mu \mathrm{m}$ thick tantalum foils. A diffusion pipe $(d=8 \mathrm{~mm}, l=118 \mathrm{~mm})$ bring the ${ }^{8} \mathrm{Li}$ atoms to a cold plate in front of a telescope made of 2 plastic scintillators to detect the beta decay (see Fig. 9). A set of power supplies allowed the heating of this collection device in order to favor the diffusion of the ${ }^{8} \mathrm{Li}$ ions.

The production of ${ }^{8} \mathrm{Li}$ is measured by detecting the $\beta^{-}$ associated with the ${ }^{8} \mathrm{Li} \beta$ decay (Fig. 10). In order to identify the nature of the secondary particles produced in the runs, the beam is pulsed and the betas are registered during the beam-off period only.

The time structure of the ${ }^{8} \mathrm{Li}$ experiments is given in Fig. 11. During a total cycle of $6 \mathrm{~s}$, the beam is on the target

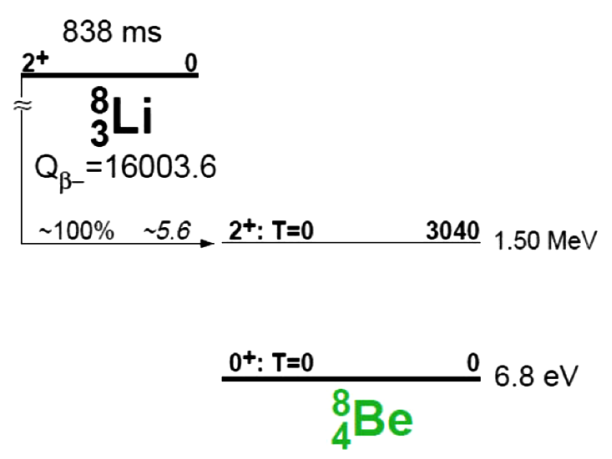

FIG. 10. Partial level scheme of the ${ }^{8} \mathrm{Li}$ decay. during $2 \mathrm{~s}$. After a time interval of $5 \mathrm{~ns}$, the measurement starts during $4 \mathrm{~s}$.

In parallel, a second setup (without collection device and oven) is used to measure the overall production of ${ }^{8} \mathrm{Li}$ and to check our detection system ("integral measurement"), see Fig. 12. The time structure is different; the beam is on the target during $2 \mathrm{~s}$ and afterwards the betas are detected during $8 \mathrm{~s}$.

To decrease the production of other products induced by the ${ }^{7} \mathrm{Li}$ beam we used degraders $(\mathrm{Cu}$ and $\mathrm{Al}$ foils, with respect of the Coulomb barrier value), see Fig. 12. To avoid

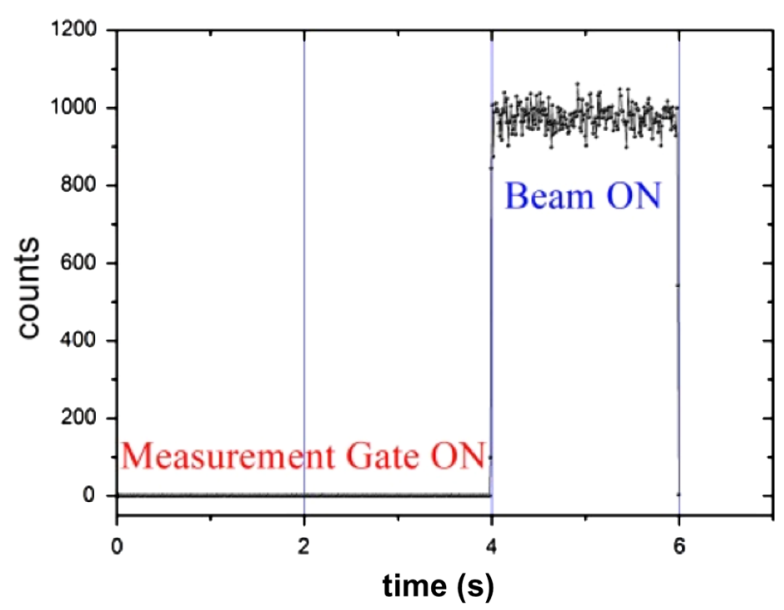

FIG. 11. Time structure used in the experiment. 


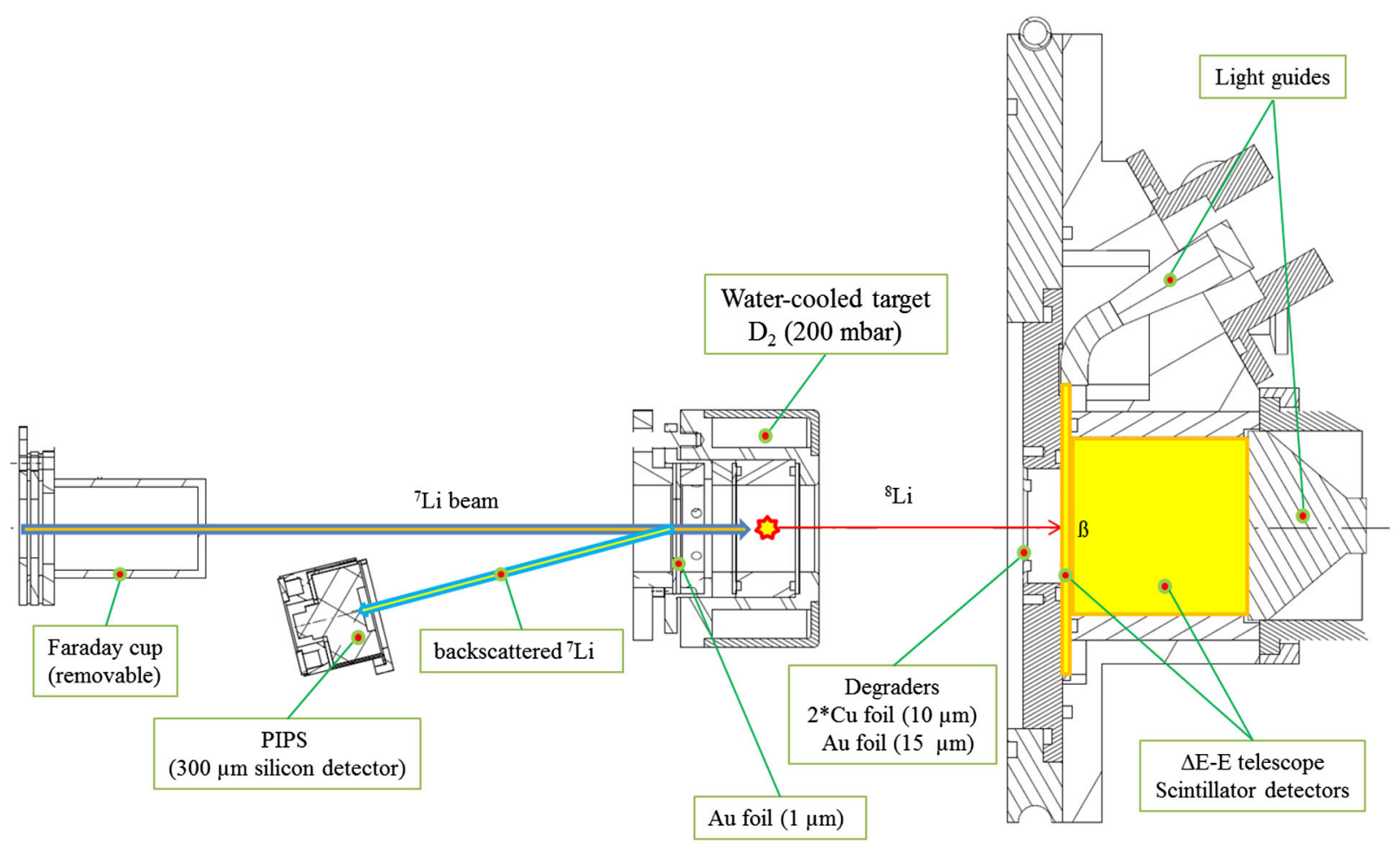

FIG. 12. Experimental setup without collection device.

any normalization factors and to keep the same geometry (dimensions, distances, angles) we used the same target unit in both cases. Figure 13 shows the decay curve of ${ }^{8} \mathrm{Li}$ obtained in the integral measurement. The obtained lifetime is very close to the value in the literature. The calculated efficiency of the detection system (the ratio between the amount of ${ }^{8} \mathrm{Li}$ we detect and the amount of ${ }^{8} \mathrm{Li}$ we produce in the target) is $27 \%$ with an uncertainty of $2 \%$. This value can be explained by the geometry of our setup: we stop the ${ }^{8} \mathrm{Li}$ nuclei in foils in front of the scintillators telescope and this gives us a geometrical efficiency of around $31 \%$.

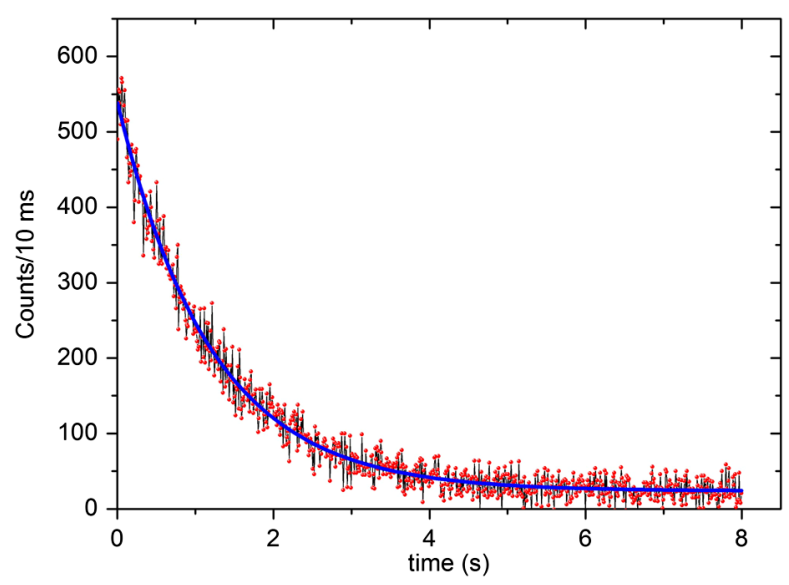

FIG. 13. Decay of ${ }^{8} \mathrm{Li}$ (setup without the collection device). $\chi^{2} / n=\mathrm{T}_{1 / 2}=826 \mathrm{~ms}$ in comparison with $\mathrm{T}_{1 / 2}=840 \mathrm{~ms}$ (from in literature adopted value).
The quantity of ${ }^{8} \mathrm{Li}$ produced during a run is calculated from the amount of backscattered beam, from the amplitude of the ${ }^{8} \mathrm{Li}$ exponential decay curve and from a factor which takes into account the time structure of the measurement. After the validation of our detection system we start measurements with the collection device with an oven. Usual decay curves at different temperatures are shown in Figs. 14(a) and 14(b).

\section{2. ${ }^{3} \mathrm{He}\left({ }^{6} \mathrm{Li},{ }^{8} \mathrm{~B}\right) n .{ }^{8} \mathrm{~B}$ experiments}

Knowing that the setup is working properly the ${ }^{8} \mathrm{~B}$ measurements is started. The ${ }^{8} \mathrm{~B}$ nuclei are produced by the reaction ${ }^{3} \mathrm{He}\left({ }^{6} \mathrm{Li},{ }^{8} \mathrm{~B}\right) \mathrm{n}$. The ${ }^{6} \mathrm{Li}$ beam accelerated to $32 \mathrm{MeV}$ by the cyclotron is sent into a gas cell filled with ${ }^{3} \mathrm{He}$ at about 200 mbar. After the energy losses in the foils in front of the gas the energy of the ${ }^{7} \mathrm{Li}$ beam is $29.3 \mathrm{MeV}$. Once again, in order to be able to compute an extraction efficiency, we first have to know the amount of produced ${ }^{8} \mathrm{~B}$ ions (integral measurement). The typical beam intensity for the ${ }^{6} \mathrm{Li}$ runs is 2 pnA (Fig. 15).

We want to try two completely different extraction schemes. In the first one, the ${ }^{8} \mathrm{~B}$ are slowed down in a stack of heated Ta foils and we want to see whether the ${ }^{8} \mathrm{~B}$ ions are able to escape as is the case with $\mathrm{Li}$ ions. In the second schema, the ${ }^{8} \mathrm{~B}$ are slowed down in $\mathrm{AlF}_{3}$ in which we could observe an exchange reaction $\mathrm{B}+\mathrm{AlF}_{3} \rightarrow \mathrm{Al}+$ $\mathrm{BF}_{3} \mathrm{BF}_{3}$ is a gas which can diffuse very easily up to the detection setup, if it is not dissociated by a too high temperature. 


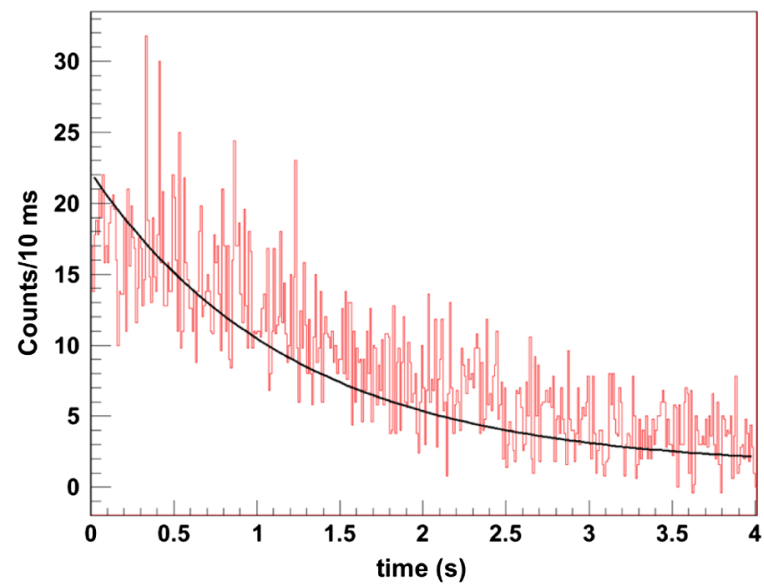

(a) $1450{ }^{\circ} \mathrm{C}$

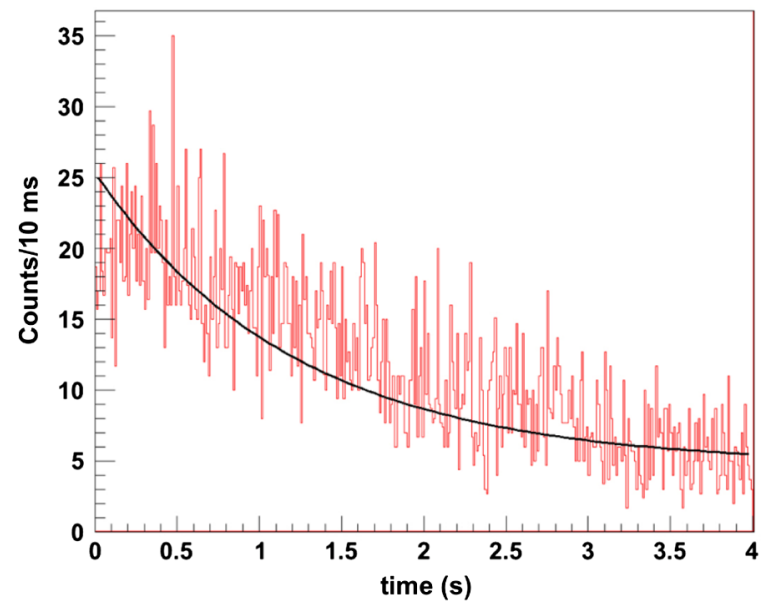

(b) $1650^{\circ} \mathrm{C}$

FIG. 14. Decay of ${ }^{8} \mathrm{Li}$ (setup with the collection device) at $14500^{\circ} \mathrm{C}$ and $1650^{\circ} \mathrm{C} \cdot \chi^{2} / n\left(1450^{\circ} \mathrm{C}\right)=574.7 / 397$; $\chi^{2} / n\left(1650^{\circ} \mathrm{C}\right)=494.4 / 398$.

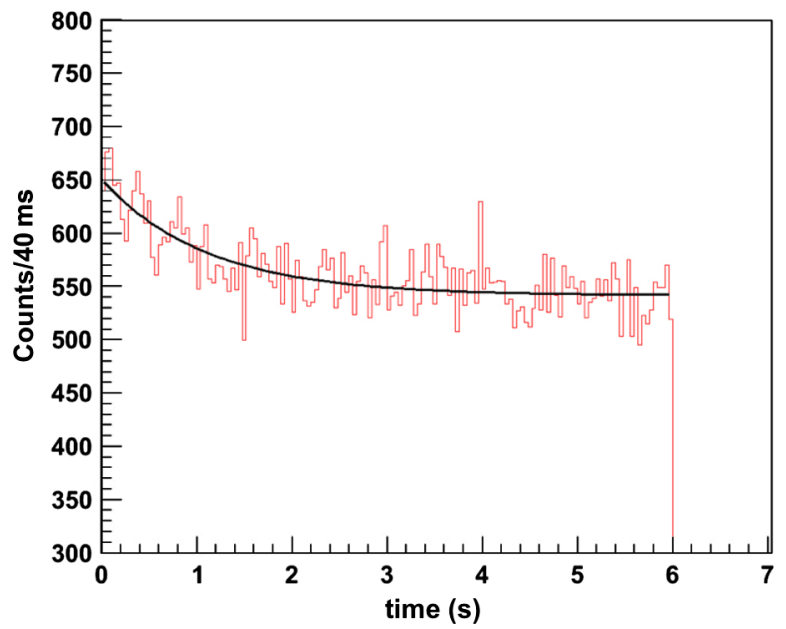

FIG. 15. Decay of ${ }^{8} \mathrm{~B}$ (setup without the collection device). $\chi^{2} / n=596.9 / 597 \mathrm{~T}_{1 / 2}=639 \mathrm{~ms}$ in comparison with $\mathrm{T}_{1 / 2}=$ $770 \mathrm{~ms}$ (from in literature adopted value).
In the first case (stack of Ta foils) the extraction and diffusion of the ${ }^{8} \mathrm{~B}$ ions is negligible and the decay curves are flat, consistent with a small random background. In the second case we fill the oven with $\mathrm{AlF}_{3}$ powder and heat the collection device to rise the effusion of $\mathrm{BF}_{3}$. The most representative plots are shown in Fig. 16.

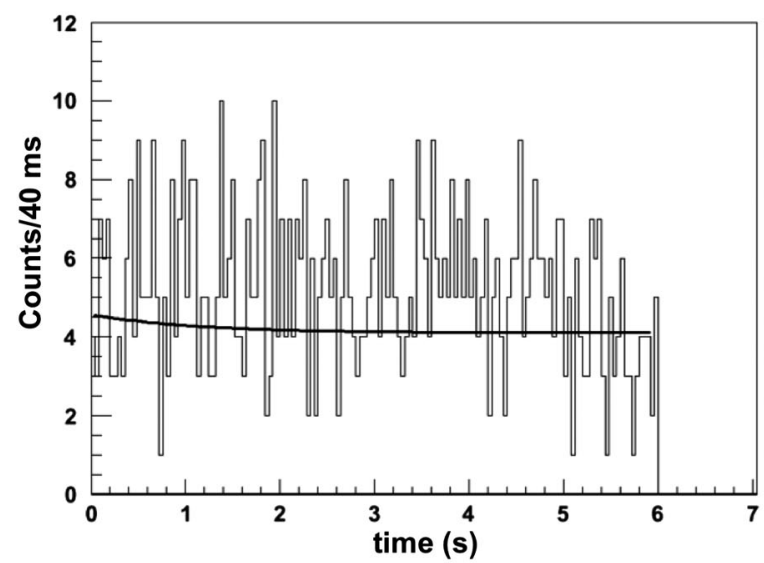

(a) $320^{\circ} \mathrm{C}$

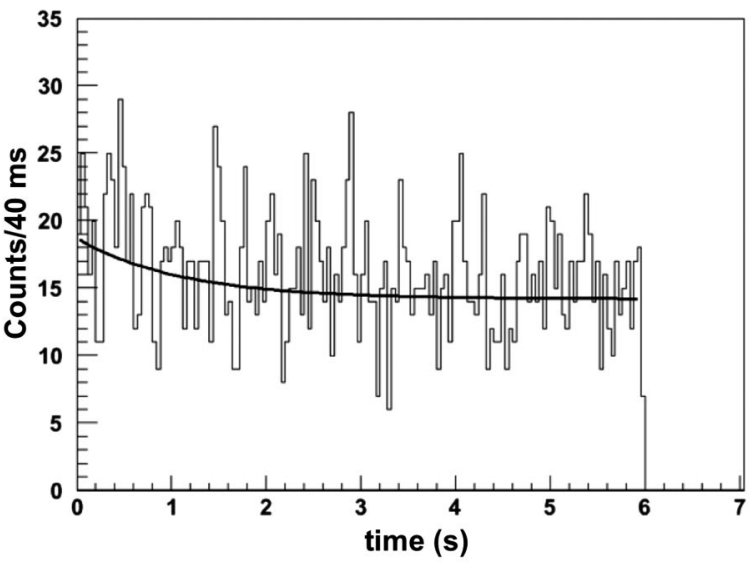

(b) $440{ }^{\circ} \mathrm{C}$

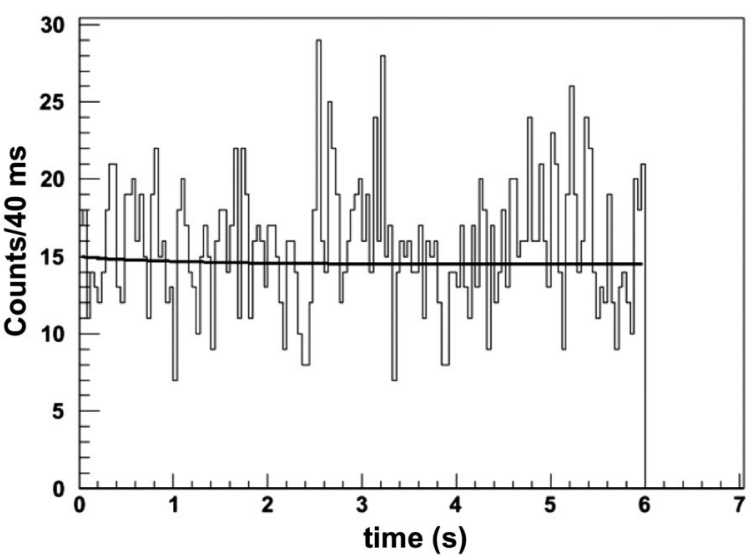

(c) $540{ }^{\circ} \mathrm{C}$

FIG. 16. Decay of 8B (setup with the collection device and $\left.\mathrm{AlF}_{3}\right)$ at $320^{\circ} \mathrm{C}(\mathrm{a}), 440{ }^{\circ} \mathrm{C}(\mathrm{b})$, and $540{ }^{\circ} \mathrm{C}(\mathrm{c})$. 
While the detected ${ }^{8} \mathrm{~B}$ amount is negligible at $320^{\circ} \mathrm{C}$ and $540{ }^{\circ} \mathrm{C},{ }^{8} \mathrm{~B}$ is obviously produced and extracted at $440{ }^{\circ} \mathrm{C}$. A possible explanation (which should be confirmed by additional measurements) is that at low temperatures the extraction efficiency is too low but at higher temperature the $\mathrm{BF}_{3}$ molecule is broken, giving an optimum at around $440{ }^{\circ} \mathrm{C}$. The observed extraction efficiency at $440{ }^{\circ} \mathrm{C}$ is $0.53 \pm 0.08 \%$. The most reasonable explanation of a so low efficiency is that a lot of $\mathrm{BF}_{3}$ is lost before it will reach the detection system: the tightness of the setup to deliver the $\mathrm{BF}_{3}$ up to a "cold finger" cooled with liquid nitrogen should be improved. This experiment is planned for around December 2012.

\section{C. ${ }^{8}$ B cross section measurements}

The EUROnu beta beam development needs measurements of the cross sections and angular distributions of the reaction products ${ }^{8} \mathrm{~B}$ and ${ }^{8} \mathrm{Li}$ from the reactions: (i) ${ }^{3} \mathrm{He}+{ }^{6} \mathrm{Li} \rightarrow{ }^{8} \mathrm{~B}+n$ (subject of this paper), (ii) ${ }^{7} \mathrm{Li}+\mathrm{d} \rightarrow{ }^{8} \mathrm{Li}+\mathrm{p}$ (see the paper of Vardaci et al. [37]).

The results of these measurements are necessary to design the accelerator and the other necessary equipment that will be used for the production of these isotopes, in particular to assess the performance of an internal target that also serves as a stripper and an absorber for ionization cooling of the circulating beam proposed by Rubbia et al. [13].

The total cross section of the ${ }^{8} \mathrm{~B}$ production in the ${ }^{6} \mathrm{Li}\left({ }^{3} \mathrm{He}, \mathrm{n}\right){ }^{8} \mathrm{~B}$ reaction was measured previously by using two different techniques. The results of the experiments using the measurement of the ${ }^{8} \mathrm{~B}$ positron decay reported in [30] and considered in the original proposal of Rubbia et al. [13] demonstrate the total cross section with at least a factor of 3 smaller with respect to the results from the experiment using the neutron time-of-flight method [38]. The results reported in [29] at the bombarding energies above $8 \mathrm{MeV}$ are not in agreement with the work of the other groups. Moreover, uncertainties of some experimental results are reaching $15 \%-20 \%$. Therefore, our aim was to accurately measure absolute cross section and the angular distribution of ${ }^{8} \mathrm{~B}$ produced in the ${ }^{6} \mathrm{Li}\left({ }^{3} \mathrm{He}, \mathrm{n}\right){ }^{8} \mathrm{~B}$ reaction by using the neutron-time-of-flight techniques employing the digital electronics, collecting high statistics and performing pulse-shape analysis (PSA).

\section{Experiment}

The experiment was done at the CN 7 MV Van De Graaff accelerator of Laboratori Nazionali di Legnaro.

The ${ }^{6} \mathrm{Li}\left({ }^{3} \mathrm{He}, \mathrm{n}\right){ }^{8} \mathrm{~B}$ reaction was studied using a $6.1 \mathrm{MeV}$ pulsed ${ }^{3} \mathrm{He}$ beam onto a $\mathrm{LiF} 500 \mu \mathrm{g} / \mathrm{cm}^{2}$ thick target. The $\mathrm{LiF}$ target was $95 \%$ enriched in ${ }^{6} \mathrm{Li}$ and evaporated on a $500 \mu \mathrm{g} / \mathrm{cm}^{2}$ thick Au backing. To minimize Li evaporation the target was cooled during the experiment and the gold backing was mounted towards the beam. The resulting beam energy after passing the $\mathrm{Au}$ and at the middle of the $\mathrm{LiF}$ target was $5.77 \mathrm{MeV}$. The pulsed beam structure was characterized by a $333 \mathrm{~ns}$ repetition rate and a $2 \mathrm{~ns}$ time resolution.

The emitted neutrons were measured via the time-offlight techniques by using eight large volume BC501 liquid scintillation detectors of the RIPEN modular array [39] upgraded with digital electronics. The detectors were placed at the distance of $2 \mathrm{~m}$ from the target at the angles of $15,23,31,39,50,80,110$ and 140 degrees. A $\Delta \mathrm{E}$ $(15 \mu \mathrm{m})-\mathrm{E}(200 \mu \mathrm{m})$ silicon telescope placed inside the scattering chamber at 150 degrees and at the distance of $56.5 \mathrm{~mm}$ from the target was used to continuously monitor the current intensity through the elastically scattered ${ }^{3} \mathrm{He}$ particles on gold. Possible contaminations have been taken into account and their evaluation has been considered through appropriate measurements. In particular measurements of ${ }^{3} \mathrm{He}$ on ${ }^{7} \mathrm{LiF},{ }^{12} \mathrm{C}$ have been performed. In addition, a measurement with no target has been performed for background determination.

The scintillator and silicon detector signals were recorded using two CAEN V1720 digitizers (12 bit, $250 \mathrm{Ms} / \mathrm{s}$ ) in the eight channels VME version communicating with a standard PC via a VME bridge (CAEN V1718). The software used for the data acquisition is a customized version of CAEN WAVEDUMP, able to handle and synchronize two or more digitizers. Three different kinds of information are expected to be obtained processing the scintillator signals: the energy release of the impinging particles, its time of flight and the pulse shape discrimination between neutrons and gammas. Data are processed using algorithms able to perform $\mathrm{RC} / \mathrm{CR}$ filters and constant fraction discriminator emulator. A proper baseline subtraction is computed from the raw data.

Energy calibration of the BC501 detectors was done using ${ }^{137} \mathrm{Cs},{ }^{60} \mathrm{Co}$ and ${ }^{88} \mathrm{Y}$ gamma sources. Silicon detectors calibration was performed using a triple $\mathrm{Am}-\mathrm{Pu}-\mathrm{Cm}$ alpha source.

The neutron gamma discrimination was achieved both by the time-of-flight and the zero-crossing method that rely on the longer tail of the neutron signals with respect to the gamma ones in liquid scintillators. Through the correlation between the zero crossing and the deposited energy of the interacting radiation two different loci relative to neutrons and gammas can be distinguished. A neutron detection threshold of about $150 \mathrm{keVee}(\mathrm{keV}$ electron equivalent) was achieved with the PSA discrimination. This threshold corresponds to a minimum neutron energy of about $0.5 \mathrm{MeV}$. The detection threshold determines the efficiency of the BC501 detectors that can be calculated by a Monte Carlo code as reported in Ref. [39]. The calculated intrinsic efficiency for the BC501 used in this work as a function of the neutron energy is reported in Fig. 17. From two-body kinematics calculations the energy range of the neutron coming from the ${ }^{6} \mathrm{Li}\left({ }^{3} \mathrm{He}, \mathrm{n}\right){ }^{8} \mathrm{~B}$ reaction at 


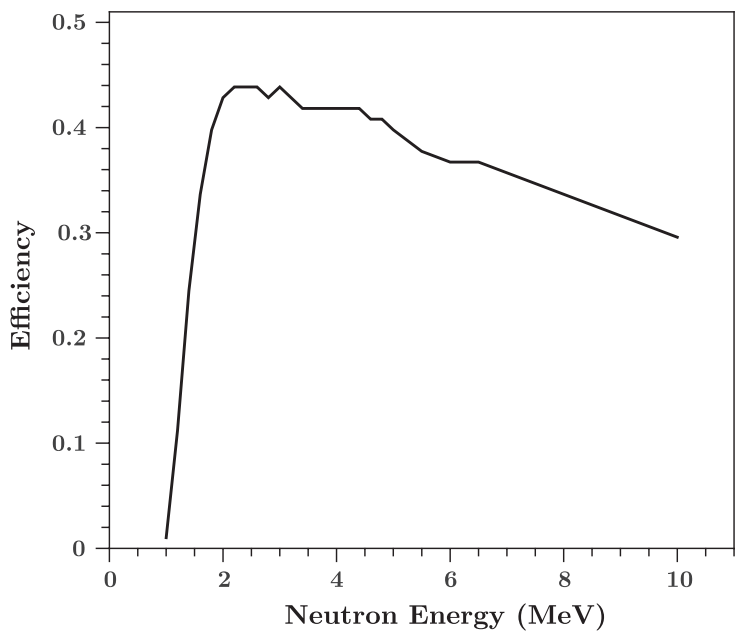

FIG. 17. Absolute neutron detection efficiency calculated for one RIPEN detector.

5.77 MeV is from $0.8 \mathrm{MeV}$ at the most backward angle to about $3 \mathrm{MeV}$ for the most forward detector.

In Fig. 18 the neutron time-of-flight spectrum of the scintillator detector positioned at 15 degrees is shown after the proper neutron signal selection from PSA. One can easily identify the two ${ }^{8} \mathrm{~B}$ peaks (ground state and the first excited state at $0.78 \mathrm{MeV}$ that immediately decay by proton emission) and the peak from the reaction ${ }^{12} \mathrm{C}\left({ }^{3} \mathrm{He}, \mathrm{n}\right){ }^{14} \mathrm{O}$ due to the carbon deposited on the target. The overall continuum is due to the three-body reaction ${ }^{6} \mathrm{Li}\left({ }^{3} \mathrm{He}, \mathrm{np}\right){ }^{7} \mathrm{Be}$. Uncorrelated background and continuum have been subtracted by using the sensitive nonlinear iterative peak clipping algorithm within the ROOT class TSpectrum ([40]).

From the area under the peaks of interest one can infer the differential cross section at the considered angles after correction for the detection efficiency and normalization to

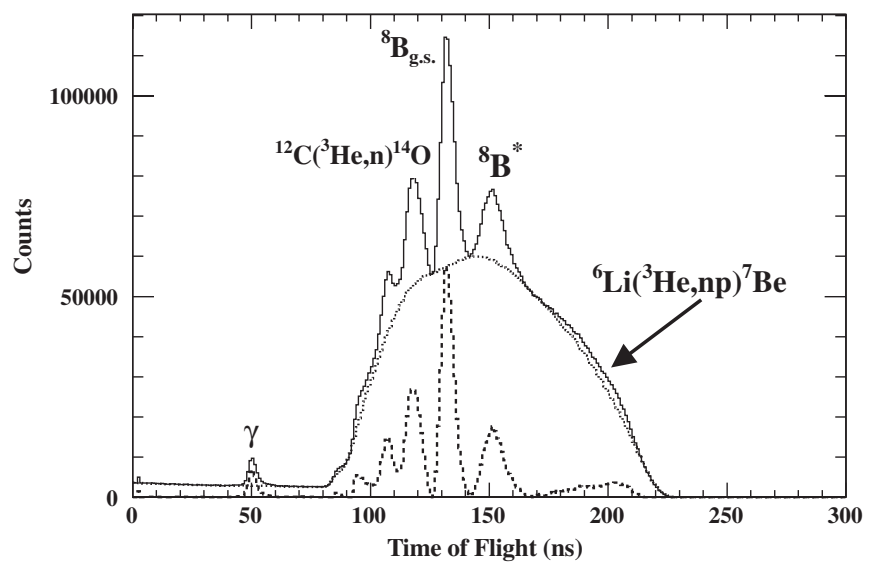

FIG. 18. Neutron time-of-flight spectrum at 15 degrees in the laboratory reference frame from the reaction ${ }^{6} \mathrm{Li}\left({ }^{3} \mathrm{He}, \mathrm{n}\right){ }^{8} \mathrm{~B}$ at $5.77 \mathrm{MeV}$. The time calibration is $1 \mathrm{~ns}$ per channel. The distance from the target to detector is $200 \mathrm{~cm}$. See text for details. the Rutherford scattering on the gold backing. The experimental angular distribution obtained is shown in Fig. 3 for the ${ }^{8} \mathrm{~B}$ ground state population. The error bars take into account all the uncertainties of the measure (solid angle of the detectors, target thickness, detection efficiency) and are of the order of $10 \%$.

\section{Results and discussion}

Theoretical calculations [41] were performed in order to compare the results of the absolute cross section and angular distribution obtained experimentally for the ground state of ${ }^{8} \mathrm{~B}$. The evaluations of the differential cross sections of the reaction ${ }^{6} \mathrm{Li}\left({ }^{3} \mathrm{He}, \mathrm{n}\right){ }^{8} \mathrm{~B}$ have been done by means of the "Zero Range Knock-out Distorted Wave Born Approximation" (ZR-KO-DWBA) [42] for twonucleon transfer with microscopic Bayman-Kallio form factors [43] using the code DWUCK4 [44].

The differential cross section for the allowed combination of transferred angular momenta $L S J$ ( $L$, orbital angular momentum transfer; $S$, spin transfer; $J$, total angular momentum transfer) is given by

$$
\begin{gathered}
\frac{d \sigma}{d \Omega}(\theta)=N\left(\sum_{L} A_{L}^{2} \frac{d \sigma_{L S J}}{d \Omega}(\theta)\right), \\
\frac{d \sigma_{L S J}}{d \Omega}(\theta)=10 \frac{m b}{f m^{2}} \frac{\left(2 I_{B}+1\right)}{\left(2 I_{A}+1\right)(2 J+1)} \sigma_{L S J}^{D W U C K 4}(\theta),
\end{gathered}
$$

where $I_{A}$ and $I_{B}$ are spins of the target and the product nuclei, respectively, $N$ and $A_{L}$ are renormalization factors that contain information about the unknown volume integrals and spectroscopic amplitudes of the corresponding configurations. The optical model parameters for entrance $\left({ }^{3} \mathrm{He}+{ }^{6} \mathrm{Li}\right)$ and exit $\left(\mathrm{n}+{ }^{8} \mathrm{~B}\right)$ channels extrapolated from corresponding global optical potentials $[45,46]$ were used. Single-particle wave functions for two-nucleon transfer form factor in DWUCK4 were calculated by the well-depth procedure with geometrical parameters $r_{0}=1.25 \mathrm{fm}$ and $a=0.65 \mathrm{fm}$. All volume integrals are equal to 1 . To estimate renormalization factors $N$ and $A_{L}^{2}$ the calculations for the case of beam energy $E=5.6 \mathrm{MeV}$ were performed and the results were compared to the experimental data of Ref. [38]. The resulting values used in our calculations were: $N=16679, A_{0}^{2}=0.878$ and $A_{2}^{2}=0.122$.

In Fig. 19 the experimental differential cross section in the center of mass frame is compared with the above discussed theoretical predictions. We found a reasonably good agreement between measurement and calculations at forward angles while the backward neutron emission is overestimated. The integrated measured cross section is $58 \pm 7 \mathrm{mb}$ to be compared with the $75 \mathrm{mb}$ calculated value.

We stress the fact that the present results are in very good agreement with the findings of earlier measurements using 


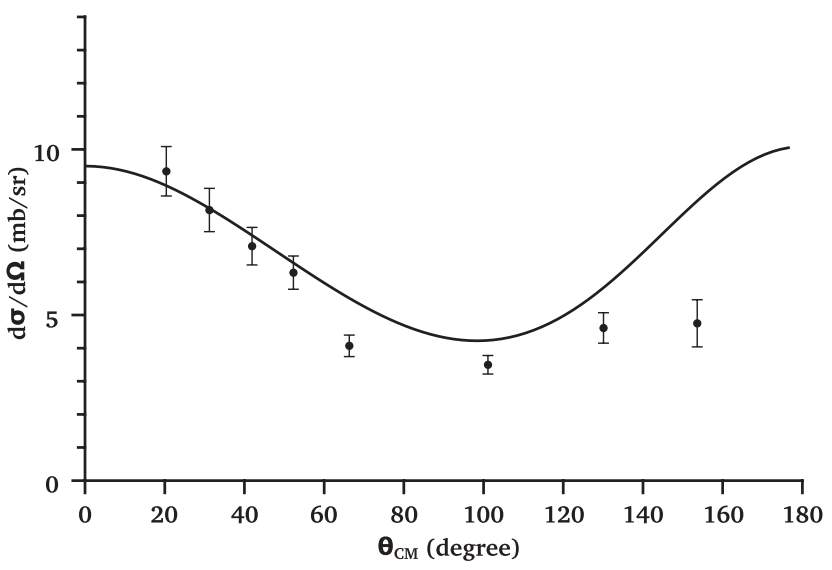

FIG. 19. Neutron angular distribution in the center of mass frame of the ${ }^{6} \mathrm{Li}\left({ }^{3} \mathrm{He}, \mathrm{n}\right)^{8} \mathrm{~B}$ reaction. The solid line is the result of theoretical predictions based on the DWUCK4 code.

the neutron time-of-flight method [38], thus confirming the disagreement with the positron counting results [29]. In Ref. [38] a deeper discussion can be found explaining the origin of the disagreement between experiment and calculations, mainly due to the reaction mechanisms involved (knock-out and/or nucleon transfer).

\section{Conclusions and outlook}

The angular distribution and cross section of the ${ }^{6} \mathrm{Li}\left({ }^{3} \mathrm{He}, \mathrm{n}\right){ }^{8} \mathrm{~B}$ reaction have been measured using the neutron time-of-flight method. The results of our experiment are in agreement with earlier measurement using the same technique [38] showing the same discrepancy with the data coming from positron counting and reported in the original paper by Rubbia et al. [13]. Model calculations based on the ZR-KO-DWBA for the ground state are able to reproduce the order of magnitude of the measured reaction cross section. In order to understand the differences of the results using the two experimental methods there is a strong need to perform other experiments at the ${ }^{3} \mathrm{He}$ beam energy above $10 \mathrm{MeV}$.

\section{D. ${ }^{8} \mathrm{Li}$ cross section measurements}

The two-body reaction ${ }^{7} \mathrm{Li}+\mathrm{d} \rightarrow{ }^{8} \mathrm{Li}+\mathrm{p}$ is the only possible channel that leads to the production of protons and ${ }^{8} \mathrm{Li}$. Therefore, the angular distribution of ${ }^{8} \mathrm{Li}$ can be deduced from the angular distribution of the protons by using the conservation laws. The question that may remain open is whether the process is a transfer reaction (stripping reaction) or goes through an excited compound nucleus that eventually decays by proton evaporation (compound nucleus formation and decay). This question can possibly be disentangled by studying the symmetries of the proton angular distribution and the shape of the proton energy spectra which are supposed to be very different in those two opposite cases. However, the energetics is not affected by

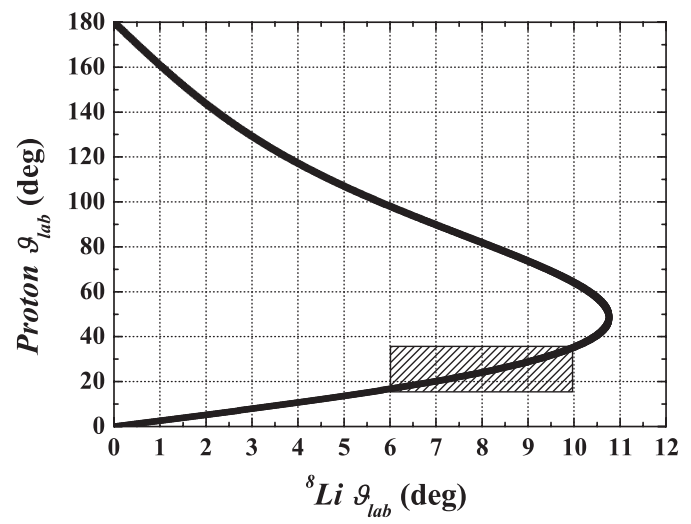

FIG. 20. Correlation of the protons laboratory angle with respect to the laboratory angle of ${ }^{8} \mathrm{Li}$. The hatched area highlights the region of maximum production of ${ }^{8} \mathrm{Li}$.

the details of the reaction process because it follows from the mass-energy conservation law.

Because of the reverse kinematics, ${ }^{8} \mathrm{Li}$ nuclei are strongly focused in the forward direction, while protons are spread out over $4 \pi$. The angular correlation expected by using linear momentum and energy conservation laws is shown in Fig. 20. The solid curve shows that the maximum laboratory angle expected for ${ }^{8} \mathrm{Li}$ with respect to the beam direction is $\approx 11^{\circ}$, which corresponds to protons emitted at about $50^{\circ}$. Figure 21 shows the expected correlation $E_{\text {lab }}$ vs $\vartheta_{\text {lab }}$ (lab energy vs angle with respect to the beam direction in the laboratory reference frame) for the case of ${ }^{8} \mathrm{Li}$ produced in its ground state. The curve shows that the laboratory energy of ${ }^{8} \mathrm{Li}$ is between 11 and $24 \mathrm{MeV}$. Protons can be produced instead with a maximum energy of about $13 \mathrm{MeV}$. From the above considerations, if follows that it is sufficient to measure the angular distribution of the protons to obtain the angular distribution of ${ }^{8} \mathrm{Li}$, regardless of the kind of reaction process.

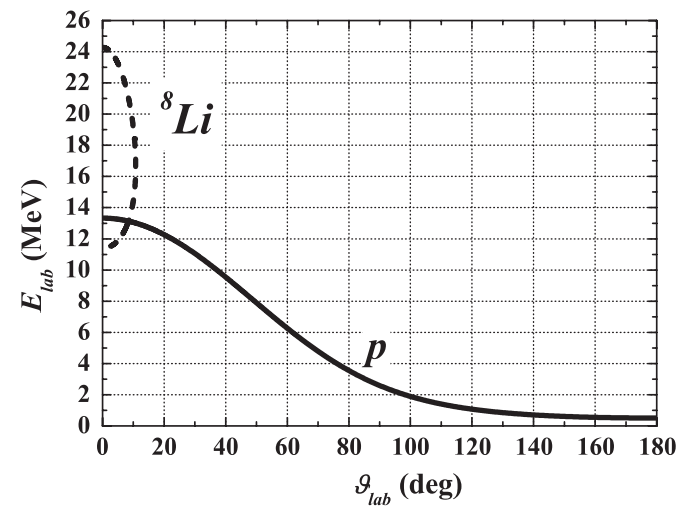

FIG. 21. The expected laboratory energy vs laboratory angle correlations for protons (solid line) and ${ }^{8} \mathrm{Li}$ (dashed line) from the two-body kinematics. The calculation is performed considering ${ }^{8} \mathrm{Li}$ in the ground state. 


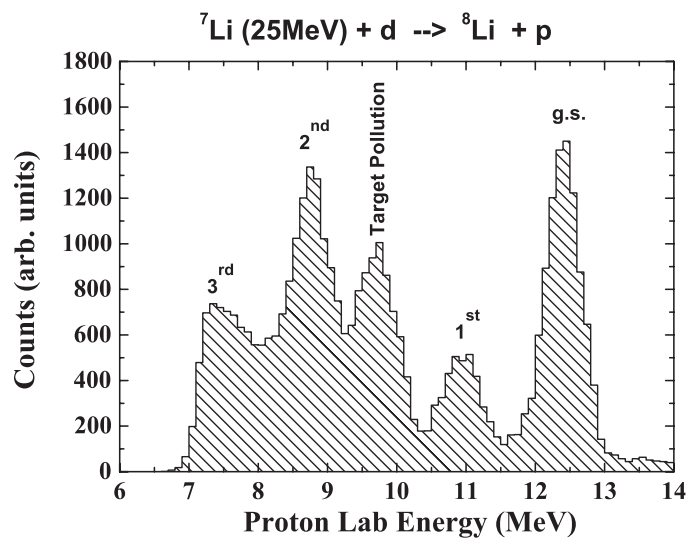

FIG. 22. Proton laboratory energy spectrum measured at $20.6^{\circ}$ with respect to the beam.

\section{Experimental method and results}

In the experiment performed at LNL, a pulsed beam of ${ }^{7} \mathrm{Li}$ of $25 \mathrm{MeV}$ was produced by the XTU Tandem at LNL. The target was of the $C D_{2}$ type. Protons were detected by the $8 \pi L P$ apparatus [47] which is a $4 \pi$ detector made out of more than 300 two-stage $\Delta E-E$ telescopes. The main duty of $8 \pi L P$ is to detect and identify light charged particles, namely, protons, deuterons, tritons and $\alpha$ particles.

In Fig. 22 the proton energy spectrum measured at the laboratory angle of $20.6^{\circ}$ is shown. Five peaks can be readily seen. The lower energy peak is partially cut because of the energy threshold of the detector.

In order to interpret the origin of these five peaks it is necessary to consider the energy balance of the reaction, namely, the connection between the $Q$ values and the known level scheme of ${ }^{8} \mathrm{Li}$. The peaks correspond to the reaction in which ${ }^{8} \mathrm{Li}$ is in its ground state (consequently maximum allowed kinetic energy for the protons), 1st, 2nd and 3rd excited state. The highest is the energy of the excited state, the lowest is the kinetic energy of the protons. An additional peak is observed due to the elastic scattering of ${ }^{7} \mathrm{Li}$ on the hydrogen as a contaminant of the target.

There are no experimental points for the 3rd exited state case because of the energy cut due to the detector's energy thresholds (see Fig. 23). The good agreement between the points and the curves in Fig. 23 supports the correct assignment of the origin of the peaks.

The laboratory angular distribution of the protons correlated to ${ }^{8} \mathrm{Li}$ produced in the ground state is shown in Fig. 24. Protons are mostly produced in an angular range lower than $40^{\circ}$. By going back to Fig. 20, this means that ${ }^{8} \mathrm{Li}$ production is maximized at the laboratory angles between $6^{\circ}$ and $10^{\circ}$. This region is highlighted with a hatched area.

In order to extract the absolute cross section, the lab angular distribution in Fig. 24 has been normalized to cross section units by using the elastic scattering and transformed

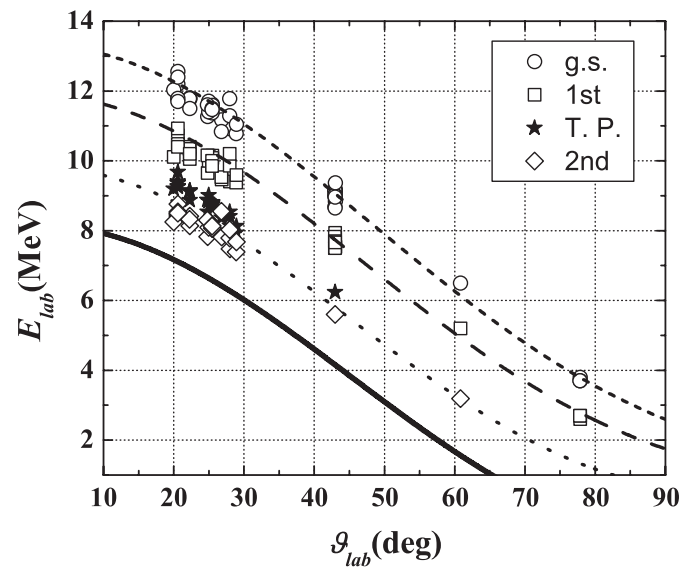

FIG. 23. Comparison between the measured energy peak at different laboratory angles and the values (solid lines) expected from two-body kinematics and different excited states of ${ }^{8} \mathrm{Li}$.

into the center of mass (c.m.) frame. The c.m. distribution is shown in Fig. 25 along with the results of Ref. [48]. The label " $8 \mathrm{MeV}$ " is here used since a beam of $25 \mathrm{MeV}$ of ${ }^{7} \mathrm{Li}$ on deuterons gives rise to the same reaction c.m. angular distribution of a deuteron beam of $\approx 8 \mathrm{MeV}$ impinging on a ${ }^{7} \mathrm{Li}$ target (direct kinematics). The data from Ref. [48] refer to a deuteron beam of $12 \mathrm{MeV}$ impinging on a ${ }^{7} \mathrm{Li}$ target. The angle integrated cross section is obtained by the following numerical integration:

$$
\sigma=\int \frac{d \sigma}{d \Omega} d \Omega
$$

For the present case, the integration was limited to the angular range of the data. This means that the total ${ }^{8} \mathrm{Li}$ cross section obtained of $89 \pm 18 \mathrm{mb}$ is a lower limit. This datum is plotted in Fig. 26 (full square) along with the cross section from Ref. [49] (empty circles) and Ref. [48] (empty triangle). Considering that only the ground state of ${ }^{8} \mathrm{Li}$ has been included in the experimental cross section, the present

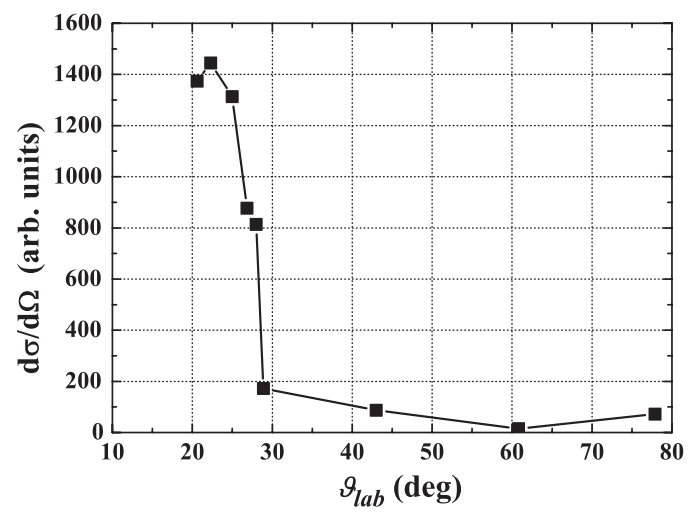

FIG. 24. Angular distribution in the laboratory reference frame of the protons corresponding to ${ }^{8} \mathrm{Li}$ in the ground state. 


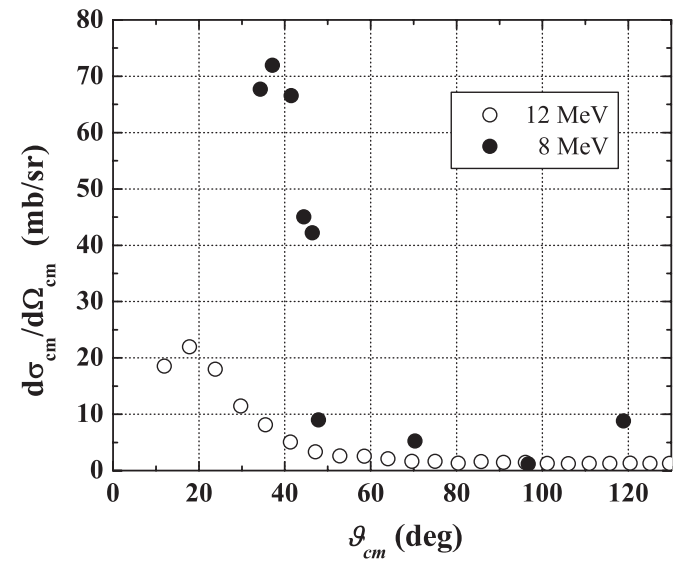

FIG. 25. Center of mass angular distribution for the protons corresponding to ${ }^{8} \mathrm{Li}$ in the ground state.

datum is in rather good agreement with the other data taken from the literature.

\section{Summary}

The primary goal of this experiment was the measurement of the angular distribution of the ${ }^{8} \mathrm{Li}$ produced in the reaction $d\left({ }^{7} \mathrm{Li}, p\right)^{8} \mathrm{Li}$ at $25 \mathrm{MeV}$. The experimental method takes advantage of the two-body nature of the process. ${ }^{8} \mathrm{Li}$ angular distribution is obtained by measuring the angular distribution of the protons. A by-product of this experiment is the total ${ }^{8} \mathrm{Li}$ production cross section. Considering that only the ground state is included, the cross section at $25 \mathrm{MeV}$ is in good agreement with data from literature.

\section{E. Other alternatives for production}

Another option for ${ }^{8} \mathrm{Li}$ and ${ }^{6} \mathrm{He}$ radioactive ion beam (RIB) is the two-stage production scheme [50] that can best be applied at the new generation of high current deuteron beam linacs, such as Soreq Applied Research Accelerator

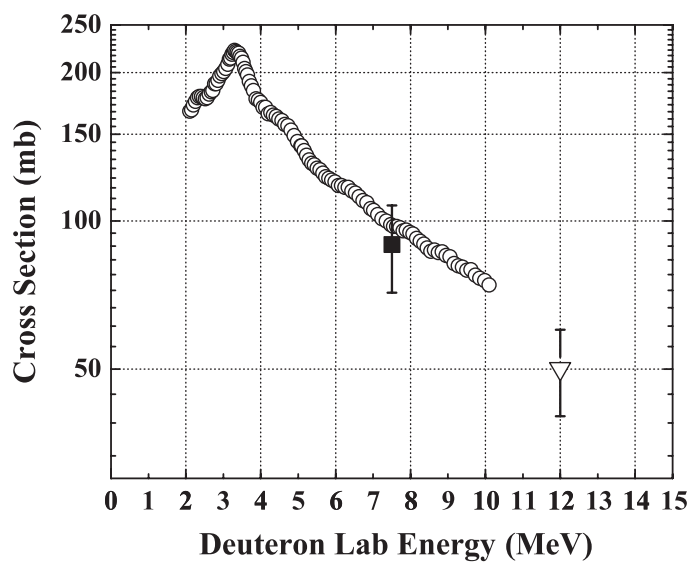

FIG. 26. ${ }^{8} \mathrm{Li}$ production cross section compared with known data. Full square: present experiment; empty circles from Ref. [49]; empty triangles from Ref. [48]
Facility (SARAF), [51], IFMIF [52] and SPIRAL2 [53]). See also the ${ }^{6} \mathrm{He}$ chapter above and [50]. A primary target (the neutron converter) is bombarded with an intense deuteron beam, providing a fast and directed neutron spectrum, whereas the actual isotope production takes place in a secondary target by fast neutron-induced reactions.

Extensive Monte Carlo simulations using a deuteron beam of $40 \mathrm{MeV}$ predict a high production yield of $2.5 \times$ $10^{12}{ }^{6} \mathrm{He} / \mathrm{mC}$ and $0.9 \times 10^{12}{ }^{8} \mathrm{Li} / \mathrm{mC}$. Even after considering extraction and ionization issues, these yields are at least an order of magnitude higher than is available today, and may be compatible with $\beta$ beam required yields, by considering the appropriate deuterons beam current. In recent years, an extensive experimental research has been carried out to validate the simulation predictions and to construct for the first time a full two-stage system.

A first fully optimized apparatus was constructed at SARAF phase I, for the production of intense ${ }^{8} \mathrm{Li}$ RIB following the lines above [54]. The ${ }^{8} \mathrm{Li}$ isotopes are produced in an encapsulated porous $\mathrm{B}_{4} \mathrm{C}$ target that is heated in a custom-built high temperature vacuum furnace. This apparatus will facilitate, for the first time, a direct measurement of the total efficiency of the proposed irradiation scheme.

High effectuality of the two-stage irradiation process, along with the mature technology of high-current deuteron linacs and the corresponding fast-neutron converters, may allow the fulfillment of a production scheme for the $\beta$ beam in the next few years at a rather low cost and without high-risk development issues.

\section{F. Summary of isotope production for beta beam isotopes}

Table III below shows the rates that can be achieved today. ${ }^{6} \mathrm{He}$ and ${ }^{18} \mathrm{Ne}$ have been experimentally verified. Rates for ${ }^{8} \mathrm{Li}$ and ${ }^{8} \mathrm{~B}$ have been simulated, using available information on cross sections and by optimizing the production ring target wedge and the incoming ion beam. We see that the production ring still needs some tuning to perform as specified. Considerations concerning the

TABLE III. The rate (r) extracted from the source using different production methods $\left({ }^{6} \mathrm{He}\right.$ estimated from experiments, for ${ }^{18} \mathrm{Ne}$ from experiments and calculations and rates for ${ }^{8} \mathrm{Li}$ and ${ }^{8} \mathrm{~B}$ are estimated from calculations).

\begin{tabular}{lcccc}
\hline \hline Isotope & ${ }^{6} \mathrm{He}$ & ${ }^{18} \mathrm{Ne}$ & ${ }^{8} \mathrm{Li}$ & ${ }^{8} \mathrm{~B}$ \\
\hline Production & ISOL(n) & ISOL & P-ring & P-ring \\
Beam & SPL(p) & Linac4(p) & d & ${ }^{3} \mathrm{He}$ \\
I $[\mathrm{mA}]$ & 0.07 & 7 & 0.160 & 0.160 \\
$\mathrm{E}[\mathrm{MeV}]$ & 2000 & 160 & 25 & 25 \\
$\mathrm{P}[\mathrm{kW}]$ & 140 & 1120 & 4 & 4 \\
Target & $\mathrm{W} / \mathrm{BeO}$ & ${ }^{23} \mathrm{Na},{ }^{19} \mathrm{~F}$ & ${ }^{7} \mathrm{Li}$ & ${ }^{6} \mathrm{Li}$ \\
$\mathrm{r}\left[10^{13} / \mathrm{s}\right]$ & 5 & 1.0 & 0.1 & 0.08 \\
\hline \hline
\end{tabular}


collection device and related problems (see III B) have not been considered for ${ }^{8} \mathrm{Li}$ and ${ }^{8} \mathrm{~B}$ in the simulations.

\section{IONIZATION: THE ECR SOURCE}

The high frequency electron cyclotron resonance (ECR) ion source is expected to accept an intense continuous flux of ${ }^{6} \mathrm{He}$ or ${ }^{18} \mathrm{Ne}$, ionize the gas and bunch the ions with a high efficiency. As a continuation of the work started in the EURISOL Design Study, a compact, robust, innovative design was proposed for a $60 \mathrm{GHz} \mathrm{ECR}$ ion source prototype called SEISM: Sixty gigahertz ECR ion source using megawatt magnets. Using high field magnets technology, the confinement structure was constructed and tested. Upcoming experiments at $28 \mathrm{GHz}$ will allow an estimation of SEISM beam characteristics, compared to beams extracted from the known $28 \mathrm{GHz}$ ECR ion sources.

\section{A. Ion source specifications}

As described previously (see Sec. III F) radioactive ion beam intensities of up to $5 \times 10^{13}$ ions per second for ${ }^{6} \mathrm{He}$ (i.e., $8 \mathrm{p} \mu \mathrm{A}$ ) are foreseen. The beam should be structured according to the postacceleration duty cycle (refer to V): as a working hypothesis one considered short pulses of 50 to $100 \mu$ s duration with a 10 to $25 \mathrm{~Hz}$ repetition rate.

Due to the high ionic densities in a classical electron cyclotron ion source (ECRIS) (up to $10^{13}$ charges per $\mathrm{cm}^{3}$ for a $28 \mathrm{GHz}$ plasma $[55,56])$ and to its high ionization efficiency for noble gases (close to 100\%), ECRIS allows the production of intense continuous beams and is considered as a promising solution.

Studies started within the EURISOL Design Study $[57,58]$ predicted that short bunches of $100 \mu$ s duration could be produced in the pulsed working mode called preglow (PG) [59], provided that the heating radiofrequency would be much higher than $28 \mathrm{GHz}$.

Experiments have been performed at LPSC Grenoble with a Phoenix V2 ECRIS in PG mode at $18 \mathrm{GHz}$ and at $28 \mathrm{GHz}$, and a theoretical model has been developed in collaboration with IAP Nizhny Novgorod [60-62], confirming that increasing the heating frequency would allow the production of higher intensities in PG mode.

The location of the ion source, close to the target, will impact its lifetime due to the high radioactivity level, therefore the magnetic structure should be radiation hard.

A $60 \mathrm{GHz}$ ECRIS prototype, the first in the world, was designed at LPSC Grenoble with the aim of fulfilling the specifications listed above.

\section{B. Design choices}

As a first design, the simplest magnetic configuration, a cusp structure, was chosen. Extensive simulations have shown that two sets of coils supplied with opposite currents of $30 \mathrm{kA}$ could generate a closed $2.1 \mathrm{~T}$ iso-B surface for $60 \mathrm{GHz}$ resonance (Fig. 27), with magnetic field values up to

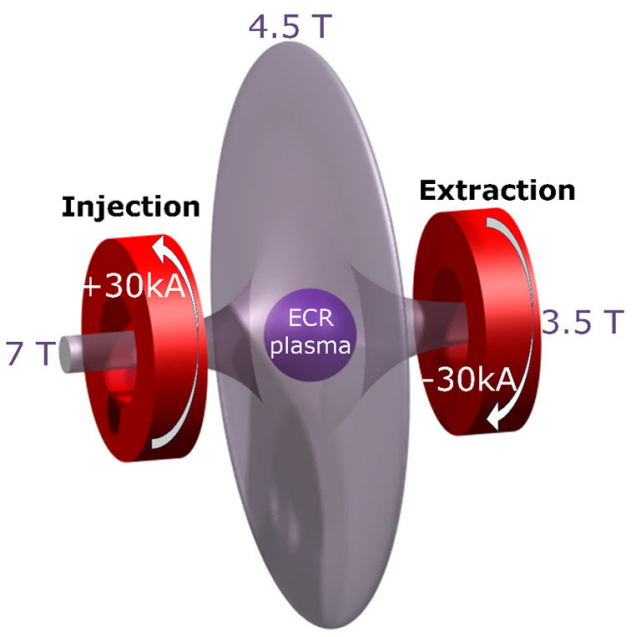

FIG. 27. Schematic view of the SEISM cusp magnetic trap.

$7 \mathrm{~T}$ at the injection and $3.5 \mathrm{~T}$ at the extraction on a $100 \mathrm{~mm}$ axial mirror length, and $4.5 \mathrm{~T}$ for the radial mirror [63].

In order to reach high magnetic field gradients on such short dimensions, radiation-hard coils using Laboratoire National des Champs Magnétiques Intenses (LNCMI) polyhelix technology (see [64]) were used, accepting current densities up to $640 \mathrm{~A} / \mathrm{mm}^{2}$. Due to their low resistivity, the coils need $6 \mathrm{MW}$ electrical power and can be cooled with deionized water. Following the magnetic field calculations, thermal and hydraulic calculations were performed using a general finite element solver program in order to optimize polyhelix cooling. The helices are radially cooled, so the windings are stuck together with 20 to 24 pieces of preimpregnated fiberglass (prepreg, see Fig. 28), in between which the water flows. At full power operation, temperature can locally reach $330^{\circ} \mathrm{C}$ and exceed the prepreg thermal resistance, so new insulator designs were investigated to prepare full power tests [65]. The cooling tanks (Fig. 29) were designed to bear the stress due to an internal water pressure of 43 bars and limit the lengthening due to the $300 \mathrm{kN}$ magnetic repelling forces.

The plasma chamber diameter is $60 \mathrm{~mm}$ due to the $80 \mathrm{~mm}$ helix inner diameter, with a shoulder at the center

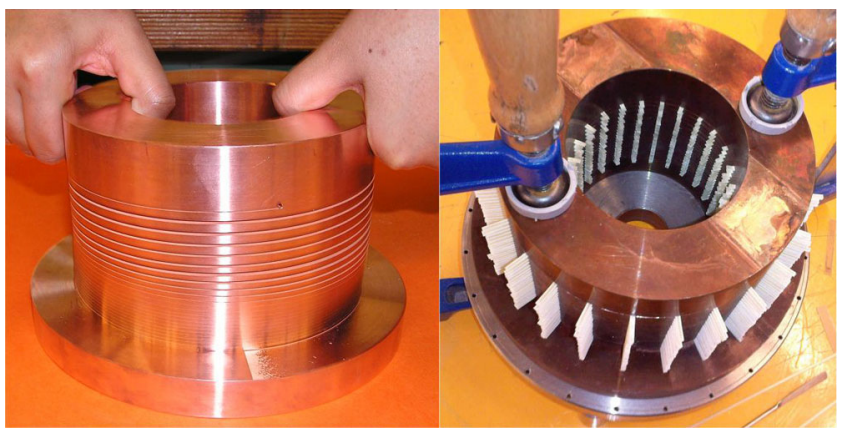

FIG. 28. Insertion of the prepreg insulators between the helix windings. 


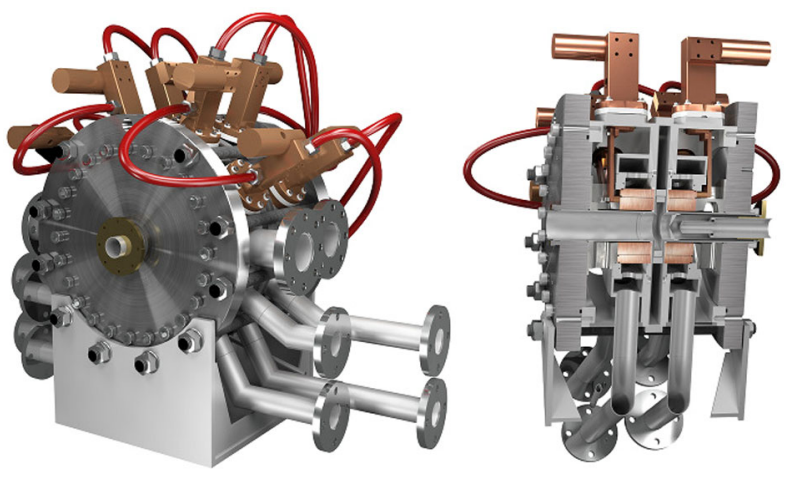

FIG. 29. Three-dimensional view of SEISM prototype.

that allows the magnetic field lines to pass from axial to radial mirrors through the resonance zone without touching the chamber walls. A polarized ring was added to prevent radial particle leaks. The plasma chamber is insulated by $2 \mathrm{~mm}$ thick PEEK parts. As a first approach, conventional single-gap plasma and puller electrodes were designed. Depending on the first experimental results, a multielectrode design will be performed in order to extract high intensities at high voltage (above $50 \mathrm{kV}$ ).

\section{Experimental validation}

Tests were conducted at LNCMI to measure the magnetic field map of the SEISM confinement structure [66-69]. Axial and radial hall probes allowed $1 \mathrm{~mm}$-step measurements, all along three axes parallel to the chamber central axis, at 0,15 , and $30 \mathrm{~mm}$ radial distances. Precise positions of the helices magnetic centers relatively to the plasma chamber center were verified by a flux variation integration experimental method. One could see that the $1 \mathrm{~T}$ iso-B surface corresponding to the $28 \mathrm{GHz}$ resonance zone is closed at $15 \mathrm{kA}$ (see Fig. 30).

However, one observed that the peak to peak length was about $90 \mathrm{~mm}$, so shorter than calculated. As a consequence,

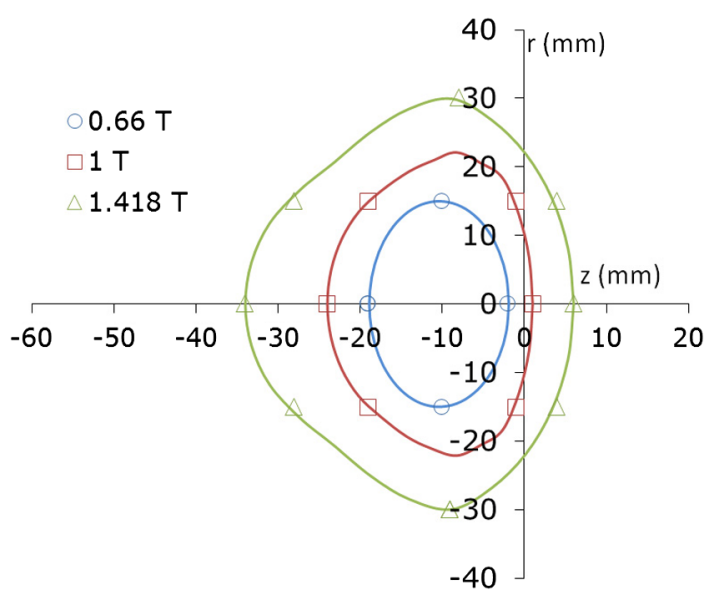

FIG. 30. Magnetic field map for $15 \mathrm{kA}$ current intensity on the coils.

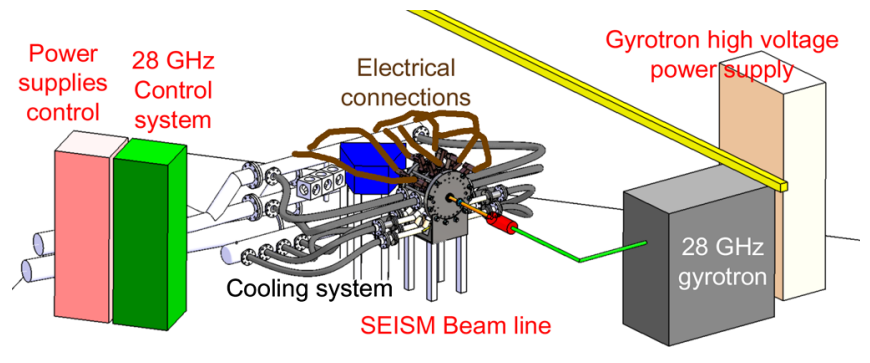

FIG. 31. Schematic view of the beam experiment layout.

the maxima value is up to $20 \%$ lower than expected on the extraction side. Moreover the cusp point, where the magnetic field value is zero, is located $9 \mathrm{~mm}$ further towards the extraction. Such displacement could cause energetic electrons to follow the magnetic field lines and hit the chamber, rapidly creating a hole.

In order to solve this issue and to prepare plasma experiments, possible intensity operating ranges are under evaluation. The adjustment of the ratio of applied currents between injection and extraction coils is computed in order to bring back the zero field point at the centre of the plasma chamber. In the meantime, parametric simulations, including for example, the temperature gradient in the cooling water or mesh variations [70], were introduced to fit the discrepancies between the calculations and the measurements.

\section{Perspectives}

The next step is to produce a $28 \mathrm{GHz}$ plasma in order to compare SEISM cusp performances to existing minimumB ECRIS [71,72]. Figure 31 shows the beam line layout which is currently under construction at LNCMI.

Depending on the beam characteristics, the pertinence of producing a $60 \mathrm{GHz}$ plasma with this first prototype will be evaluated. Developments are already ongoing to overcome the thermal limitation on the prepreg insulators. The design could also be complexified towards a minimum-B magnetic structure, as first preliminary design studies show the possibility to use polyhelix technology to produce a multipolar radial field.

A $60 \mathrm{GHz}-300 \mathrm{~kW}$ gyrotron was developed at IAP within an ISTC contract [73] and should be available at LPSC-LNCMI, end of summer 2012. In the future, in the frame of the COLOSSECRIS excellency laboratory project, LNCMI power supplies may provide high intensity currents to magnet experiments at research facilities such as ILL, ESRF and LPSC. Possible $60 \mathrm{GHz}$ experiments could then benefit from a high intensity beam line currently being developed at LPSC.

\section{THE ION LINAC}

Due to the low duty cycle of $0.05 \%$ and the high pulse beam current up to $13 \mathrm{~mA}$ as required for the beta beam, the 


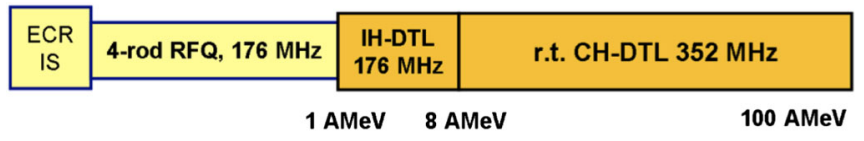

FIG. 32. Schematic layout of an IH/CH-DTL for the production of b-beams.

use of normal conducting rf structures even up to the intended final energy of $100 \mathrm{MeV} / \mathrm{u}$ has been considered a favorable choice. Even at accelerating gradients between 3 and $6 \mathrm{MV} / \mathrm{m}$ the thermal load on the cavities is very moderate. For the desired maximum mass to charge ratio of $A / q=3$ an operating frequency of $175 \mathrm{MHz}$ at energies up to $10 \mathrm{MeV} / \mathrm{u}$ is well adapted, which then would be changed to $352 \mathrm{MHz}$ for further acceleration [74].

Figure 32 shows a schematic layout of a possible ion linac and the parameters are shown in Table IV. The first accelerating stage behind the ECR ion source would be a radio-frequency quadrupole (RFQ). However, the ions are not fully stripped as assumed in [74] but have charge state +1 , therefore we may consider two RFQs and strip the ions fully before the drift tube linac (DTL) structure. The study of these RFQs still remains to be done. The transition energy between RFQ and the DTL has been chosen to be $1 \mathrm{MeV} / \mathrm{u}$, which can be adapted to DTL requirements during further optimization procedures.

Possible candidates for NC-DTLs are H-mode cavities (Crossbar H-type cavities, CH-DTLs, and Interdigital Hmode cavities, IH-DTLs). In general, H-mode cavities have high shunt impedance. IH structures are operated in the low energy regime between 0.1 and $10 \mathrm{MeV} / \mathrm{u}$ with rf frequencies between 36 and $250 \mathrm{MHz}$. The IH-DTL structure has no competitor with respect to rf efficiency. The first DTL part could consist of three IH structures operated at $176 \mathrm{MHz}$. The input energy is $1 \mathrm{MeV} / \mathrm{u}$ and the output energy $8 \mathrm{MeV} / \mathrm{u}$. The total voltage gain is $22 \mathrm{MV}$ with a length of about $7 \mathrm{~m}$. The total required power per cavity including the beam loading is between 600 and $750 \mathrm{~kW}$. The following DTL section from 8 to $100 \mathrm{MeV} / \mathrm{u}$ could consist of NC-CH cavities. $\mathrm{CH}$ cavities are operated in the $\mathrm{H} 21$ mode. The $\mathrm{CH}$ section would be operated at $352 \mathrm{MHz}$. For this frequency cost-efficient klystrons in the MW range are available. To cover the voltage gain of $276 \mathrm{MV}$ about 46 $\mathrm{CH}$ structures are necessary. The effective voltage per cavity varies between 5 and $7 \mathrm{MV}$. The total power per

TABLE IV. Beta-beam requirements for the ion linac.

\begin{tabular}{lc}
\hline \hline Beta beam linac parameters & \\
\hline Duty cycle & $0.05 \%$ \\
Beam current & $13 \mathrm{~mA}$ \\
Mass to charge ratio A/q & 3 \\
Input energy $W_{\text {in }}$ & $8 \mathrm{keV} / \mathrm{u}$ \\
Output energy $W_{\text {out }}$ & $100 \mathrm{MeV} / \mathrm{u}$ \\
Input emittance $\epsilon_{\text {in,rms,normalized }}$ & $0.2 \pi \mathrm{mm} \mathrm{mrad}$ \\
\hline \hline
\end{tabular}

cavity is kept below $1 \mathrm{MW}$ including the beam loading. The length of the CH-DTL is about $100 \mathrm{~m}$. The total length is about $110 \mathrm{~m}$. This corresponds to a real estate gradient of $2.73 \mathrm{MV} / \mathrm{m}$.

\section{THE RAPID CYCLING SYNCHROTRON}

The work on the RCS [6] was done within the FP6 Framework Program (EURISOL Design Study) [12] and it is summarized here for completeness.

\section{A. RCS general parameters}

The RCS accelerates $\mathrm{He}$ and $\mathrm{Ne}$ ion beams from $100 \mathrm{MeV} / \mathrm{u}$ to a maximum magnetic rigidity of 14.47 $\mathrm{Tm}$ (that is the rigidity of $3.5 \mathrm{GeV}$ protons, $787 \mathrm{MeV} / \mathrm{u}$ for ${ }^{6} \mathrm{He}^{2+}$ and $1.65 \mathrm{GeV} / \mathrm{u}$ for ${ }^{18} \mathrm{Ne}^{10+}$ ) with a repetition rate of $10 \mathrm{~Hz}$. The threefold symmetry lattice proposed is based on FODO cells, i.e., lattice cells having interleaved focusing and defocusing magnets and drift spaces in between, with missing magnets providing three achromatic arcs and three sufficiently long straight sections for accommodating the injection system, the high energy fast extraction system and the accelerating cavities. The number of dipoles have been optimized to obtain a transition energy allowing acceleration of protons up to $3.5 \mathrm{GeV}$. The dipoles have been split into two parts separated by a drift space to place absorbers to intercept the decay products. The physical radius has been adjusted to $40 \mathrm{~m}$ in order to facilitate the synchronization between the CERN PS and the RCS and therefore the transfer of bunches from one ring to the other. The ring is composed of 60 short dipoles and 48 quadrupoles. A schematic view of the RCS layout is shown in Fig. 33, left, and the main parameters are summarized in table V.

\section{B. Optical design}

The RCS is partitioned into 24 FODO cells, six in arcs and two in a straight section. The betatron phase advance per cell (i.e., quadrupole strength) and the length of the two sections without dipoles in the arcs have been adjusted so as to cancel the dispersion function in long straight sections and to obtain, with only two quadrupoles families, a

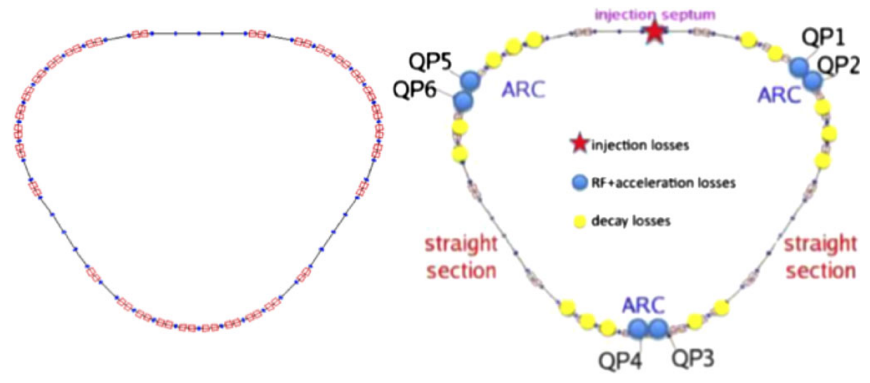

FIG. 33. Schematic layout the RCS, left, and the points where the highest losses are, right. 
TABLE V. Beta-beam requirements for the RCS.

\begin{tabular}{lc}
\hline \hline Beta-beam RCS parameters & \\
\hline Circumference & $251 \mathrm{~m}$ \\
Superperiodicity & 3 \\
Injection energy & $100 \mathrm{MeV} / \mathrm{u}$ \\
Maximum magnetic rigidity & $14.47 \mathrm{Tm}$ \\
Repetition rate & $10 \mathrm{~Hz}$ \\
Number of dipoles & 60 \\
Number of quadrupoles & 48 \\
Max ramping rate & $24 \mathrm{~T} / \mathrm{s}$ \\
Emittance h/v & $72 / 39 \pi \mathrm{mm} \mathrm{mrad}$ \\
\hline \hline
\end{tabular}

working point located in a region of the tune diagram which is free of systematic resonances up to the fourth order. Dipoles are only $1.4 \mathrm{~m}$ long with a maximum magnetic field of $1.08 \mathrm{~T}$ to avoid a high ramping rate for the $10 \mathrm{~Hz}$ operation. The quadrupoles are $0.4 \mathrm{~m}$ long and have a maximum gradient of less than $11 \mathrm{~T} / \mathrm{m}$. The diluted transverse emittances in the RCS after multiturn injection are calculated from the emittances required in the PS at the transfer energy with a possible blowup of $20 \%$.

\section{Injection}

The ion source delivers a beam pulse of $50 \mu \mathrm{s}$. The revolution period at $100 \mathrm{MeV} / \mathrm{u}$ is $1.96 \mu \mathrm{s}$, and the injection process takes place over 26 turns (multiturn injection) in one of the long straight sections by means of an electrostatic septum and two pulsed kickers. Optimum filling in the horizontal phase space is achieved when the incoming ions are injected with a position and a slope which minimize their Courant and Snyder invariant. In the vertical phase space the dilution is obtained by a betatron function mismatch and a beam position offset. The injection efficiency is $80 \%$.

\section{Acceleration}

After injection the circulating beam is continuous and occupies a rectangle in the longitudinal phase space. To capture the injected beam, one stationary bucket is created. During trapping, the magnetic field is clamped at its minimum value for a period of a few $\mathrm{ms}$ and the synchronous phase is zero. The rf voltage is optimized to obtain a beam rotation of about $90^{\circ}$ and a momentum spread as small as possible before the acceleration of the 20 bunches starts. The 20 bunches are then single turn extracted and transferred to the PS. The accelerating voltage is $100 \mathrm{kV}$ over a frequency range of 0.51 to 1.12 MHz. Ferrite loaded cavities developed at CERN or magnetic alloy cavities like those developed for SIS18 at GSI or at J-Parc could would be a possible choice.

\section{E. Other beam optics aspects}

Several beam dynamics studies have been investigated in order to assess the feasibility of the RCS, in addition to the results presented here. Unavoidable magnet misalignments and dipole field errors can affect the RCS closed orbit. Distortions to be expected have been statistically estimated assuming standard error tolerances and a correction system has been defined. In fast ramping machines such as the beta-beam RCS, eddy currents induced in metallic vacuum chamber walls by the time varying magnetic field produce various field components acting on the beam. In dipole vacuum chambers, one important component is a sextupole which modifies the natural chromaticity of the ring. The associated effects may be compensated for to guarantee the beam performance. The beam dynamics studies made on the RCS show that the ring can be realized using available technologies.

\section{F. Vacuum system requirements for the RCS}

The vacuum decrease due to beam losses is a potential problem for all machines and it has been studied for the RCS. The required gas pressure for a good transmission is $1 \times 10^{-8}$ mbar.

The losses are dominated by radioactive decay. However, beam losses at injection are crucial for the transmission. Transmission calculations assume a beam loss at injection of $20 \%$. Using ${ }^{18} \mathrm{Ne}^{10+}$ as projectile ion the pressure stays below $1 \times 10^{-8}$ mbar for pumping speeds greater than $2 \mathrm{~m}^{3} / \mathrm{s}$, reaching a maximum for effective pumping speed $S_{\text {eff }}=5 \mathrm{~m}^{3} / \mathrm{s}$ of about $6.5 \times 10^{-9}$ mbar. The situation for ${ }^{6} \mathrm{He}^{2+}$ is different. For pumping speeds less than $10 \mathrm{~m}^{3} / \mathrm{s}$ the pressure goes up exceeding the $1 \times 10^{-8}$ mbar limit. The pumping speed is not sufficient to remove enough of the gas desorbed at injection before the next cycle starts. As it is desirable to have a conventional pumping system installed in the RCS, the best way to work around this problem would be to reduce the beam losses at injection. If it is possible to reduce the losses to about $10 \%$ an effective pumping speed of $2 \mathrm{~m}^{3} / \mathrm{s}$ would be sufficient to stay below the $1 \times 10^{-8}$ mbar limit. If the injection losses cannot be reduced, one has to consider an increase of the pumping speeds for example by applying a NEG coating [75] to a part of the vacuum chamber. This analysis was done for ${ }^{6} \mathrm{He}^{2+}$ only, since the pressure evolution for ${ }^{18} \mathrm{Ne}^{10+}$ is not critical. Because most beam losses occur within or close to the dipole magnets, NEG coating should be applied to these dipoles. For all calculations the maximum residual gas pressure is $5 \times 10^{-9}$ mbar, while the effective pumping speed due to the NEG ranges from about 310 to $650 \mathrm{~m}^{3} / \mathrm{s}$ for 8 and 20 coated dipoles respectively. One has to consider an ongoing saturation effect, which reduces the pumping speed of the NEG coating over time. If NEG coating is needed all dipole magnets should be treated with NEG to ensure a stable residual pressure over the entire time of operation. It is important to remark that only beam losses inside the RCS ring are relevant for the calculation of the pressure bump at injection. In this sense a beam loss of $20 \%$ means that all particles are lost inside the machine. Losses outside the machine, e.g., inside a drift line just 
TABLE VI. Beam loss for ${ }^{6} \mathrm{He}\left({ }^{18} \mathrm{Ne}\right)$.

\begin{tabular}{lccc}
\hline \hline $\mathrm{E}[\mathrm{MeV} / \mathrm{u}]$ & Injection & Decay & rf \\
\hline 100 & $30 \%$ & $0.10(0.45) \%$ & $5.70(9.40) \%$ \\
$400(640)$ & $\cdots$ & $0.80(0.20) \%$ & $2.85(8.50) \%$ \\
$787(1650)$ & $\cdots$ & $1.80(0.70) \%$ & $4.75(5.05) \%$ \\
\hline \hline
\end{tabular}

before injection do not contribute to the pressure rise and must be subtracted. In general it is hard to distinguish these two effects.

\section{G. Radiation protection studies}

Detailed radiation protection studies were realized according to the different loss mechanisms within the RCS. They permitted to define the shielding required by the machine operation, the classification of the area and limits on the release of airborne activity. Beam losses can be divided into injection, decay and rf (capture and acceleration) losses, see Table VI. At injection 30\% of the beam is lost on the septum. Decay losses are uniformly distributed in the dipoles and in the short straight sections in the arcs during all the magnetic cycle. Radio-frequency losses are point losses that occur in the families of quadrupoles in the arcs as indicated in Fig. 33, to the right.

The areas around the RCS tunnel will probably be classified as supervised radiation areas during operation, with a dose rate constraint of $3 \mu \mathrm{Sv} / \mathrm{h}$ : this would require concrete shielding thicknesses ranging from 3 to $5 \mathrm{~m}$, depending on the position in the tunnel. In these places where different kinds of losses occur, the thickness imposed by the dominating mechanism was considered. In the released airborne activity study a constant rate of $10000 \mathrm{~m}^{3} / \mathrm{h}$ was chosen for the ventilation system in the RCS tunnel. In this condition, the effective dose given to the reference population in one year of operation was estimated to be in the order of $0.7 \mu \mathrm{Sv}$ for the most critical ion, i.e., ${ }^{18} \mathrm{Ne}$. It is well below the reference value for CERN emission. Table VII presents the contributions of the main radionuclides to the annual effective dose.

For the inhalation dose to workers that could access the tunnel during shutdown periods a conservative assumption was made: the ventilation system is not operating. The intervention time depends on dose rates and on whether or not the ventilation system is on. For a one hour intervention, the integrated dose does not exceed the constraints, considerably below $1 \mu \mathrm{Sv}$ even without waiting time. Furthermore, if the ventilation system is working a waiting

TABLE VII. Annual effective dose to the reference population: contributions from the main radionuclides in $\mu \mathrm{Sv}$.

\begin{tabular}{lcccc}
\hline \hline N-13 & Ar-41 & C-11 & O-15 & Be-7 \\
\hline 0.31 & 0.13 & 0.12 & 0.08 & 0.003 \\
\hline \hline
\end{tabular}

time before access of nearly 20 minutes is enough to completely change the air in the tunnel.

Dose rates from material activation were calculated for a three-month continuous operation and three different waiting times of one hour, one day and one week. The results show that, according to CERN area classification, the RCS tunnel is likely to be classified as a limited stay area, accessible one week after the shutdown. The doses do not decrease significantly after one week because the residual radionuclides that mostly contribute to the total dose have half-lives longer than one week. The high activation of the machine elements that remains after one week may require a remote handling system for the maintenance.

\section{THE CERN PS}

Ion acceleration in the PS and SPS is a routine operation since many years. Different ion types from light ions such as sulfur up to heavy ions such as lead have been accelerated. Studies of a possible acceleration scenario and the PS vacuum were performed during the EURISOL Design Study (see [12], for details). The recent measurements of the oscillation angle $\theta_{13}$ show that the requirements for short and intense bunches in the final accelerator, the decay ring, can be relaxed now. The reason for the requirement for a small "duty factor" (the time during which the intensity is distributed with respect to the total time the machine is working) is the signal to noise (atmospheric neutrinos) relation in the detector. The new relaxed bunching conditions in the decay ring have only been studied partly and would need reconsidering of the $\mathrm{rf}$ also in the PS. The original scenario in the PS that would give a sufficient rate of neutrinos for physics will be summarized here.

Since the beta-decay lifetime at injection in the PS is much longer than the cycle time of the RCS, it has been chosen to operate the PS at the rf harmonic consistent with the $10 \mathrm{MHz}$ upper frequency limit of the accelerating cavities and to transfer the maximum number of batches from the RCS. Thus, the PS harmonic of choice becomes $h=21$ and 20 bunches are accumulated one by one from the RCS. One rf bucket is left empty to accommodate the extraction kicker rise time.

The beta decay diminishes the number of ions accumulated on the PS injection plateau. Also the intensity of the first bunch injected into the PS will be less than the last. As little as $40 \%$ of the first helium bunch remains when the last one arrives. The situation is better in the neon case due to its longer half-life and more advantageous charge-to-mass ratio. The PS extraction kicker gap is positioned differently within the bunch train, from batch to batch, in order to even out the bunches that are ultimately stored in the decay ring. The longitudinal emittance that the PS must deliver is $0.80 \mathrm{eV} \mathrm{s}$ in the case of helium ions and $1.8 \mathrm{eV} \mathrm{s}$ for neon. This implies matching voltages of $\cong 30 \mathrm{kV}$ and $\cong 10 \mathrm{kV}$, respectively, in order to provide the required bunch length 
of $20 \mathrm{~ns}$ at ejection. No bunch shortening gymnastics are required due to the proposed addition of a $40 \mathrm{MHz}$ rf system in the receiving SPS (see Sec. VIII).

\section{A. Vacuum system requirements for the PS}

Particle losses are dominated by radioactive decay. Losses due to charge exchange are negligible, as the cross sections for electron capture are very small. For both ${ }^{6} \mathrm{He}^{2+}$ and ${ }^{18} \mathrm{Ne}^{10+}$ the losses are spread over the whole ring. The transmission was checked by using an ideal cycle assuming the maximum number of particles coming from the RCS. Given these numbers there were $4.29 \times 10^{12}$ particles ejected. This is close to the desired number of $4.31 \times 10^{12}$.

There are 147 vacuum pumps along the PS ring, which were assumed to be equally distributed. Together with the given total pumping speed of $38 \mathrm{~m}^{3} / \mathrm{s}$, the simulation program STRAHLSIM calculates an effective pumping speed of about $11.5 \mathrm{~m}^{3} / \mathrm{s}$. The pressure stays well below $10 \times 10^{-8}$ mbar for both ${ }^{6} \mathrm{He}^{2+}$ and ${ }^{18} \mathrm{Ne}^{10+}$ when a pumping speed of $11.5 \mathrm{~m}^{3} / \mathrm{s}$ is assumed.

At an accelerator operation with ${ }^{6} \mathrm{He}^{2+}$ and ${ }^{18} \mathrm{Ne}^{10+}$ as projectile ions an effective pumping speed of $11.5 \mathrm{~m}^{3} / \mathrm{s}$ in the PS ring is sufficient. This pumping speed is delivered by the existing vacuum system of the PS. The minimum effective pumping speeds needed for a stable operation were estimated to be $9 \mathrm{~m}^{3} / \mathrm{s}$ and $7 \mathrm{~m}^{3} / \mathrm{s}$ for ${ }^{6} \mathrm{He}^{2+}$ and ${ }^{18} \mathrm{Ne}^{10+}$ respectively.

\section{B. CERN PS radiation protection studies}

Preliminary work within the EURISOL study has focused on two radiation protection aspects related to the operation of the PS as part of a beta-beam facility, namely the induced radioactivity in the magnets and the air activation. A complete study would also include an analysis of the existing shielding (in particular with respect to those points, where the shielding is relatively thin), the prediction of induced radioactivity in hot components like septum magnets and the activation of the cooling water of the magnets. At this stage it is not possible to perform such a detailed study because of the lack of information on the operation conditions and on the exact particle loss distribution. Nevertheless, during this study it was possible to assess the impact that the beta-beam operation would have on the radiation level expected during maintenance and on the release of radioactivity to the environment.

The PS bridge (the SS42 region of the CERN PS, near Goward Road) has been studied using the same geometry models as for the $2 \mathrm{GeV}$ proton beam, studied for a possible upgrade of the injector complex, for LHC [76]. The conclusion is that dose rates for beta beams are lower by a factor 3 for ${ }^{18} \mathrm{Ne}$ and by a factor 16 for ${ }^{6} \mathrm{He}$ compared to full proton intensities. The energy deposition on the septum blade (SMH42) is $1.0 \times 10^{-4}[\mathrm{GeV} / \mathrm{cm} 3 /$ primary $]$ for ${ }^{18} \mathrm{Ne}$. The energy deposition on the blade of the septum SM16 is higher by a factor of 3.7. No show stopper has been found for the beta beam, neither for radio protection nor for the equipment.

At the beginning of the annual shutdown period in 2008, radiation survey measurements of ambient dose equivalent rate were performed to gain information about the present radiation levels in the CERN PS. The survey measurements are done at $40 \mathrm{~cm}$ distance from the object of concern, usually the vacuum chamber. The average dose rate along the PS ring is about $250 \mu \mathrm{Sv} / \mathrm{h}$, with $40 \%$ of the measured points below $100 \mu \mathrm{Sv} / \mathrm{h}$ and only $5 \%$ above $1 \mathrm{mSv} / \mathrm{h}$. Simulations with three-month continuous irradiation of ${ }^{6} \mathrm{Li}$ and ${ }^{18} \mathrm{~F}$ and one-week waiting show that the dose rate at $40 \mathrm{~cm}$ distance from a PS magnet would range between $60 \mu \mathrm{Sv} / \mathrm{h}$ and $2 \mathrm{mSvh}$ which is relatively high compared to the present level of induced radioactivity. This indicates that the tunnel would remain accessible with limited stay during maintenance, as long as the maintenance operations are well planned and optimized in order to reduce doses to workers. These values also suggest that there might be magnets whose levels of induced radioactivity require remote handling. This can only be confirmed or ruled out by a detailed study with precise and realistic scenarios of beam losses and with the implementation of the exact material composition (including traces). The effective dose given to the reference population in a one-year operation of the PS, due to the air release in the environment, is $0.37 \mu \mathrm{Sv}$ for the He operation and $0.35 \mu \mathrm{Sv}$ for the $\mathrm{Ne}$ operation. For comparison, the total effective dose given to the population by all CERN installations must not exceed $10 \mu \mathrm{Sv}$.

\section{PS injection issues}

The baseline foresees the injection in the PS at $3.5 \mathrm{GeV}$ proton-equivalent kinetic energy. A batch of 20 bunches, arriving from the RCS with a repetition rate of $10 \mathrm{~Hz}$, are accumulated on a long flat bottom (1.9 s), then accelerated up to $26 \mathrm{GeV}$ proton-equivalent and sent to the CERN-SPS.

\section{Injection energy}

The $3.5 \mathrm{GeV}$ injection energy, to be compared with the CERN operational beams energy of $1.4 \mathrm{GeV}$, has been chosen from space-charge consideration and it is based on a maximum Laslett tune shift of 0.22 [77]. This is a safe margin, as some high intensity operational beams in the PS have a space-charge tune spread larger than 0.3 (although they are immediately accelerated after injection).

Considerations of septum technology, kicker strength and straight-sections length in the PS indicate that injection at $3.5 \mathrm{GeV}$ p-equivalent is challenging, as also confirmed by PS and injection experts [78].

It is therefore here proposed to lower the beta-beams injection energy to $2 \mathrm{GeV}$ p-equivalent. This value corresponds to what is studied for the LHC Injectors Upgrade project and will allow beta beams to profit of synergies with this project and eventually to use the same equipments. 
A recent study of a $10 \mathrm{~Hz}$ RCS as a PS preinjector, to replace the CERN-PS booster [79], proposes the eddycurrent technology for the septum and the bumper, plus an extra kicker in addition to what is already installed in the PS, to provide the $30 \%$ increase in kick strength for the $2 \mathrm{GeV}$ injection energy. This work, which will be the starting point when a detailed study of the beta-beams injection scheme is required, also identifies issues that need careful study in case of multiple bunches injection from the RCS, namely losses due to the injection bump, the lifetime of eddy-current devices and a vertical acceptance reduction at the septum, compared to what the PS can accept nowadays.

\section{Long flat bottom}

The injection flat bottom is $1.9 \mathrm{~s}$ long, since the PS is receiving 20 injections at $10 \mathrm{~Hz}$ from the RCS. This has two consequences. First of all, the decay losses on the long flat bottom induce a $43 \%$ reduction of the ${ }^{6} \mathrm{He}$ intensity and a $18 \%$ for ${ }^{18} \mathrm{Ne}$. Studies [80] of dose deposition in the magnets and FLUKA simulations of losses in "hot" regions identify no show stoppers, as discussed in Chapter VII.

The second issue is represented by collective effects and in particular space charge, which may induce beam blowup and losses on the long injection flat bottom.

\section{Space charge}

Space charge induces tune spread in the beam and can make particles in high-intensity and high-brightness beams cross betatron resonances and either be lost, or contribute to emittance growth. Usually the value of $\left|\Delta Q_{\mathrm{SC}, \max }\right|=0.22$ is assumed for the determination of the maximum allowed Laslett tune shift. This is a safe margin, useful in the design process, but it can be relaxed.

The identification of the destructive resonance lines and the determination of the optimum working point and the maximum Laslett tune shift, via beam-based measurements, is fundamental to fully exploit the PS intensity performances for the beta beams, especially if an injection at a lower energy ( $2 \mathrm{GeV}$ p-equivalent) has to be implemented. For the following measurements, the proton beams available in the PS are used, but the results can be easily scaled to the beta-beams ions.

\section{Tune scan for destructive resonance-lines identification}

The identification of the most dangerous resonances [81] is realized by applying the method proposed in [82]: a large emittance, moderate intensity, not space-charge dominated beam is kept on a $1.2 \mathrm{~s}$ magnetic flat bottom at constant energy of $2 \mathrm{GeV}$. The emittance of the beam is chosen to be large enough to fill at maximum the vacuum chamber, in such a way that even a weak resonance produces a limited increase of the betatronic amplitude for which losses should become visible. During the scan, the tune is slowly varied

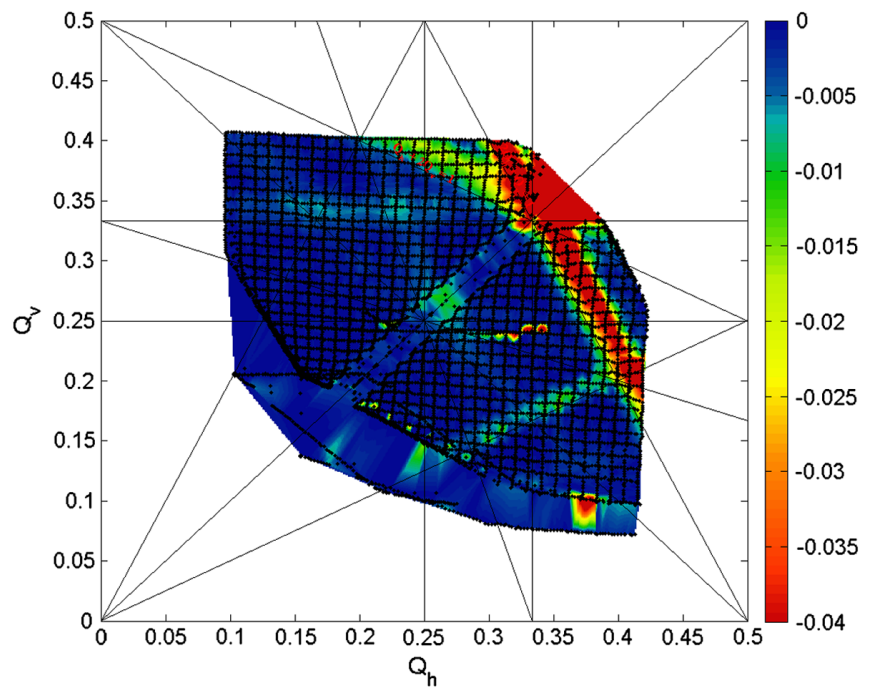

FIG. 34. Tune diagram resulting from the loss measurement at $2 \mathrm{GeV}$. The color scale indicates the derivative of the BCT signal, i.e., the red lines indicate the larger losses.

with a predefined function and the beam intensity is recorded: if an excited resonance is crossed, beam losses are observed as a slope in the beam current transformer (BCT) acquisition.

Figure 34 shows the tune scan for the bare machine (without nonlinear elements such as sextupoles, octupoles or pole face windings). The black dots indicate the points at which the tune and losses were measured. There are many measurements close to each other; on the plot we see measurement where one tune has been held constant and the other has been varied. The color plot is then obtained by a MATLAB (C) interpolation function. The losses are quoted in terms of the derivative of the BCT signal $d N / N$.

The plot identifies the lines from which the working point should be kept far from. It indicates that the most destructive line is the $2 q_{x}+q_{y}=1$ and that the fourth order line $4 q_{y}=1$ is not excited, but the third order $3 q_{y}=1$ is.

\section{Space-charge dominated beam measurements}

Measurements [83] with a space-charge dominated beam (Laslett tune shift was $\Delta Q_{x}=-0.21, \Delta Q_{y}=-0.29$ ) consisted in a scan over the vertical line $Q_{x}=6.20$ in steps of $\delta Q_{y}=0.01$ for vertical tune values in the range $6.21<Q_{y}<6.39$. The beam was kept on the $2 \mathrm{GeV}$ plateau for a fixed working point, the transverse profiles were measured at the beginning and after $1 \mathrm{~s}$, with wire scanners, and the longitudinal profile evolution and intensity recorded over the entire plateau.

The main result is the identification of a range of tunes between $6.21<Q_{y}<6.26, Q_{x}=6.20$ for which no losses and no profile degradation occur. This represents a region in the working-point space in which also the ${ }^{6} \mathrm{He}$ beam $\left(\Delta Q_{x}, \Delta Q_{y}\right)=(-0.22,-0.31)$ should survive. For the 
${ }^{18} \mathrm{Ne}$ beam $\left(\Delta Q_{x}, \Delta Q_{y}\right)=(-0.28,-0.38)$, instead, more work is needed (chromaticity correction and/or resonance compensation).

\section{THE CERN SPS}

\section{A. The rf of the SPS}

Studies of the beta beam in the SPS within the EURISOL Design Study (see [12] for more information and references) will be briefly summarized here for completeness. Rather than consider a new machine, the space-charge bottleneck at SPS injection has been addressed by adding a "modest" $40 \mathrm{MHz}$ rf system to the existing infrastructure. This would allow much longer bunches to be transferred from the PS, the matching voltage for $20 \mathrm{~ns}$ bunches being about 120 and $5 \mathrm{kV}$ for helium and neon, respectively. Near transition when the bunches are short enough, the standard $200 \mathrm{MHz}$ system of the SPS would take over. However, buckets have very different aspect ratio which means that mismatch of the bunches is unavoidable.

$1 \mathrm{MV}$ at $40 \mathrm{MHz}$ is at the limit of what might be considered as modest and constrains the maximum ramp rate to around $0.1 \mathrm{~T} / \mathrm{s}$ in the early part of the cycle. Even so, the ramp rate must be slowed down still further for the rebucketing because even a small ramp rate reduces the $200 \mathrm{MHz}$ bucket length and buying this back with voltage is costly in terms of mismatch. Assuming a ramp rate of $0.02 \mathrm{~T} / \mathrm{s}$ and that the emittance the SPS is supposed to deliver is already established before transition, proximity to transition $\left(\gamma_{t r}=23\right)$ reduces the $200 \mathrm{MHz}$ voltage that is required to accommodate the bunch length accelerated in the proposed new $40 \mathrm{MHz}$ bucket. Performing rf gymnastics close to transition is bound to be a delicate matter, but it incurs no penalty in mismatch because the aspect ratios of the two buckets scale identically with Lorentz $\gamma$.

Despite a larger emittance, the situation is easier in the neon case due to its advantageous charge-to-mass ratio. Although rebucketing must still be performed at the same small ramp rate of $0.02 \mathrm{~T} / \mathrm{s}$, proximity to transition can be decreased to $\gamma=20$ and still have $1 \mathrm{MV}$ at $40 \mathrm{MHz}$ changing over to $1.75 \mathrm{MV}$ at $200 \mathrm{MHz}$. 7.8 $\mathrm{MV}$ at $200 \mathrm{MHz}$ is needed to rematch. $1 \mathrm{MV}$ is the minimum $40 \mathrm{MHz}$ voltage requires. It also costs cycle time because of the need to slow the ramp rate down to permit rebucketing. However, since the $40 \mathrm{MHz}$ system sees almost all the frequency swing during acceleration, more voltage would be expensive. Alternatively, one could consider rebucketing at zero ramp rate as this reduces slightly the problem of matching. The longitudinal emittance that the SPS must deliver is $1.0 \mathrm{eV} \mathrm{s}$ in the case of helium ions and $2.2 \mathrm{eV}$ s for neon. These values are derived from the known performance for protons and, allowing an emittance budget of some $25 \%$ for blowup during each acceleration stage, they also fix those in all the upstream machines. The injection scheme proposed for the decay ring requires the beam to be delivered off momentum into the nonlinear region of the receiving bucket. Consequently, the bunch is deliberately mismatched before extraction from the SPS by a step down in $200 \mathrm{MHz}$ voltage. This bunch tilting is a first-order attempt to increase the capture efficiency at the end of a quarter of a synchrotron turn in the decay ring. The fine detail of capture will depend on the large-amplitude distribution created in the SPS.

\section{B. Vacuum system requirements for the SPS}

The losses in the SPS are dominated by radioactive decay. Losses due to charge exchange are negligible, as the cross sections for electron capture are very small. For both ${ }^{6} \mathrm{He}^{2+}$ and ${ }^{18} \mathrm{Ne}^{10+}$ the losses are peaked behind the quadrupole magnets. The transmission was checked by using the proposed cycle assuming the maximum number of particles coming from the PS. The resulting number of particles ejected from the SPS are arguably identical with the desired numbers of $9.0 \times 10^{12}$ and $4.26 \times 10^{12}$ for ${ }^{6} \mathrm{He}^{2+}$ and ${ }^{18} \mathrm{Ne}^{10+}$ respectively.

Every SPS magnet has an ion pump with a pumping speed of $20 \mathrm{l} / \mathrm{s}$. Considering the main magnets only, a conductance corrected effective pumping speed of $S_{\text {eff }}=$ $2.6 \mathrm{~m}^{3} / \mathrm{s}$ was calculated. Placing pumps at the positions where there are no dipoles in the ring leads to $S_{\text {eff }}=2.8 \mathrm{~m}^{3} / \mathrm{s}$. All simulations carried out for the SPS assume $S_{\text {eff }}=2.8 \mathrm{~m}^{3} / \mathrm{s}$.

Ionization of residual gas particles by the revolving beam, called target ionization, is the dominant effect that causes a pressure rise in the SPS during beta-beam operation. The ionized gas particles are accelerated away from the beam by its space-charge potential. When these particles hit the vacuum chamber, a low energy desorption process takes place. The desorption rate, $\eta$, for this process is considered to be in the range between 1 and 10 desorbed particles per ionized gas particle hitting the vacuum chamber. The pressure evolution strongly depends on the assumed desorption rate. For ${ }^{6} \mathrm{He}^{2+}$ the residual gas pressure is not stable when a desorption rate greater than 5 is assumed, while for ${ }^{18} \mathrm{Ne}^{10+}$ even in case of $\eta=1$ the pumping speed is not sufficient to stabilize the pressure.

One possibility to reduce the pressure buildup is to reduce the acceleration time within the SPS. The acceleration time can be shortened from 2.54 to $1.58 \mathrm{~s}_{\text {for }}{ }^{6} \mathrm{He}^{2+}$ and from 1.42 to $0.90 \mathrm{~s}$ for ${ }^{18} \mathrm{Ne}^{10+}$, when using the maximal available ramping rate of the SPS of $0.74 \mathrm{~T} / \mathrm{s}$. Furthermore the cycle time of the ${ }^{18} \mathrm{Ne}^{10+}$ cycle was extended to $6 \mathrm{~s}$ in order to give the vacuum more time to relax. This would slow down the pressure rise for ${ }^{6} \mathrm{He}^{2+}$ operation with $\eta=10$ and stabilize the ${ }^{18} \mathrm{Ne}^{10+}$ operation with $\eta=1$. In this scenario accelerating ${ }^{18} \mathrm{Ne}^{10+}$ ions with $\eta>1$ is still not feasible.

As shown before the operation with ${ }^{6} \mathrm{He}^{2+}$ is stable for $\eta=5$. At a pumping speed of $4 \mathrm{~m}^{3} / \mathrm{s}$ the pressure is 
stabilized below $1 \times 10^{-8}$ mbar. For ${ }^{18} \mathrm{Ne}^{10+}$ various combinations of $\eta$ and $S_{\text {eff }}$ have been calculated assuming the maximal ramping rate and an extended cycle time of $6 \mathrm{~s}$. For each $\eta=1,3$ and 5 the required pumping speeds were estimated to be $2.8 \mathrm{~m}^{3} / \mathrm{s}, 7.5 \mathrm{~m}^{3} / \mathrm{s}$ and $12.0 \mathrm{~m}^{3} / \mathrm{s}$ respectively. Simulations show that during SPS operation with ${ }^{6} \mathrm{He}^{2+}$ or ${ }^{18} \mathrm{Ne}^{10+}$, there is a massive pressure buildup due to ionization of residual gas particles induced by the circulating beam. It could be shown that an operation with ${ }^{6} \mathrm{He}^{2+}$ ions is possible, if the desorption rate for ionized gas particles hitting the vacuum chamber is less or equal to 5 . In this case the residual gas pressure stays below $1 \times 10^{-8}$. Should the desorption rate be greater than 5 , the pressure can be stabilized by reducing the acceleration time from 2.54 to $1.58 \mathrm{~s}$ by using the maximal available ramping rate of $0.74 \mathrm{~T} / \mathrm{s}$ and increasing the total effective pumping speed to approximately $4 \mathrm{~m}^{3} / \mathrm{s}$. An operation using ${ }^{18} \mathrm{Ne}^{10+}$ ions with the proposed cycle is not possible without adjusting the cycle or the pumping speed. In case of a desorption rate $\eta=1$ either the effective pumping speed has to be increased to about $7.5 \mathrm{~m}^{3} / \mathrm{s}$ or the acceleration time has to be minimized by using the maximal available ramping rate of the SPS, while the cycle has to be extended to $6 \mathrm{~s}$ in order to give the vacuum enough time to relax. The increased ramping rate reduces the acceleration time from 1.42 to $0.90 \mathrm{~s}$. If the desorption rate is greater than 1 , the higher ramping rate and the extended cycle of $6 \mathrm{~s}$ have to be combined with a higher effective pumping speed. For desorption rates $\eta=1$, 3 and 5 effective pumping speeds of $2.8 \mathrm{~m}^{3} / \mathrm{s}, 7.5 \mathrm{~m}^{3} / \mathrm{s}$ and $12.0 \mathrm{~m}^{3} / \mathrm{s}$ have to be applied.

Losses occurring in the PS and the SPS for the beta-beam operation are in the same order of magnitude as CNGS for nominal intensities of ${ }^{6} \mathrm{He}$ and ${ }^{18} \mathrm{Ne}$ and are therefore not a show stopper for the project.

The studies of the PS and the SPS have to be reconsidered in the case the cycling and the rf needs modification to get better conditions for the overall beta beam in the CERN accelerators: recent measurement values of the oscillation angle $\theta_{13}$ may permit relaxation bunching constraints in the decay ring, which may give more flexibility in the preceding machines.

\section{THE DECAY RING}

The decay ring parameters are summed up in Table VIII.

TABLE VIII. Beam parameters.

\begin{tabular}{|c|c|c|c|c|c|}
\hline & Units & ${ }^{6} \mathrm{He}^{2+}$ & ${ }^{18} \mathrm{Ne}^{10+}$ & ${ }^{8} \mathrm{Li}^{3+}$ & ${ }^{8} \mathrm{~B}^{5+}$ \\
\hline Atomic mass $A_{\text {eff }}$ & $\mathrm{u}$ & 6.019 & 18.006 & 8.022 & 8.025 \\
\hline$E_{\text {rest }} /$ ion & $\mathrm{GeV}$ & 5.606 & 16.772 & 7.471 & 7.473 \\
\hline$\gamma$ & $\ldots$ & 100 & 100 & 100 & 100 \\
\hline$\beta$ & $\cdots$ & 1.00 & 1.00 & 1.00 & 1.00 \\
\hline$\beta \cdot \gamma$ & $\cdots$ & 99.995 & 99.995 & 99.995 & 99.995 \\
\hline Half-life at rest $\tau$ & $\mathrm{s}$ & 0.807 & 0.167 & 0.840 & 0.770 \\
\hline$B \rho$ & $\mathrm{T} \mathrm{m}$ & 934.87 & 559.27 & 830.64 & 498.50 \\
\hline Ring length & $\mathrm{m}$ & 6911.5 & 6911.5 & 6911.5 & 6911.5 \\
\hline Revolution time & $\mu \mathrm{s}$ & 23.06 & 23.06 & 23.06 & 23.06 \\
\hline Number of bunches & $\ldots$ & 20 & 20 & 20 & 20 \\
\hline Normalized $\epsilon_{x}(1 \sigma)$ & $\pi \mathrm{mm} \mathrm{mrad}$ & 14.8 & 14.8 & & \\
\hline Normalized $\epsilon_{z}(1 \sigma)$ & $\pi \mathrm{mm} \mathrm{mrad}$ & 7.9 & 7.9 & & \\
\hline Injection cycle time & $\mathrm{s}$ & 6.0 & 3.6 & 4.8 & 3.6 \\
\hline Nominal annual $\nu$ flux & $10^{18}$ & 2.9 & 1.1 & 14.5 & 5.5 \\
\hline \multicolumn{6}{|c|}{ Stored beam } \\
\hline Number of stored ions & $10^{13}$ & 9.346 & 7.178 & 48.18 & 16.70 \\
\hline Number of ions/bunch & $10^{12}$ & 4.673 & 3.589 & 24.09 & 8.35 \\
\hline Full energy of the beam & MJ & 8.3937 & 19.282 & 57.668 & 19.984 \\
\hline Average beam current & A & 1.30 & 4.99 & 10.04 & 5.80 \\
\hline Peak beam current & A & 227.9 & 875.0 & 1762 & 1017 \\
\hline Longitudinal emittance (full) & $\mathrm{eV} \mathrm{s}$ & 14.4 & 43.3 & 19.3 & 19.3 \\
\hline Bunch length & $\mathrm{m}$ & 1.97 & 1.97 & 1.97 & 1.97 \\
\hline Momentum spread (full) & $10^{-3}$ & 2.5 & 2.5 & 2.5 & 2.5 \\
\hline \multicolumn{6}{|c|}{ Injected beam } \\
\hline Relative energy difference & $10^{-3}$ & 5 & 5 & 5 & 5 \\
\hline Number of ions/bunch & $10^{11}$ & 5.57 & 2.70 & 27.6 & 9.17 \\
\hline Full energy of the beam & MJ & 0.475 & 2.99 & 6.61 & 2.20 \\
\hline Longitudinal emittance (full) & $\mathrm{eV} \mathrm{s}$ & 1.0 & 2.2 & 1.33 & 1.33 \\
\hline Bunch length & $\mathrm{m}$ & 1.197 & 1.197 & 1.197 & 1.197 \\
\hline Momentum spread (full) & $10^{-3}$ & 0.4 & 0.4 & 0.4 & 0.4 \\
\hline
\end{tabular}


After presenting the latest version of beta-beam decay ring (DR), the scheme to inject and accumulate the ions and the losses which occur in the decay ring will be discussed. The required rf system to handle the very large peak intensities in the decay ring bunches that are needed to have sufficient signal/noise ratios in the detectors has been designed. The decay ring has been optimized to permit a maximum number of ions to be stored in the decay ring; studies made will be described.

\section{A. Optics}

\section{First order optics of the DR}

The circumference of the decay ring was chosen to be the same as for the SPS (6911.5 m) to keep the same temporal structure at the injection. The decay ring is racetrack shaped to permit neutrinos to obtain a $\gamma$ boost in the direction of the detector. The long straight sections must be as long as possible to maximize the neutrino flux towards the detector. The length of the long straight section is equal to $2572 \mathrm{~m}$, which corresponds to $37.2 \%$ of the total length and the length of the arcs is then $876 \mathrm{~m}$ with a compaction of about $50 \%$. The dipoles are $7 \mathrm{~m}$ long with an angle of $\pi / 70 \mathrm{rad}$, which corresponds to a curvature radius of $156 \mathrm{~m}$ and a magnetic field of $6 \mathrm{~T}$. Five functional parts can be distinguished in the ring [84-86]: (i) a long straight section which is directed to the detector, (ii) regular FODO lattices in the arcs, (iii) dispersion suppressors at the arc boundsthey are used to extract the decay products coming from the straight section, (iv) a collimation section in energy, (v) an insertion for the injection.

Improvements of the lattice $[87,88]$ have been made within EUROnu to locate the injection in a chicane in the straight section which is not directed to the detector [89]. Moreover, the momentum collimation is located in the chicane which makes it possible to have only FODO lattices in the arcs. Another advantage of the chicane is to enlarge the momentum compaction of the ring, which relaxed the head tail instabilities, as explained in the subsection about the collective effects (see Sec. IXF). The drawback of this solution is to require extra dipoles for the injection and to increase the needed total $\mathrm{rf}$ voltage because of a larger slip factor.

In order to keep a large dynamic aperture, the arcs are symmetric and are realized as $2 \pi$ insertions. The working point of the ring is then determined by the optics of the long straight sections. The working point, $Q_{x}=18.228$ and $Q_{z}=18.16$, was chosen far from the second and third order resonances.

The periodicity of the decay ring is 1 because of the chicane in one of the long straight sections. A schematic layout of the decay ring is given in Fig. 35.

The optical functions of the decay ring are given in Fig. 36 at the reference energy and at the injection energy $(\delta=5 \%)$. The stored beam will be assumed to be collimated in energy at $\delta_{C}=2.5 \%$. The beam sizes at $6(5)$

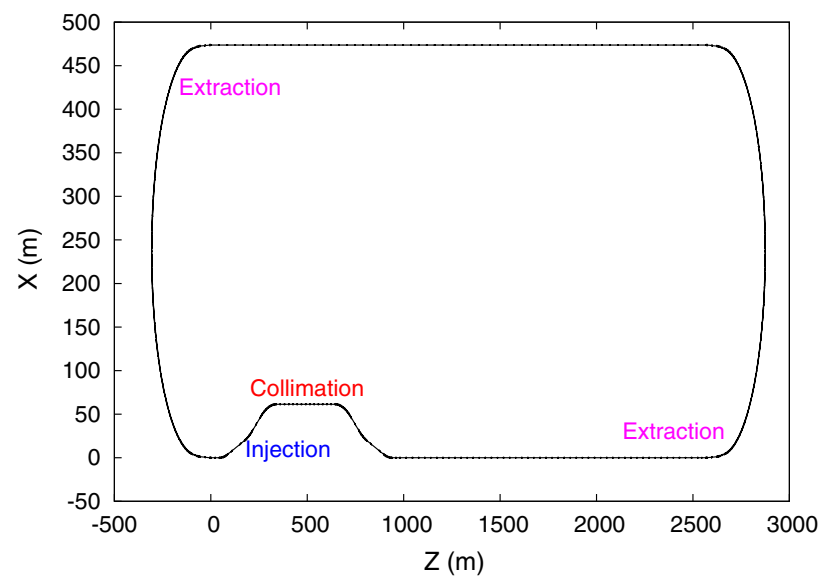

FIG. 35. Layout of the beta-beam decay ring.

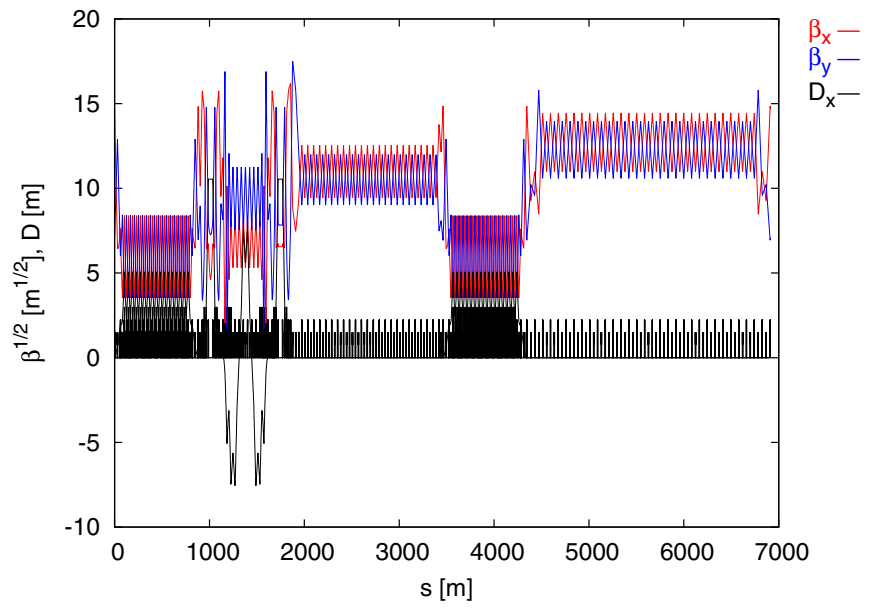

(a) $\delta=0$

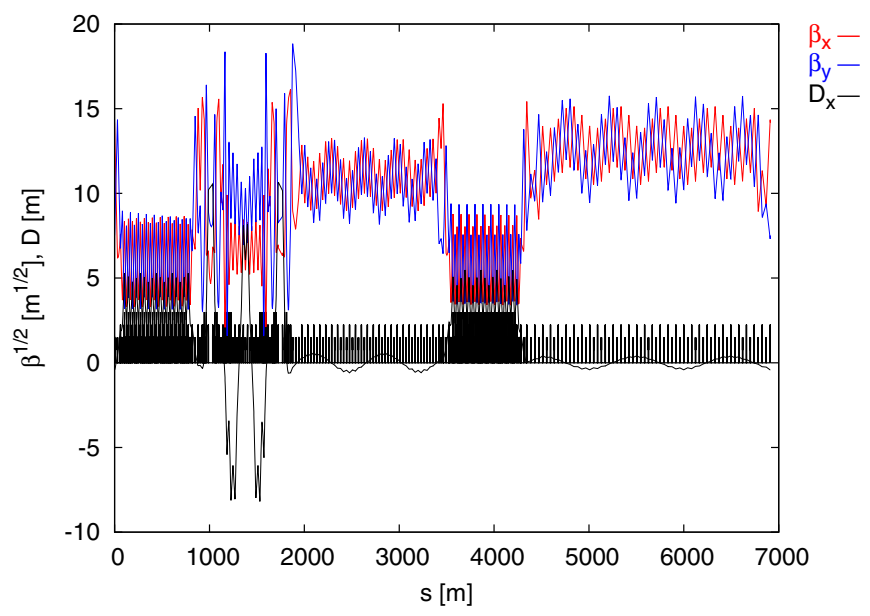

(b) $\delta=5 \%$ o

FIG. 36. Optical functions in the beta-beam decay ring at the reference energy and at the injection energy. 


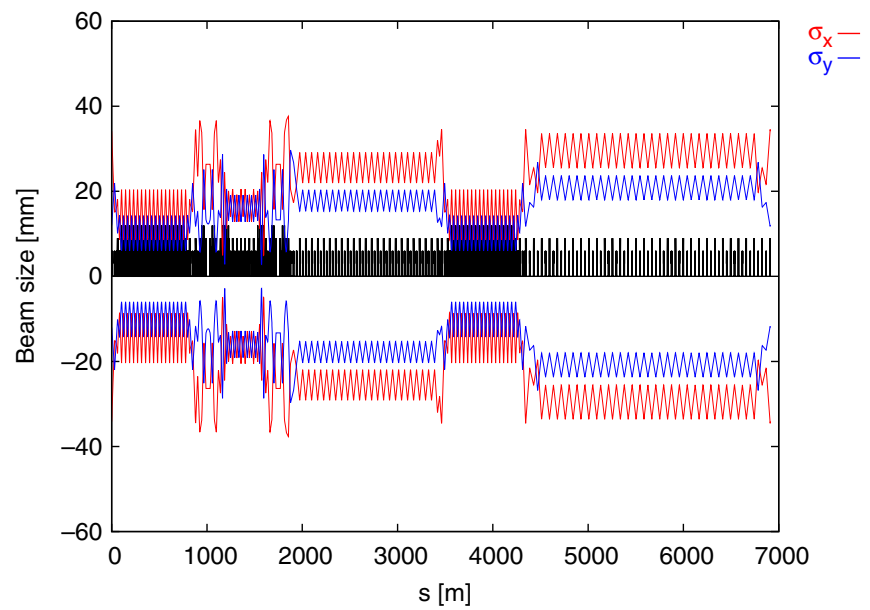

(a) $\delta=0$

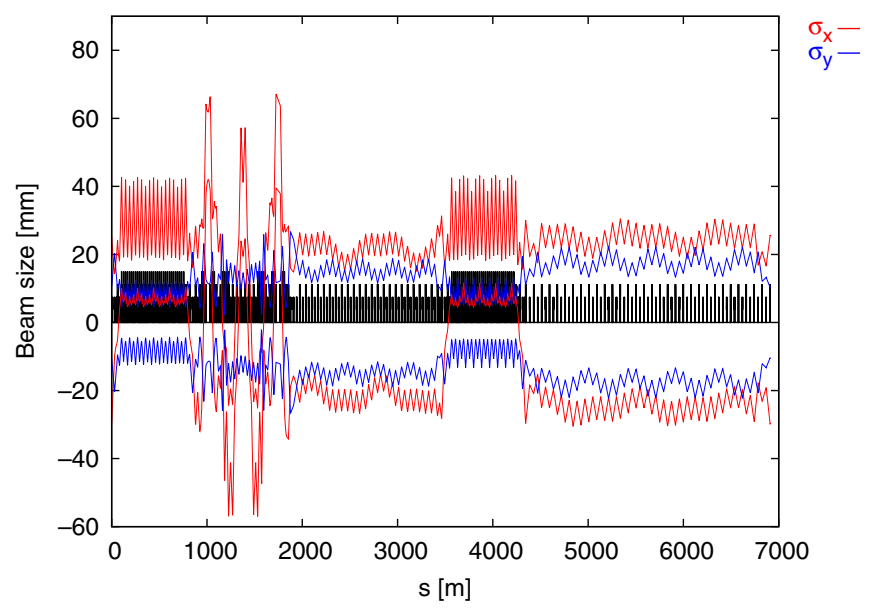

(b) $\delta=5 \%$

FIG. 37. Beam sizes at $6 \sigma$ and $5 \sigma$ in the decay ring respectively at the reference (top) and injection energy (bottom).

standard deviations for the stored (injected) beam are given in Fig. 37 for the injected and stored beams. The global parameters of the decay ring are summed up in Table IX. The parameters at the injection point into the decay ring are summed up in Table X. Except near the extraction and injection points, a half aperture of $60 \mathrm{~mm}$ is sufficient in all elements, as Fig. 37 shows. Most superconducting magnets would preferably have coil-free midplanes to prevent magnet quenching due to high energy deposition from decay products impinging the superconducting coils [90]. Studies with thick stainless steel liners to protect the magnets (similar to the LHC insertion quadrupoles) indicate that reasonably thick liners would probably not be sufficient to fully protect the coils, but could be further investigated for other equipment protection [91]. The operational dipole field is $6 \mathrm{~T}$ and the maximum gradient for the quadrupoles should be $42 \mathrm{~T} / \mathrm{m}$ for such apertures [92]. Special care was taken to keep the gradients of the
TABLE IX. Parameters of the decay ring.

\begin{tabular}{lcc}
\hline \hline Length & $\mathrm{m}$ & 6911.5 \\
\hline Machine radius & $\mathrm{m}$ & 1100 \\
$\alpha$ & $10^{-3}$ & 3.555 \\
$\gamma_{\mathrm{tr}}$ & $\ldots$ & 16.772 \\
$Q_{x}$ & $\ldots$ & 18.228 \\
$Q_{y}$ & $\ldots$ & 18.160 \\
$Q_{\mathrm{x}, \text { nat }}^{\prime}$ & $\ldots$ & -22.871 \\
$Q_{\mathrm{y}, \text { nat }}$ & $\ldots$ & -25.867 \\
$\beta_{\mathrm{x}, \text { max }}$ & $\mathrm{m}$ & 262.750 \\
$\beta_{\mathrm{y}, \text { max }}$ & $\mathrm{m}$ & 306.123 \\
$D_{\mathrm{x}, \max }$ & $\mathrm{m}$ & 10.544 \\
Maximum dipole field & $\mathrm{T}$ & 5.984 \\
Number of dipoles & $\ldots$ & 176 \\
Maximum quadrupole gradient & $\mathrm{T} / \mathrm{m}$ & 36.049 \\
Number of quadrupoles & $\ldots$ & 235 \\
\hline \hline
\end{tabular}

quadrupoles as low as possible; they are less than $35 \mathrm{~T} / \mathrm{m}$ (Fig. 38).

\section{Dynamic aperture}

The natural chromaticity in the decay ring is $Q_{x}^{\prime}=$ -22.871 (or $Q_{x}^{\prime} / Q_{x}=-1.255$ ) and $Q_{y}^{\prime}=-25.867$ (or $\left.Q_{y}^{\prime} / Q_{y}=-1.424\right)$. In order to accept the injected beam at $\delta=5 \%$, the natural chromaticity must be corrected by sextupole families in the dispersive areas. Two sextupole families located in the arcs are used. The phase advance per FODO lattice in the arcs is $\pi / 2$ in both planes, which enables to compensate some geometric aberrations due to the presence of sextupoles. The dynamic aperture for 10 000 turns at the center of the chicane in the energy range of the stored beam can be seen in Fig. 39. The rms beam size in the horizontal and vertical planes is respectively $1.782 \mathrm{~mm}$ and $2.054 \mathrm{~mm}$. The dynamic aperture is large enough to accept the whole beam (more than 20 $\sigma$ ).

TABLE X. Optical parameters at the injection point.

\begin{tabular}{llcc}
\hline \hline & \multicolumn{1}{c}{ Units } & Stored beam & Injected beam \\
\hline$\beta_{x}$ & $\mathrm{~m}$ & 25.0 & 26.1 \\
$\beta_{z}$ & $\mathrm{~m}$ & 54.1 & 64.2 \\
$\alpha_{x}$ & $\cdots$ & -0.43 & -0.45 \\
$\alpha_{z}$ & $\cdots$ & -0.17 & -0.02 \\
$D_{m}$ & $\mathrm{~m}$ & 10.54 & 10.46 \\
$\Delta E / E_{0}$ & $10^{-3}$ & 0 & 5 \\
$\sigma_{E} / E_{0}$ & $10^{-3}$ & 2.5 & 0.4 \\
$\epsilon_{x}$ & $\pi \mathrm{mm} \mathrm{mrad}$ & 0.15 & 0.15 \\
$\epsilon_{z}$ & $\pi \mathrm{mm} \mathrm{mrad}$ & 0.08 & 0.08 \\
$n$ & $\cdots$ & 6 & 5 \\
Beam size & $\mathrm{mm}$ & 26.4 & 10.7 \\
$e_{S}$ & $\mathrm{~mm}$ & \multicolumn{3}{c}{36.5} \\
$X_{\mathrm{CO}}$ & $\mathrm{mm}$ & \multicolumn{3}{c}{} \\
\hline \hline
\end{tabular}




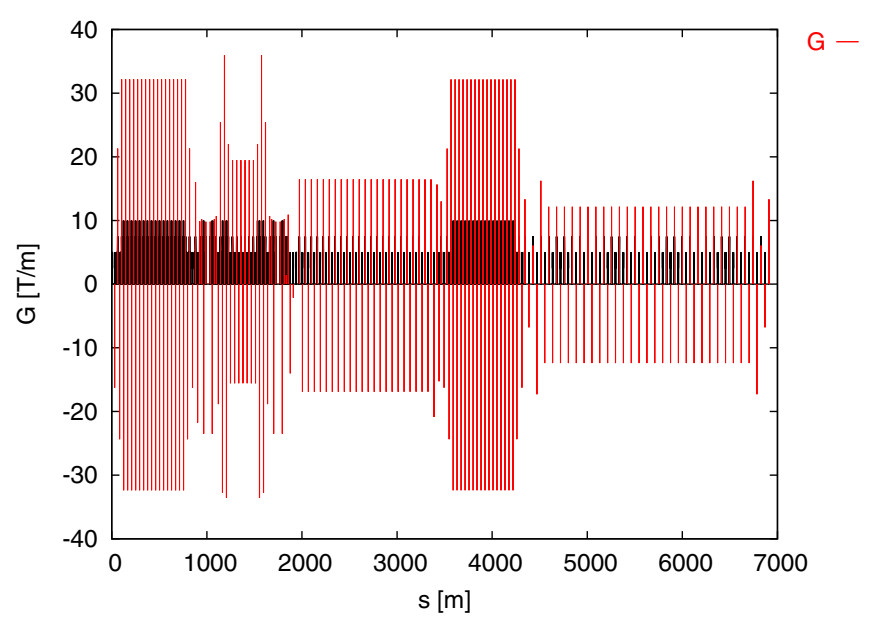

FIG. 38. Gradients of the quadrupoles in the beta-beam decay ring.

\section{B. Correction of the closed orbit}

Unavoidable magnet misalignments and errors of the main magnetic field occur in the magnetic elements in the arcs. One of the consequences is a distortion of the closed orbit $[93,94]$. The code BETA [95] enables one to calculate the rms closed orbit in the presence of these defects. The assumed tolerances for the misalignments are given in Table XI according to the values for LHC [96] and RHIC [97]. The standard deviation of the residual closed orbit without correction is then given in Fig. 40. The beam position monitors (BPMs) are assumed to be ideal. It appears that the rms error on the closed orbit is a few centimeters, which makes the closed orbit correction necessary. 120/117 horizontal/vertical dipole correctors were inserted near focusing/defocusing quadrupoles in the whole structure and 120/117 horizontal/vertical BPMs were needed. After correction, the rms error of the closed orbit is less then $0.7 \mathrm{~mm}$ (see Fig. 40). The maximum of the distortion is in the chicane; no corrector

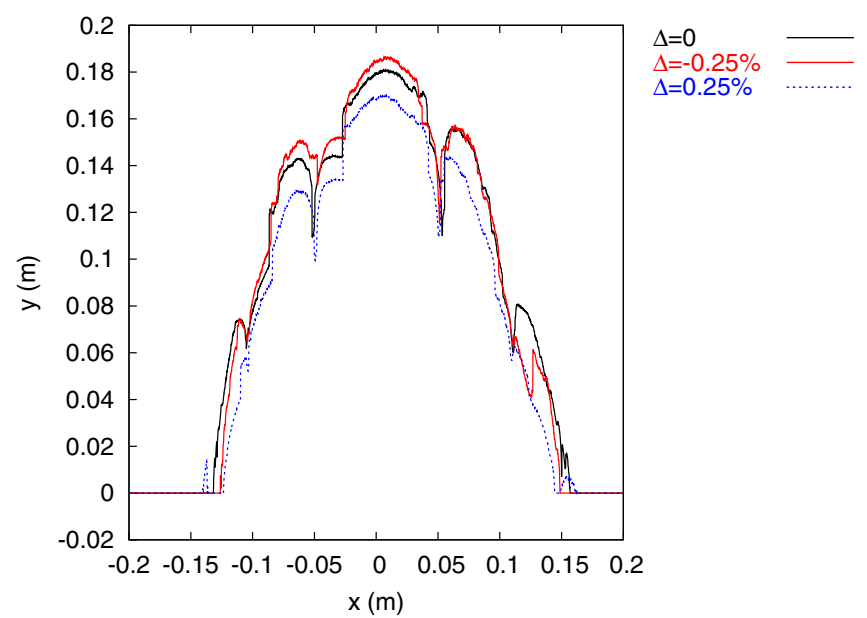

FIG. 39. Dynamic aperture around the chromatic orbit for 10,000 turns at the middle of the injection insertion.
TABLE XI. Assumed tolerances for magnetic elements.

\begin{tabular}{lcc}
\hline \hline Defect type & Units & rms value \\
\hline \multicolumn{3}{c}{ Dipoles } \\
\hline$\underline{\Delta B}$ & $10^{-3}$ & \\
Horizontal misalignment & $\mathrm{mm}$ & 0.5 \\
Vertical misalignment & $\mathrm{mm}$ & 0.5 \\
Longitudinal misalignment & $\mathrm{mm}$ & 0.5 \\
Rolling error & $\mathrm{mrad}$ & 0.5 \\
& Quadrupoles & 1 \\
$\frac{\Delta k}{k}$ & $10^{-3}$ & \\
Horizontal misalignment & $\mathrm{mm}$ & 0.4 \\
Vertical misalignment & $\mathrm{mm}$ & 0.4 \\
\hline \hline
\end{tabular}

near the injection point was inserted. The angular distortion of the closed orbit in the long straight section is less than $0.01 \mathrm{mrad}$ and very small compared to $1 / \gamma$. The contribution to the divergence of the neutrino flux is then negligible. The maximum rms value is respectively

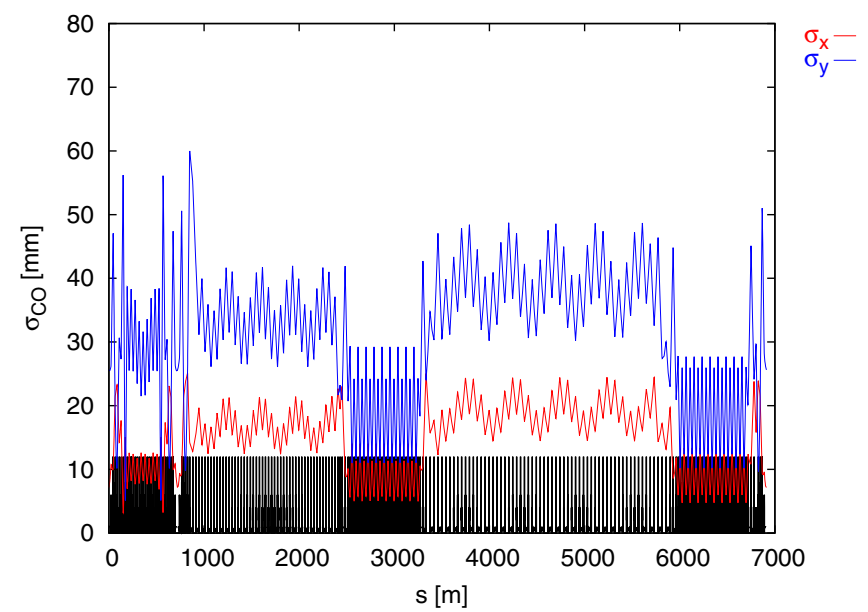

(a) Correction off

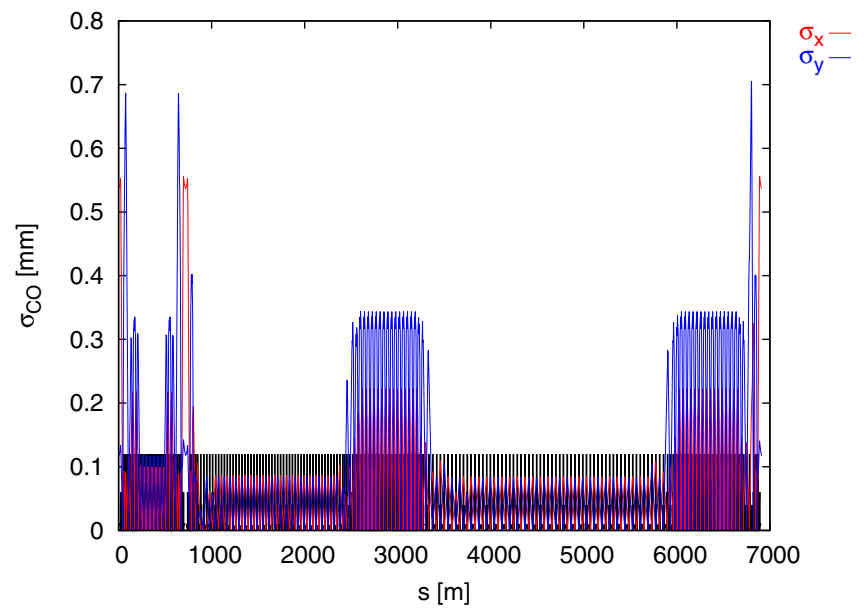

(b) Correction on

FIG. 40. Closed orbit distortion (rms value) without and with correction (in red, horizontal distortion and in blue vertical one). 
$0.046 \mathrm{mrad}$ for a horizontal dipole corrector and $0.070 \mathrm{mrad}$ for a vertical dipole corrector. It is assumed that the closed orbit can be corrected up to 3 standard deviations. Finally, the integrated field in the dipole correctors must be respectively $0.128 \mathrm{~T} \mathrm{~m}$ and $0.195 \mathrm{~T} \mathrm{~m}$.

\section{Case of a decay ring at $\gamma=350$}

New scenarios for the beta beams were proposed by the EUROnu physics work package [98] to increase the physics reach of the beta beam facility. One of the proposals is to accelerate the helium and neon ions to higher $\gamma=350$; some accelerator aspects of this option were investigated. The required neutrino flux is kept the same (this is compatible with estimated possible production rates): $2.9 \times 10^{18}$ antineutrinos per year from the decay of ${ }^{6} \mathrm{He}^{2+}$ and $1.1 \times 10^{18}$ neutrinos per year from the decay of ${ }^{18} \mathrm{Ne}^{10+}$. Figure 41 shows an overview of the impact of different solutions for having higher neutrino energies. We see in this figure the practical and technological challenges and the interest to choose a gamma factor with care. Magnets of over $20 \mathrm{~T}$ are today not possible to realize.

Presently, the highest $\gamma$ value that can be reached with the SPS is 450 for protons, which means a $\gamma=150$ for helium (by scaling with Z/A). Injecting ions at $\gamma=350$ implies that the SPS has to be upgraded to higher energies.

Estimations were made to quantify some of the implications of a higher $\gamma$ decay ring, and to compare with the $\gamma=100$ case and with the LHC [99]. For a decay ring at $\gamma=350$, the acceleration scheme has not been studied. In order to make a comparison, we have made some

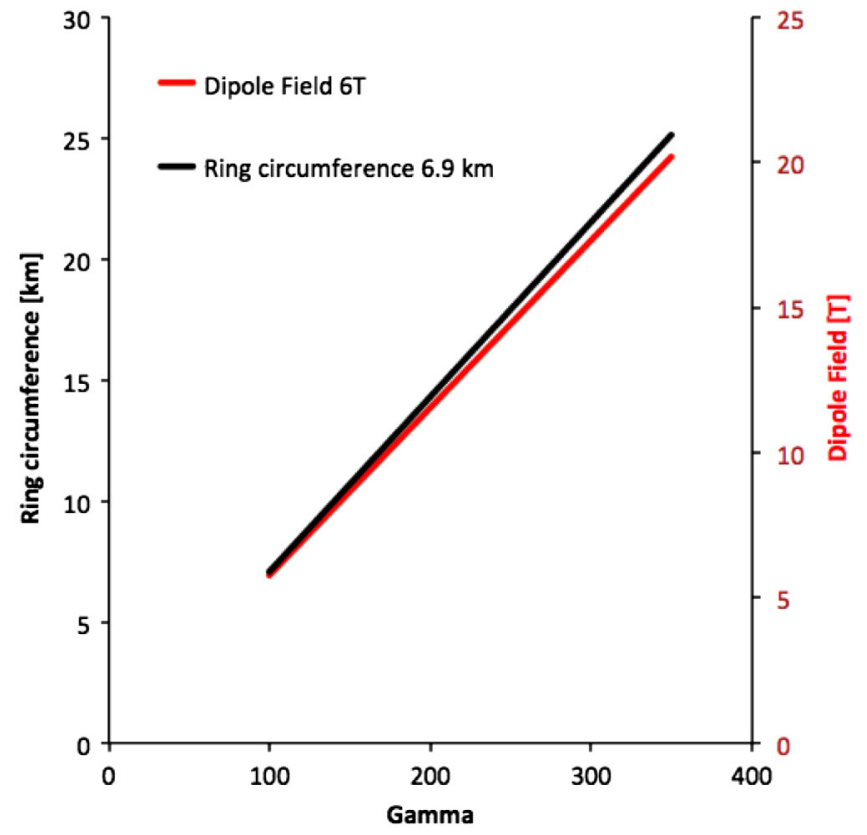

FIG. 41. Dependence of the gamma factor on ring circumference (constant field, $6 \mathrm{~T}$ ) and of the field (constant ring circumference, $6.9 \mathrm{~km})$. assumptions: (i) The ramping speed of the upgraded SPS is the same as the SPS. Therefore, more time is needed to reach $\gamma=350$ for the ions, which implies an increase the repetition time $T_{\text {rep }}$. (ii) The normalized transverse emittance is the same as for the $\gamma=100$ case. The acceleration scheme before injecting into the upgraded SPS is assumed to be the same. (iii) The rf system of the decay ring is not changed; it is assumed that the same voltage and the same rf frequency are kept. (iv) The bunch length is not changed. The longitudinal emittance of the beam is then deduced from this constraint. (v) The merging is assumed to occur without any errors and with the same rf program. In other terms, the number of merges $n_{\text {merges }}$ before losing the ions is the ratio between the longitudinal emittance of the stored beam and the one of the incoming beam. The longitudinal emittance of the incoming beam is assumed to be the same as in FP6. The number of stored ions $N_{\text {tot }}$ can be deduced from the number of injected ions $N_{\text {inj }}$ by

$$
N_{\text {tot }}=N_{\text {inj }} \frac{1-2^{-\frac{n_{\text {merges }} T_{\text {Tep }}}{\gamma \tau}}}{1-2^{-\frac{T_{\text {rep }}}{\gamma \tau}}} .
$$

(vi) The theoretical single bunch intensity limit from collective effects due to transversal mode coupling is calculated from the formula [see Eq. (24)] [100]:

$$
N_{b x, y}^{\mathrm{th}}=\frac{32}{3 \sqrt{2} \pi} \frac{R|\eta| \epsilon_{l}^{2 \sigma} \omega_{r}}{\langle\beta\rangle_{x, y} Z^{2} \beta^{2} c R_{\perp}},
$$

where $R$ is the average ring radius, $\eta$ the slip factor, $\epsilon_{l}^{2 \sigma}$ the longitudinal emittance at $2 \sigma$ in eV s, $\omega_{r}$ the cut pulsation linked to the beam pipe radius $(6 \mathrm{~cm})$ and $R_{\perp}$ is the transverse wall impedance (assumed to be $1 \mathrm{M} \Omega / \mathrm{m}$ for the decay ring). In reality, that is an optimistic upper limit and generally, the instabilities are excited for a smaller beam intensity. For the LHC, the given impedance is without any collimator and for two frequencies $(8 \mathrm{kHz}$ and $20 \mathrm{MHz})$.

The reference case (noted "ref") at $\gamma=100$ is compared to two scenarios at $\gamma=350$. In the first case (noted "1"), the circumference is kept the same and the field of the dipoles is scaled with the magnetic rigidity. In the second case (noted "2"), the circumference is scaled to keep the same magnetic field in the magnets. The results are summarized in Table XII. In order to make the comparison easier, we have added in the last columns the parameters of the proton beam in the LHC after acceleration.

In the first scenario, the required magnetic fields are high. The technical feasibility of such magnets has to be studied. In the R\&D program for the LHC, an ultimate upgrade would be to double the energy of the protons (DLHC). The magnetic field of the dipoles for the DLHC is about $20 \mathrm{~T}$, which is outstanding. Other challenging items are the stored beam energy, which is about the same as in the LHC and the collective effects. Higher intensities have 
TABLE XII. Decay ring at $\gamma=100, \gamma=350$ high and low dipole field and LHC for protons.

\begin{tabular}{|c|c|c|c|c|c|c|}
\hline & Units & $\mathrm{He}$ & $\mathrm{Ne}$ & $\mathrm{Li}$ & B & LHC $p$ \\
\hline \multirow[t]{2}{*}{ Nominal annual $\nu$ flux } & $10^{18}$ & 2.9 & 1.1 & 14.5 & 5.5 & $\ldots$ \\
\hline & & 100 & 100 & 100 & 100 & 7460.523 \\
\hline \multirow[t]{3}{*}{$\gamma$} & & 350 & 350 & 350 & 350 & 7460.523 \\
\hline & & 350 & 350 & 350 & 350 & 7460.523 \\
\hline & & 81.0 & 167.0 & 83.8 & 77.0 & $\infty$ \\
\hline \multirow[t]{3}{*}{ Half-life time } & s & 283.5 & 584.5 & 293.3 & 269.5 & $\infty$ \\
\hline & & 283.5 & 584.5 & 293.3 & 269.5 & $\infty$ \\
\hline & & 934.87 & 559.27 & 830.64 & 498.50 & 23349 \\
\hline \multirow[t]{3}{*}{ Magnetic rigidity } & $\mathrm{T} \mathrm{m}$ & 3272.2 & 1957.5 & 2907.4 & 1744.8 & 23349 \\
\hline & & 3272.2 & 1957.5 & 2907.4 & 1744.8 & 23349 \\
\hline & & 6911.5 & 6911.5 & 6911.5 & 6911.5 & 26659 \\
\hline \multirow[t]{2}{*}{ Decay ring circumference } & $\mathrm{m}$ & 6911.5 & 6911.5 & 6911.5 & 6911.5 & 26659 \\
\hline & & 24190 & 24190 & 24190 & 24190 & 26659 \\
\hline \multirow[t]{4}{*}{ Dipole magnetic field } & $\mathrm{T}$ & 5.984 & 3.580 & 5.317 & 3.191 & 8.327 \\
\hline & & 20.95 & 12.53 & 18.61 & 11.17 & 8.327 \\
\hline & & 5.984 & 3.580 & 5.317 & 3.191 & 8.327 \\
\hline & & 6.0 & 3.6 & 4.8 & 3.6 & $\cdots$ \\
\hline \multirow[t]{3}{*}{ Repetition time } & $\mathrm{s}$ & 15.6 & 9.6 & 14.4 & 9.6 & $\ldots$ \\
\hline & & 15.6 & 9.6 & 14.4 & 9.6 & $\ldots$ \\
\hline & & 9.346 & 7.178 & 48.18 & 16.7 & 32.29 \\
\hline \multirow[t]{3}{*}{ Total number of stored ions } & $10^{13}$ & 32.50 & 25.08 & 168.2 & 58.20 & 32.29 \\
\hline & & 32.50 & 25.08 & 168.2 & 58.20 & 32.29 \\
\hline & & 4.673 & 3.589 & 24.09 & 8.35 & 32.29 \\
\hline \multirow[t]{3}{*}{ Number of stored ions/bunch } & $10^{12}$ & 16.25 & 12.54 & 84.08 & 29.10 & 32.29 \\
\hline & & 16.25 & 12.54 & 84.08 & 29.10 & 32.29 \\
\hline & & 8.3937 & 19.282 & 57.668 & 19.984 & 362.16 \\
\hline \multirow[t]{3}{*}{ Stored beam energy } & MJ & 102.15 & 235.79 & 704.45 & 243.87 & 362.16 \\
\hline & & 102.15 & 235.79 & 704.45 & 243.87 & 362.16 \\
\hline & & 1.30 & 4.99 & 10.04 & 5.80 & 0.582 \\
\hline \multirow[t]{3}{*}{ Average intensity } & $\mathrm{A}$ & 4.52 & 17.428 & 35.06 & 20.22 & 0.582 \\
\hline & & 1.29 & 4.749 & 10.02 & 5.78 & 0.582 \\
\hline & & 227.9 & 875.0 & 1762 & 1017 & 0.582 \\
\hline \multirow[t]{3}{*}{ Peak intensity } & A & 792.3 & 3057 & 6150 & 3547 & 0.582 \\
\hline & & 792.3 & 3057 & 6150 & 3547 & 0.582 \\
\hline & & 9.872 & 11.41 & 66.18 & 25.20 & $\ldots$ \\
\hline \multirow[t]{3}{*}{ Power lost by decay } & $\mathrm{W} / \mathrm{m}$ & 34.78 & 40.00 & 232.3 & 88.54 & $\ldots$ \\
\hline & & 9.938 & 11.43 & 66.37 & 25.30 & $\ldots$ \\
\hline & & 38.6 & 4.64 & 23.0 & 8.27 & $\cdots$ \\
\hline \multirow[t]{2}{*}{ Limit collective effects/bunch } & $10^{12}$ & 139 & 16.7 & 82.5 & 29.7 & $\ldots$ \\
\hline & & 485 & 584 & 289 & 104 & $\ldots$ \\
\hline
\end{tabular}

to be stored in the decay ring to keep the production rates and the flux the same for the increased lifetimes of the isotopes at higher $\gamma$. The high intensities stored in the decay ring need additional studies concerning the safety and the beam control.

In the second scenario, the circumference is almost like the LHC circumference and the magnets are less than those of the LHC, however, the cost of a larger ring with a large number of elements is likely to be considerably higher. The stored beam energy is a concern.

The presence of collimators in the decay ring will increase the impedance seen by the beam, which will change the collective effect issues.
To conclude, the parameters for a decay ring at $\gamma=350$ are outstanding and need considerable studies. The stored beam energy increases by a factor of about 12. A rule of thumb to apply for acceptable heat deposition is that $10 \mathrm{~W} / \mathrm{m}$ is a tolerable value that can be handled without special research and studies. In Table XII is shown that this is exceeded for B and Li for all cases studied. We need 5 times more intensity in the machine to give higher neutrino rates due to the longer baseline. However for the $\gamma=350$ option with higher dipole field the heat deposition is exceedingly large. For the low-field dipole version of the $\gamma=350$ option the energy deposition is not higher than for the $\gamma=100$ option. 
The aim of the beta beam was to profit of existing infrastructures at CERN and integrate noninvasively with other beams and requirements. From the preliminary investigations that were possible within the EUROnu framework, it seems that the $\gamma=350$ beta beam would need too heavy investments to be feasible. In addition the cost/performance indicator for the facility would not compare favorably to very large and powerful facilities like the neutrino factory, which according to the EUROnu conclusion was considered the "facility of choice." The operation of the machine, running costs, safety etc. have not been addressed at this stage.

\section{Merging of the injected and stored beams}

One of the main issues of the beta-beam complex is the production of the ions. Moreover, the space-charge effects limit the maximum intensity we can accelerate in the PS [87]. That is why it is necessary to use an accumulation scheme in the decay ring to increase the stored intensities and then to reach the required neutrino fluxes. The injection compensates the losses which occur between two injection cycles.

In conventional schemes, a cooling scheme is used to damp the emittances between two successive injections, which enables to keep the emittance of the stored beam constant $[101,102]$. Unfortunately, it is not possible to use such a system for the decay ring. Electron cooling would require an electron beam of more than $50 \mathrm{MeV}$ [103] and electron capture would introduce a severe loss mechanism. Stochastic cooling [101] rates at the bunch intensities envisaged are orders of magnitude too slow and laserion cooling is neither possible because the ions are necessarily fully stripped and also there is no significant synchrotron radiation to provide damping.

\section{Dual rf system}

In the case of the beta-beam decay ring, the losses by $\beta$ decay are compensated by regular injections in the presence of the stored beam. The ions are injected at an energy slightly different from the one of the stored beam on their chromatic orbit. They are then merged with the stored beam by varying the voltage and the phase of two cavity families, of which one is at the frequency $40 \mathrm{MHz}$ and the other at the double frequency $80 \mathrm{MHz}$ [104]. Such a system has already been used to create halos or change the distribution of ions in the longitudinal phase space [105,106]. The principle was experimentally tested by injecting a hollow bunch [107].

The injection consists of three steps: (i) The stored beam is deflected to the blade of a septum magnet by using a system of four kickers. The fresh beam is injected "off momentum" and deflected by the septum magnet. The kickers are then switched off. (ii) Whereas the main rf cavity family, at the harmonic number $h=924(40 \mathrm{MHz})$, is on, the secondary family is still off. After a quarter turn in the longitudinal phase space, the injected beam is at the same energy as the stored beam but is late. (iii) The secondary cavity family, at the harmonic number $h=1848$ ( $80 \mathrm{MHz}$ ), is switched on. The rf program is then run to merge both beams.

The aim of the rf program is to merge both beams to get a unique beam at the reference energy. Four main steps which will be detailed in the following, can be identified [104,108]: (1) rotation of a quarter turn in the longitudinal phase space; (2) asymmetric merging with a constant area for the capture bucket; (3) symmetric merging; (4) progressive switching off of the second cavity family.

The whole rf program was calculated by using Mathematica [109]. We will respectively note $V_{1}, \phi_{1}$, $V_{2}$, and $\phi_{2}$ the voltage and phase of the first and second cavity. We will use $r=V_{2} / V_{1}$ the ratio between the voltages of the two cavities and $\phi_{21}=\phi_{2}-2 \phi_{1}$ the phase difference.

\section{Rotation of a quarter turn in the longitudinal phase space}

First, only the first cavity is on and its maximum voltage is $54 \mathrm{MV}$ for ${ }^{6} \mathrm{He}^{2+}$. The distribution of the incoming beam is assumed to be parabolic in the longitudinal phase space. Moreover, the beam is injected with a phase offset and tilted to optimize the capture at the injection. The fresh beam makes then a quarter turn (31 turns) in the longitudinal phase space. The capture bucket should be then centered on this phase to maximize the capture of the fresh beam.

\section{Asymmetric merging with a constant area for the left bucket}

The second cavity family is then switched on and the maximum voltage of the first cavity family is decreased from 54 to $35 \mathrm{MV}$ for ${ }^{6} \mathrm{He}^{2+}$ [110]. The voltages of both cavity families and their phases were calculated to maximize the number of ions trapped in the capture bucket. For synchronism reasons with the CERN-SPS, the azimuthal position of the center of the stored beam must stay the same from one injection to another.

The area of the capture bucket is taken to be equal to the full longitudinal emittance of the injected beam and will be kept constant through this process. If we choose a larger area for the capture bucket, the gain on the number of trapped ions at the injection is mitigated by the quicker blowup of the stored beam. We have chosen to reduce adiabatically the voltage of the second cavity, keeping the voltage of the first cavity constant. At the end of the asymmetric merging, the areas of both buckets are equal with $\phi_{21}=0^{\circ}$. The merging becomes symmetric.

\section{Symmetric merging}

During this step, the area of the left bucket is not kept constant whereas the buckets are kept symmetric $\left(\phi_{21}=0\right)$. 


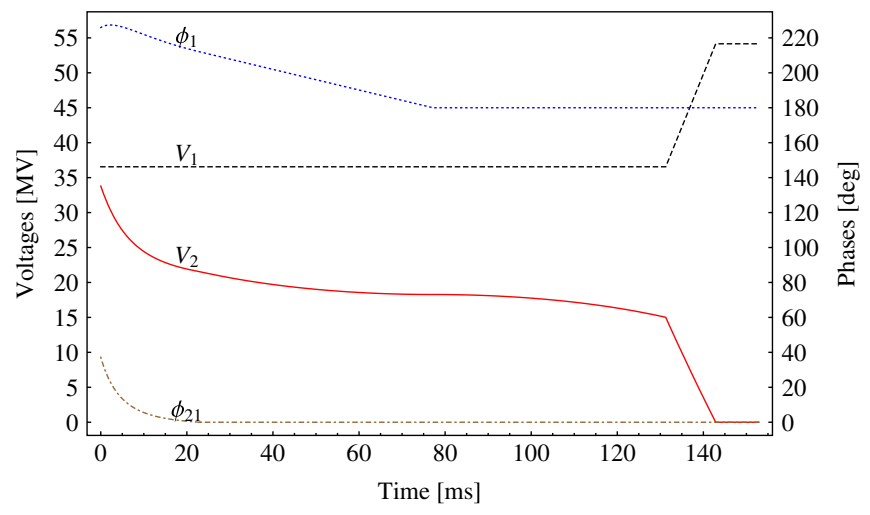

FIG. 42. Variations of the phases (in blue dotted for $\phi_{1}$ and in brown dot-dashed for $\phi_{21}$ ) and voltages (in red for $V_{1}$ and in black dashed for $V_{2}$ ) of the two cavity families during the rf program for helium.

At the end of the symmetric merging, $r=0.5$ and the synchrotron frequency is zero. We would need then an infinite time to perform an isoadiabatic merging. Therefore, it was chosen to linearly decrease $\phi_{1}$ up to $180^{\circ}$. The merging symmetric stops when $r=0.5$.

\section{Progressive switching off of the second cavity}

We linearly increase the voltage of the first cavity whereas we adiabatically switch off the voltage of the second cavity. The total time of the merging is $143 \mathrm{~ms}$. The variation of phases and voltages while the rf merging is given in Fig. 42 for ${ }^{6} \mathrm{He}^{2+}$.

\section{Simulation of the merging}

To illustrate the different steps of the merging, we have drawn the injected beam at different moments. The simulation was performed after applying the rf program illustrated in Fig. 42 [111]. First of all, the fresh beam is injected with an offset of 5\% in energy. This energy offset was determined to enable the insertion of a septum blade between the stored and injected beams at the injection [87]. In order to optimize the capture, the beam does not enter exactly in phase with the stored beam and was tilted. The longitudinal emittance of the entering helium 6 beam was taken equal to $1 \mathrm{eV} \mathrm{s}$ according to the FP6 database [112]. The beam is shown in Fig. 43 for several steps during the merging process. After one quarter turn, the beam is on momentum but is late compared to the stored beam. Since the beam is injected in a nonlinear region, the beam shape is strongly modified and has lost its initial elliptical shape. The second cavity is switched on and the asymmetric merging occurs. At the end of the asymmetric merging, most ions are still in the capture bucket. Some of them have generated a halo, which corresponds to the ions which were not initially in the capture bucket. The symmetric merging is then performed to go on the merging. At the end of the symmetric merging, the center of the fresh beam is at the origin. The area of the beam seems to have doubled. In fact, during the symmetric merging, the capture bucket is merged with its symmetric centered on the origin. At the end of the merging, both buckets have merged and according to Liouville's theorem, the area has doubled. The second cavity is then progressively switched off to obtain the final beam. The beam is then stored until the rf merging is performed again for the next injection.

\section{Barrier buckets}

An alternative injection scheme was studied in [113]. The idea is to use voltage barriers to squeeze all incoming ions from SPS into one so-called barrier bucket. A high intensity of ions inside the bucket increases the neutrino flux and thereby also the sensitivities of the experiment. The time spread of the bucket would however decrease the sensitivities since that would worsen the suppression factor of the experiment. The question was then to study whether it is possible to optimize between the ion intensity kept inside the bucket and the duty cycle that the bucket occupies so that the sensitivities comply with the requirements for the beta beam.

The conclusion was that for a bucket with a size corresponding to $4 \%$ (2\%) duty cycle of the DR, barriers with filling times $1 / 2$ (or $300 \mathrm{~ns}$ ) (1/4 or $150 \mathrm{~ns}$ ) of the SPS cavities are necessary so that not more than $80 \%$ of the ions escape the bucket before they decay. By additionally assuming an ion production rate of $10^{14}$ ions/s for both ${ }^{8} \mathrm{~B}$ and ${ }^{8} \mathrm{Li}$ and no charge intensity limit in the SPS a much too optimistic (anti)neutrino flux of $\left(7.57 \times 10^{18}\right) 3.25 \times$ $10^{18}$ was estimated. Even with these fluxes sensitivity plots of $\delta_{\mathrm{CP}}$ and $\theta_{13}$ show that a suppression factor for the atmospheric background less than $1 \%$ would be needed. Since that suggests unrealistic rf cavities in the DR the conclusion is that the barrier bucket method is not optimal for the FP7 framework. The barrier bucket scheme would be interesting again only if the SF could be significantly enlarged.

\section{Decay ring rf system design}

In order to merge the stored bunches with the injected bunches in the decay ring $40 \mathrm{MHz}$ rf is required with varying rf phase, as well as $80 \mathrm{MHz}$ rf [110]. The required voltage is up to $55 \mathrm{MV}$ for the $40 \mathrm{MHz}$ system and $35 \mathrm{MV}$ for the $80 \mathrm{MHz}$ system. The stored current is $60 \mathrm{~A}$ for a helium beam (for the gamma $=100$ design) hence the beam induced voltage is larger than can be controlled effectively with a realistic rf power. In order to reduce the beam loading it is necessary to modify the rf system to detune the cavity and use a lower $R / Q$ cavity to minimize the detuning. This approach has been studied for use in the beta beam decay ring. 

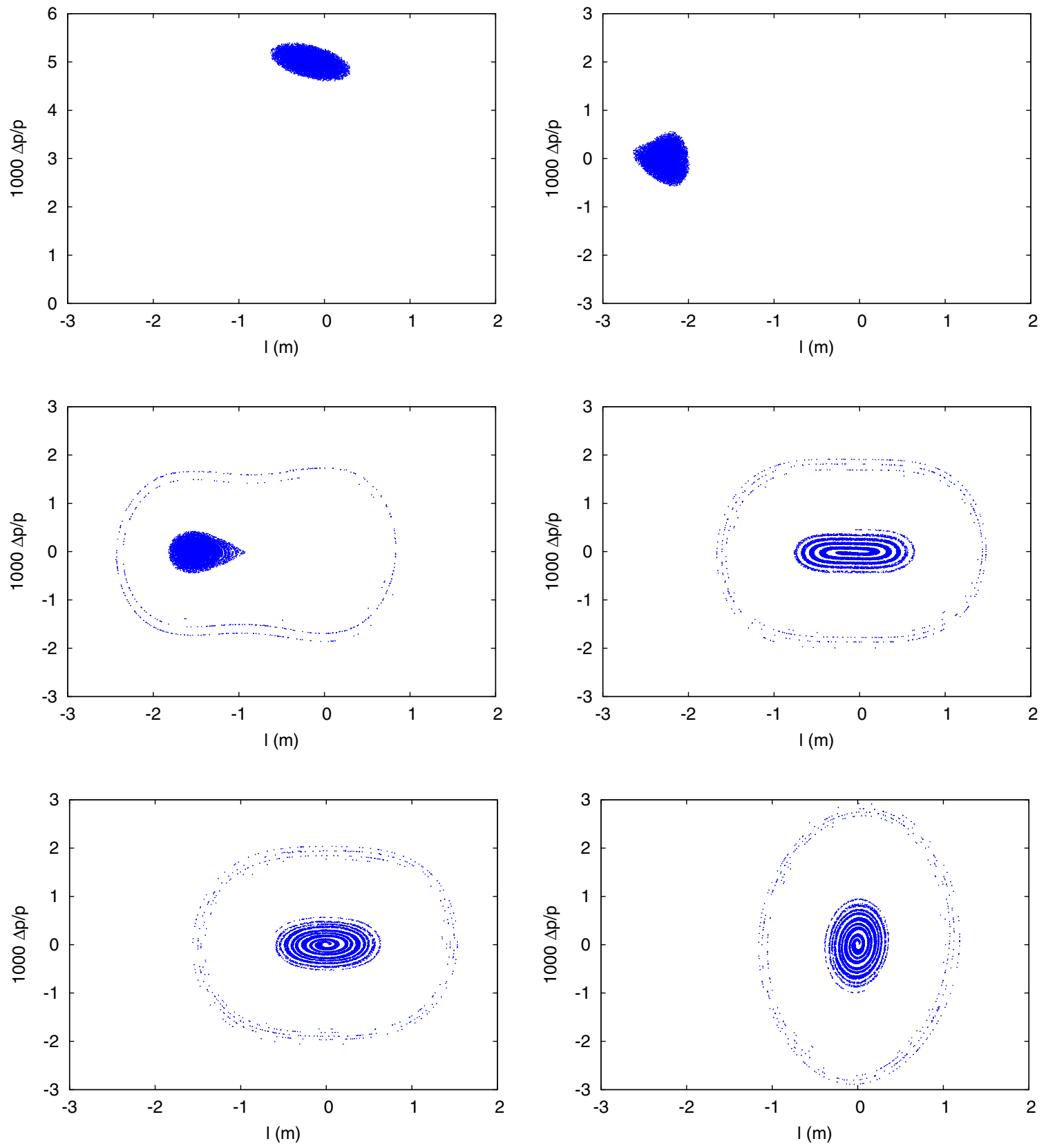

FIG. 43. Fresh helium beam in the longitudinal phase space after entering into the decay ring, after one quarter turn, while the asymmetric merging, while the symmetric merging, at the end of the symmetric merging, at the end of the merging.

\section{Detuning}

As mentioned previously the beam phase varies between 40 degrees and 0 degrees from quadrature with the rf. When the beam is in close to quadrature the beam loading is strongly capacitive, adding additional inductance to the cavity, by detuning it, can compensate for this [87]. In principle the phase shift due to the rf being run at the wrong frequency is equal and opposite to the phase shift caused by the beam. For a cavity of angular frequency, $\omega$, geometric shunt impedance, $R / Q$, gap voltage, $V_{g}$, and a beam of current, $I_{b}$, the phase shift is

$$
\Delta \phi=\arctan \left(\frac{I_{b} R}{V_{g} Q}\right)
$$

Hence the detuning in frequency should be

$$
\Delta \omega \approx \omega_{0}\left(\frac{I_{b} R}{2 V_{g} Q}\right) .
$$

The rf then only needs to compensate for the beam power deposited in the cavity and not change the cavity phase, and the generator can be matched to the cavity. 
The power deposited in the cavity by the beam arriving at phase $\phi_{s}$ is given by

$$
P_{b} \approx I_{b} V_{g} \sin \phi_{s} .
$$

Hence, for quadrature no power is seen to be induced by the beam. This is because the phase of the cavity is slightly adjusted so that the power actually induced by the cavity is canceled by the acceleration of the cavity. The beam does not experience this phase shift as the induced power would alter the phase of the cavity anyway.

For the beta beam decay ring $\left(I_{b}=60 \mathrm{~A}\right)$ using a cavity similar to the PS $40 \mathrm{MHz}$ cavity [114] (R/Q $=33 \Omega$, $Q_{0}=18000$ ), and using a $408 \mathrm{kV}$ gap voltage the required detuning is around $0.1 \mathrm{MHz}$. This results in a required $\mathrm{rf}$ power of $761 \mathrm{~kW}$ to keep the cavity on voltage compared to 269 MW required without detuning for a matched cavity as the cavity is far off resonance, although this could be reduced to around $6 \mathrm{MW}$ by lowering the $Q$ and hence increasing the bandwidth.

However, immediately after the injection of a new top up bunch the rf phase is 40 degrees from quadrature which means the power deposited in the cavity by the beam is significantly higher than the power lost in the cavity walls. Also as the beam is very long not all of the bunch will be in the linear part of the sine wave. The highest power tetrode available is $10 \mathrm{MW}$, it is assumed that it is possible to double this power by using a diacrode hence we assume a maximum power of $20 \mathrm{MW}$. For a neon beam the current is 4 times higher but the voltage is almost half, hence twice the $\mathrm{rf}$ power is required, hence to use the same system for He we need to limit the rf power to $10 \mathrm{MW}$. For a $60 \mathrm{~A}$ beam the maximum cavity voltage is $260 \mathrm{kV}$ at this phase and the beam induced power per cavity will be $10 \mathrm{MW}$. The cavity can be matched by setting the external $Q, Q_{e}$ [115]

$$
\beta=\frac{Q_{0}}{Q_{e}}=1+\frac{P_{b}}{P_{c}},
$$

where $Q_{0}$ is the Ohmic $Q$ of the cavity and $P_{c}$ is the cavity wall losses. This gives an external $Q_{e}$ of 102 to match the cavity. This results in a required power of

$$
P_{q}=\frac{\left[(1+\beta) P_{c}+P_{b}\right]^{2}}{4 \beta P_{c}} .
$$

However, when the beam is not there $\left(P_{b}=0\right)$ the generator mismatch is large and $6.3 \mathrm{MW}$ is still required to keep the cavity at constant voltage which is a large average power. To reduce this we can make use of the fact that we are planning to use two rf sources at different frequencies to use a higher external $Q$ coupler for the generator filling at the detuned frequency, if we can make the $40 \mathrm{MHz}$ generator's coupler narrow band or with a filter so it has a high external $Q$ at the detuned frequency. Such a scheme would require further investigation and in this paper we assume that it is possible. The advantage of a low external $Q$ is that when the stored current varies the change in detuned frequency is smaller than the cavity bandwidth hence tuning can be slow without further increases to the required power. The second coupler would be matched to the Ohmic $Q$ of the cavity and hence the required power to fill the cavity would be $140 \mathrm{~kW}$. To achieve $35 \mathrm{MV}$ (which is the required voltage when the phase is 40 degrees from quadrature) we would need 135 cavities at $260 \mathrm{kV}$, requiring a total average rf power of 40-50 MW depending on the time between injections. The cavities must also be able to increase their voltage to $408 \mathrm{kV}$ during normal operation to reach $55 \mathrm{MV}$ when the phase has returned to quadrature as mentioned previously.

\section{Low $R / Q$ cavity design for neon beams}

We will always wish to feed at $40 \mathrm{MHz}$ when the beam is present however the detuning varies as the current, phase and voltage of the cavity vary. This is necessary as the detuning also varies as the voltage is ramped from 35 to $55 \mathrm{MV}$, the phase varies from 40 to 0 degrees and the current is stacked up over time. This means that the detuned frequency will vary from $40 \mathrm{MHz}$ down to $39.9 \mathrm{MHz}$ for a 60 A current and a voltage of $408 \mathrm{kV}$. In order to keep the filling frequency at a high $Q$, we would want to keep the cavities natural frequency constant, this means when the beam is present we will have to fill off resonance. This is acceptable in practice as the cavity bandwidth is so large the additional power required for this is small. The cavity will be filled at the lower frequency and then driven at $40 \mathrm{MHz}$ when the beam arrives. This can result in transient phase shifts due to the two frequencies existing in the cavity. Such a system requires in depth circuit models to fully understand but for now we use a simple approximation as a worst case scenario.

The cavity frequency varies by $0.1 \mathrm{MHz}$, and over a train of 20 bunches the frequency difference between a $40 \mathrm{MHz}$ signal and a $39.9 \mathrm{MHz}$ signal is $18 \mathrm{deg}$. The actual phase shift in the cavity will be lower than this as the signal is mixed but this is likely to still be an issue and will require additional rf power to correct. However for a neon beam this detuning increases by a factor of 8 which is a bigger concern. A reduced $R / Q$ will reduce the detuning required and hence the phase shift proportionally. As we would want to use the same linac for both beams, the neon beam would also require this low $R / Q$ cavity.

If we reduce the $R / Q$ down to $2 \Omega$ the maximum phase shift is reduced by a factor of 16.5 which is more manageable. This has the added advantage that it will also likely reduce the $R / Q$ of any higher order modes depending on the exact cavity geometry which may also be a limiting factor. This also give a more realistic external Q of 1685 , as opposed to 102 which is required for an $R / Q$ of $33 \Omega$. This does not affect the required power with beam as the $Q_{e}$, detuning and $R / Q$ all vary proportionally. 


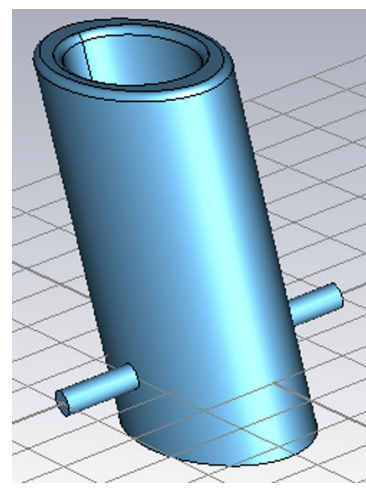

FIG. 44. The cavity geometry.

However, if we use a higher $Q$ coupler to fill the cavity without beam than with beam then the lower $R / Q$ means 2.3 MW would be required to fill the cavity if normal conducting. It is hence required that the cavity is superconducting. In this case $1 \mathrm{~kW}$ is required to fill the cavity if an external $Q$ of $10^{7}$ is used.

Initially it was assumed that a low $R / Q$ could be achieved by using a cavity similar to the PS $40 \mathrm{MHz}$ cavity and shortening the gap. Indeed as the gap gets shorter the $R / Q$ does drop, however very small gaps are required for an $R / Q$ of $2 \Omega$, and the capacitance becomes very large lowering the cavity frequency. A $1 \mathrm{~mm}$ gap would only reduce the $R / Q$ to 4.5 . This results in the ratio of the peak electric field to the accelerating voltage increases sharply reducing the maximum operating voltage below a usable level. The gap required is also too small to be reliably manufactured and the cavity frequency becomes very sensitive to the gap width.

A low $R / Q$ cavity can be realized by using a conventional quarter-wave resonator and moving the point where the beam traverses the cavity to a location of lower voltage. This can be achieved by moving the beam towards either end of the cavity. This will unfortunately also provide a transverse electric or magnetic field that will provide a transverse kick to the bunch as the accelerating and deflecting fields are 90 degrees out of phase with each other. This however can be canceled by flipping the cavity orientation every other cavity.

The cavity was designed and optimized using CST's eigenmode solver [116]. We optimize the cavity to reduce peak surface fields, the cavity size, and the transverse kick. The final cavity design, shown in Figs. 44 and 45, has been simulated using CST MICROWAVE STUDIO. It is $1 \mathrm{~m}$ long and has a height of $2 \mathrm{~m}$. However, as the cavities must flip orientation every other cavity the total width will be twice this at $4 \mathrm{~m}$, making the total cryostat width around $4.5-5 \mathrm{~m}$ wide. The peak electric field at the design voltage for the $\mathrm{He}$ beam of $408 \mathrm{kV}$ is $12 \mathrm{MV} / \mathrm{m}$. The $R / Q$ is $2 \Omega$, and the peak magnetic field is $32 \mathrm{mT}$.

The ratio of transverse to longitudinal voltage is 4.5 which is rather large resulting in a $1.8 \mathrm{MV}$ transverse voltage per cavity, hence the cavities do require to alternate position to give a net cancellation of the transverse kick. It should be noted the cavities are operated close to quadrature and hence the full transverse voltage is not seen by the beam and the cavity acts more like a crab cavity [117]. An alternate design which does not have this transverse kick can be realized by overlapping two of these quarterwave cavities to make something akin to a half-wave resonator, as shown in Fig. 46. This cavity is similar to the first accelerating mode in a double-quarter-wave resonator cavity [117].

The transverse component of the two quarter-wave rods cancel out while the longitudinal components sum together, as shown in Fig. 46. The $R / Q$ can be lowered by increasing the gap between the two quarter-wave structures and the peak fields remain roughly the same as the quarter-wave cavity. Unfortunately this requires a longer structure and the height from the beam axis increases to $2.1 \mathrm{~m}$, making the total height $4.2 \mathrm{~m}$ which would be rather difficult to manufacture.

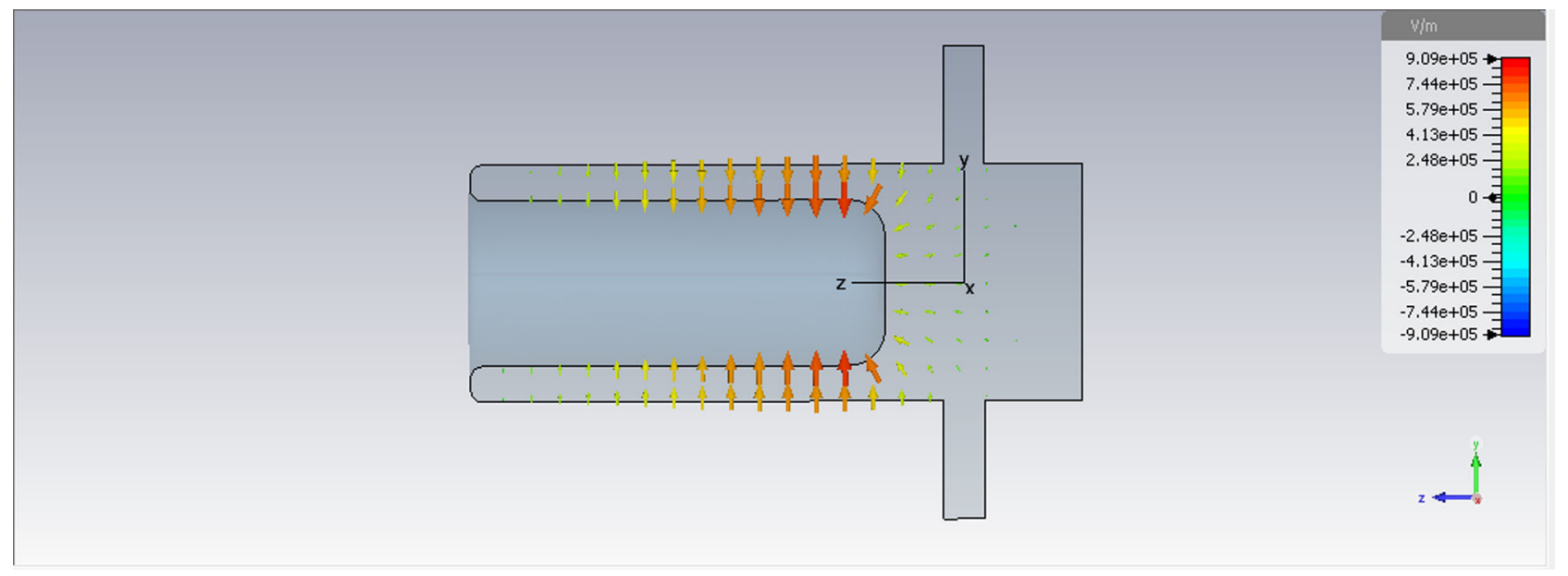

FIG. 45. The cavity electric field profile for the decay ring $40 \mathrm{MHz}$ rf system. 


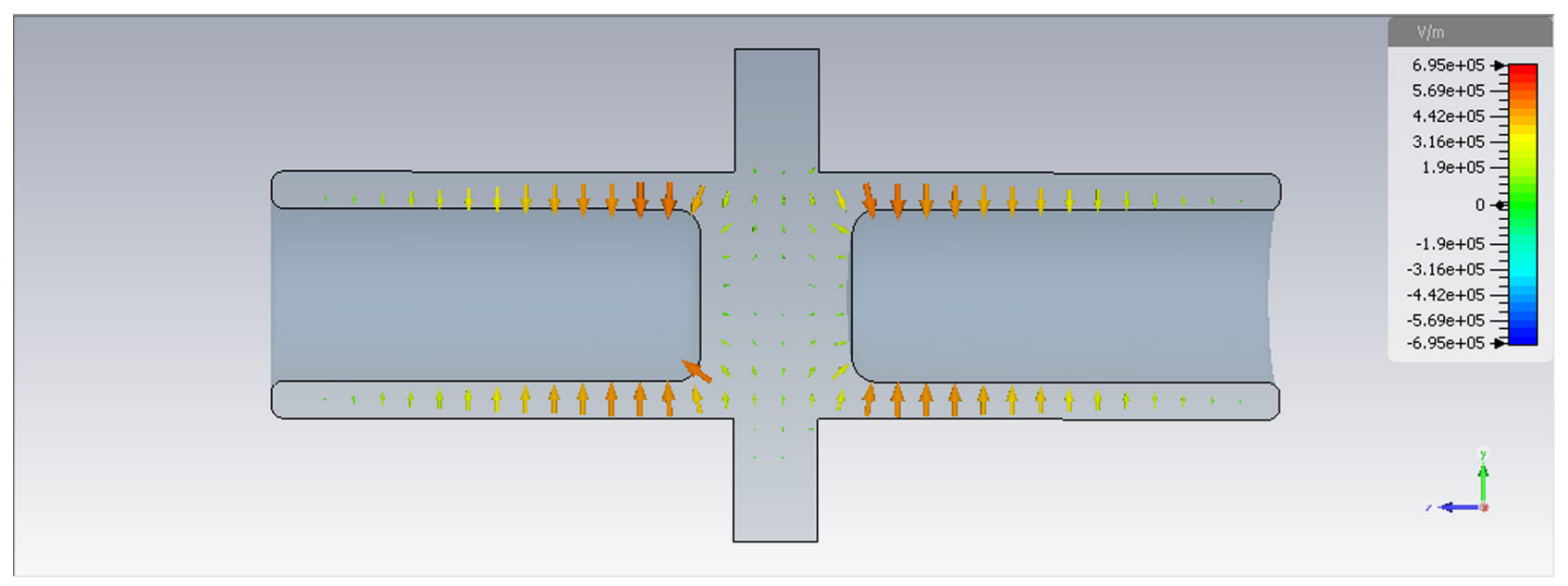

FIG. 46. A symmetric cavity with no transverse kick with its electric fields.

A quarter-wave cavity with an $R / Q$ of $2 \Omega$ is proposed. This allows an operating voltage of $408 \mathrm{kV}$, hence 250 cavities are required. The external $Q$ of the cavity should be $10^{7}$, hence the power required to fill the cavity to $408 \mathrm{kV}$ is $1 \mathrm{~kW}$. If the beam current is $60 \mathrm{~A}$ then the required detuning should only be $5.9 \mathrm{kHz}$, hence the phase shift due to this is less than 1 degree which is acceptable. When running on resonance the required power to keep phase and amplitude on specification with the beam in quadrature is $6.2 \mathrm{MW}$. However, this power is only required during the pulse which is $500 \mathrm{~ns}$ long, the other $98 \%$ of the time, between the trains, only $600 \mathrm{~kW}$ is required hence the total average power per cavity is only $156 \mathrm{~kW}$ which is more reasonable. This takes the total average power for 135 cavities to around $21 \mathrm{MW}$.

\section{Recirculating beam}

As the beam is recirculating the rf needs to be at the correct phase when the train returns. The revolution frequency of the decay ring is $43 \mathrm{kHz}$ which is much less than the maximum detuning. This makes it impossible to make the detuned frequency a harmonic of the beam frequency, hence a phase advance must be added to the rf phase at the end of the train in order for the phase to return to the correct value when the train returns to the cavity.

The rf system will have to run at two different frequencies depending on if the beam train is passing through the cavities or not and this will complicate the low level RF (LLRF) system. It is proposed that the LLRF system would operate using a $40 \mathrm{MHz}$ reference and digitally add a phase advance correction to the measured phase to correct for the detuned frequency of operation.

\section{Results}

A solution is proposed for the decay ring $40 \mathrm{MHz}$ rf system. The proposed system requires 135 cavities, and a total $\mathrm{rf}$ peak power of $1350 \mathrm{MW}$ and a total average $\mathrm{rf}$ power of $21 \mathrm{MW}$ for a He beam. For a neon beam the number of cavities remains constant but the peak and average power are doubled as the current goes up by a factor of 4 and the voltage is halved.

The total cavity height is $2.0 \mathrm{~m} / 2.1 \mathrm{~m}$ and has a length of $1 \mathrm{~m}$ hence a cryostat is likely to be $4.5-5 \mathrm{~m}$ wide and $1.5 \mathrm{~m}$ long. For 135 cavities the total $40 \mathrm{MHz}$ rf section length will be about $200 \mathrm{~m}$ long, the $80 \mathrm{MHz}$ system has not been explicitly included but is likely to be similar, hence the total rf section will be around $800 \mathrm{~m}$ long and the power required will double.

\section{E. Beam losses}

For this study, only two beam losses were looked at: (i) losses due to the $\beta$ decay of the radioactive ions; (ii) losses due to the rf merging after the injection. Some injected ions are not captured and are then lost while the merging. Moreover, the merging process blows up the longitudinal emittance which means some particles will be collimated at the end of the injection.

The decay losses occur continuously and anywhere in the ring whereas the losses due to the rf merging occur mostly after the injection and where the momentum acceptance is the lowest.

\section{Decay losses}

The aim of the decay ring is to store high intensity and high energy beams of $\beta$ radioactive ions until their decay. A first study was realized in order to quantify the average activation of the concrete walls and their impact on the public health [118]. First estimations show that these values are under the allowed ones. The superconducting magnets are sensitive to the beam losses, and the energy deposition from the decay products in the magnet coils has to be limited. Protection of the superconducting coils is important and may require that the decay ring lattice be adapted 
to minimize the power deposition. When an ion decays, its momentum variation is negligible (the energy taken by the electron and the antineutrino is low compared to the energy of the secondary ion) whereas its charge number increases or decreases by 1 . Therefore, its magnetic rigidity changes. In this part, we shall respectively use the subscripts 0 and 1 to refer to the primary and secondary ions. We shall consider as a decay product only the secondary ion which comes from the decay of the radioactive ions. The relative difference of magnetic rigidity between the secondary ion and the primary ion is then

$$
\delta=\frac{\Delta(B \rho)}{B \rho}=\frac{Z_{0}}{Z_{1}}-1 .
$$

The ions ${ }^{6} \mathrm{He}^{2+},{ }^{18} \mathrm{Ne}^{10+},{ }^{8} \mathrm{Li}^{3+}$ and ${ }^{8} \mathrm{~B}^{5+}$ respectively decay into ${ }^{6} \mathrm{Li}^{3+},{ }^{18} \mathrm{~F}^{9+},{ }^{8} \mathrm{Be}^{4+}$ and ${ }^{8} \mathrm{Be}^{4+}$ and the magnetic rigidity variation is respectively $\delta=-1 / 3, \delta=+1 / 9$, $\delta=-1 / 4$, and $\delta=+1 / 4$. The variation is so large that the decay products are quickly lost after deflecting in the dipoles, which makes the extraction impossible in the arcs.

Since the revolution time ( $23 \mu \mathrm{s})$ is low compared to the half-life time of the stored ions in the laboratory frame $(\approx 100 \mathrm{~s})$, we can assume that the number of ions lost per meter is the same anywhere in the structure. The probability $P(t)$ that an ion did not decay after $t$ seconds is then

$$
P(t)=2^{-\frac{t}{\gamma \tau}},
$$

where $\tau$ is the half-time of the ion at rest.

With an initial number of ions of $N_{0}$, the number of ions which decay per second is then $-N_{0} P^{\prime}(t)$. The maximum power lost by decay per meter $\mathcal{P}_{m}$ is then

$$
\mathcal{P}_{m}=\frac{\gamma-1}{\beta \gamma} \frac{N_{0} E_{0} \ln (2)}{2 \pi R \tau}
$$

By using the stored intensities given in Table VIII, we obtain the power lost by decay per meter in the structure are respectively $9.872,11.41,66.18$, and $25.20 \mathrm{~W} / \mathrm{m}$ for helium, neon, lithium, and boron.

The decay losses are essentially deposited after bending magnets. We therefore assume, for our first estimations, that decay in straight sections, including the rf system, can be handled using a special dump after a first dipole at the end of the two long straight sections and absorbed correctly. The first approach to handling the decay losses in the arcs was to insert absorbers after the dipoles. The lack of flexibility and the significant impedance increase, suggested that other solutions should be studied.

Since the decayed ions are deflected essentially only in the magnet midplanes, it was decided to use dipoles and quadrupoles that are coil-free on the midplanes. In this way, the decay products will impinge the magnet in a region without any superconducting coil. The material used in the midplanes would be a low- $\mathrm{Z}$ material to avoid heat deposition near the coil. A design should be studied where the heat can be deposited in, for example, an absorber in the magnet collars and transported away by an efficient cooling system.

Since the rms vertical beam size is about $2.5 \mathrm{~mm}$ and the magnet aperture is $60 \mathrm{~mm}$, the opening angle can be $5^{\circ}$. First studies of such a dipole for the beta beam decay ring were shown in $[119,120]$. First designs of quadrupoles with coil-free midplanes were shown on [92].

\section{Injection losses}

After each merging, the beam blows up in the longitudinal phase space due to Liouville's theorem. Since there is no cooling quick enough to compensate the growth of the longitudinal emittance, it is necessary to collimate in energy between two successive injections. Since some particles with a relative momentum difference of $2.5 \%$ hit the septum blade at the injection, it was decided to collimate at $\delta_{C}=2.5 \%$. Because of the large average power to collimate, it was decided to use a multistaged collimation section.

Since the particles have a betatron amplitude, some of them will hit the collimator although their energy difference is lower than the energy at which we want to collimate. We have evaluated the efficiency of the collimation by tracking a large number $N$ of particles in the longitudinal phase space for 40 merging processes. At the end of the merging, the voltages and phases of the cavities do not vary. A particle $P_{i}$ of the beam is considered. The coordinates of the particle in the longitudinal phase space after $n$ injections is $[l(n), \delta(n)]$. The maximum relative energy difference $\delta_{i}(n)$ that $P_{i}$ can reach during the synchrotron motion before the next merging is calculated. If $\delta_{i}(n)>\delta_{C}$ then $P_{i}$ is lost. If $\delta_{i}(n)<\delta_{C}$ then $P_{i}$ hits the collimator if its normalized betatron amplitude is greater than $k_{i}(n)=$ $\left[\delta_{C}-\delta_{i}(n)\right] \frac{D_{n, x, C}}{\sqrt{\epsilon_{x}}}$ where $D_{n, C, x}=\frac{D_{x}}{\sqrt{\beta_{x}}}$ at the collimation point. Since we know the transverse distribution, we know the probability $p\left[k_{i}(n)\right]$ that $P_{i}$ has a normalized betatron amplitude smaller than $k_{i}(n)$. The number of ions $N_{\text {ion }}\left(D_{n, x, C}, n\right)$ which survived after $n$ injection cycles can be then evaluated by

$$
N_{\text {ion }}(n)=\frac{1}{N} \sum_{i=1}^{N} N_{\text {inj }} p\left[k_{i}(n)\right] 2^{\frac{-n T}{\gamma \tau}} .
$$

The total number of ions lost by decay $N_{d}\left(D_{n, x, C}\right)$ and the one lost by momentum collimation $N_{C}\left(D_{n, x, C}\right)$ between two injections is then

$$
N_{d}\left(D_{n, x, C}\right)=\left(2^{\frac{T}{\gamma \tau}}-1\right) \sum_{n=0}^{\infty} N_{\text {ion }}(n+1),
$$




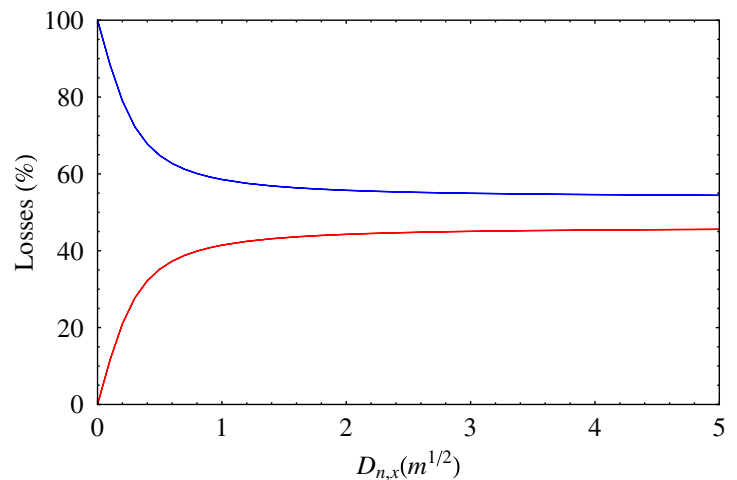

(a) Helium

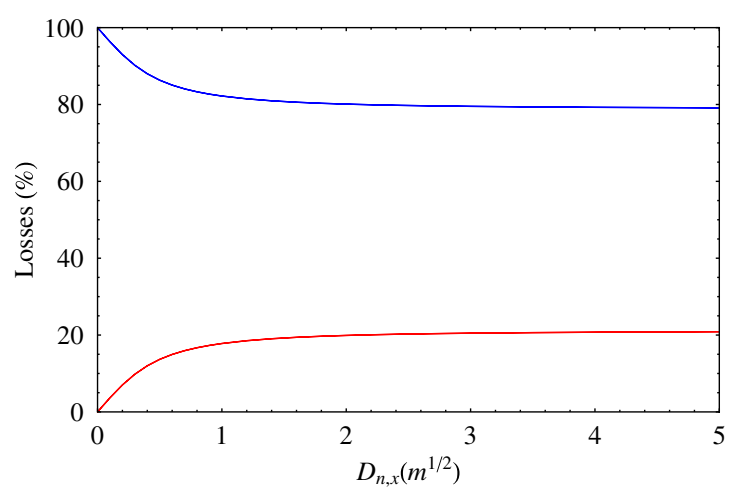

(b) Neon

FIG. 47. Sum of the losses by collimation (red) and by decay (blue) versus the normalized dispersion at the primary collimator for a Gaussian beam.

$$
N_{C}\left(D_{n, x, C}\right)=\sum_{n=0}^{\infty}\left[N_{\text {ion }}(n)-N_{\text {ion }}(n+1) 2^{\frac{T}{\gamma \tau}}\right] .
$$

That enables one to plot the repartition of the losses between two injections versus $D_{n, x, C}$ (Fig. 47). The losses approach an asymptote for $D_{n, x, C}$ equal to a few $\mathrm{m}^{1 / 2}$.

\section{F. Collective effect studies}

High intensity ion beams are foreseen for the beta beam project. High intensity bunches can have a non-negligible amount of charges which could cause the particles to interact with each other and with the vacuum chamber, so-called "collective effects." Collective effects could limit the final performance of the accelerators. The studies of instabilities of all machines in the beta beam complex is therefore a crucial part of the project.

\section{Direct space-charge effect}

Although the beam is relativistic, the direct space charge is not negligible due to a charge of several microcoulombs per bunch. For a beam with a Gaussian transverse distribution and a parabolic longitudinal distribution, the incoherent tune shift is [121]

$$
\Delta Q_{x, y}=-\frac{3}{4} \frac{Z^{2}}{A} \frac{N_{\text {bunch }} r_{0} R}{L_{\text {beam }} \beta^{2} \gamma^{3} \epsilon_{x, y}\left(1+\sqrt{\frac{\epsilon_{y, x} Q_{x, y}}{\epsilon_{x, y} Q_{y, x}}}\right)},
$$

where $\epsilon_{x, y}$ is the transverse rms emittance and $r_{0}$ the classical proton radius.

The tune shifts for the different ion species are summed up in XIII. Since Neon ions have the largest charge number, their tune shift is the greatest. The tune shift is then at the limit we can manage for a storage ring. A special care should be taken to verify if the beam is perturbed by its own potential. This study has not been pursued yet. A solution to mitigate the direct space-charge effects could be to inject a beam with a larger transverse emittance or a mismatched beam.

\section{Transverse broadband resonance impedance}

The electric field lines from the bunch cause an image charge to travel along with the bunch in the vacuum chamber [122]. Geometrical or material variations of the vacuum chamber cause the image charge to radiate electromagnetic fields called wakefields. The beam then loses energy that heats the chamber and feeds the wakefields that could be trapped and remain oscillating for some time. If the wakefields last for the duration of the bunch $(\approx 100 \mathrm{ps})$ particles in the "tail" of the bunch can interact with the wakefields due to the particles in the "head" of the bunch and cause single bunch instabilities. Wakefields that last until the next bunch $(\approx n s)$ could cause multibunch instabilities. For the studies shown in this paper we have focused on single bunch instabilities only.

The action of the wakefields is described by the wake potential, $W(t)$, in the time domain and by the impedance, $Z(\omega)=\mathcal{F}[W(t)]$, in the frequency domain. If the wakefields are caused by resistivity of the vacuum chamber material the impedance is called resistive wall impedance, $Z^{r w}(\omega)$, but is beyond the scope of this study. Here we will report on studies of impedances caused by wakefields trapped in cavities of the vacuum chamber, so-called resonance impedances, $Z^{\text {res }}(\omega)$. If the quality factor is $Q=$ $R \sqrt{C / L}$ and the resonance frequency is $\omega_{r}=1 / \sqrt{L C}$ the resonance impedance can be modeled as an RLC circuit [123] in the transverse plane as

$$
Z_{\perp}(\omega)=\frac{R_{\perp} \frac{\omega_{r}}{\omega}}{1+i Q\left(\frac{\omega_{r}}{\omega}-\frac{\omega}{\omega_{r}}\right)},
$$

where $R_{\perp}$ is the transverse shunt impedance. $R_{\perp}$ is a value indicating the total divergence from a perfectly smooth vacuum pipe around the whole ring. The value for SPS is about $20 \mathrm{M} \Omega / \mathrm{m}$ but for the DR this is an unknown value since the DR is yet a nonexisting machine. Since the DR is a modern machine we can assume it will have a smooth vacuum pipe design and since it will not be as general machine as SPS it will also have less number of kickers. We 
TABLE XIII. Laslett tune shifts for the different ion species in the decay ring.

\begin{tabular}{lcrrrr}
\hline \hline & Units & He & Ne & Li & B \\
\hline Charge number Z & & 2 & 10 & 3 & 5 \\
Mass number A & & 6 & 18 & 8 & 8 \\
Rest energy & $\mathrm{GeV}$ & 5.606 & 16.77 & 7.471 & 7.472 \\
$\gamma$ & & 100.0 & 100.0 & 100.0 & 100.0 \\
Horizontal tune & $\mathrm{m}$ & 18.23 & 18.23 & 18.23 & 18.23 \\
Vertical tune & $\mathrm{m}$ & 18.16 & 18.16 & 18.16 & 18.16 \\
Number of ions/bunch & $10^{12}$ & 4.673 & 3.589 & 24.09 & 8.35 \\
Number of stored & $10^{12}$ & 4.457 & 3.423 & 3.162 & 2.895 \\
$\quad$ ions/bunch & & & & 0.110 & 0.110 \\
Horizontal rms emittance & $\mu \mathrm{m} \mathrm{rad}$ & 0.110 & 0.110 & 0.079 & 0.079 \\
Vertical rms emittance & $\mu \mathrm{m} \mathrm{rad}$ & 0.079 & 0.079 & 1.933 & 1.933 \\
Total bunch length & $\mathrm{m}$ & 1.933 & 1.933 & -0.204 & -0.213 \\
Laslett tune shift & & -0.025 & -0.164 & & \\
\hline \hline
\end{tabular}

can therefore assume a factor 20 better transversal shunt impedance; $R_{\perp}^{\mathrm{DR}} \approx 1 \mathrm{M} \Omega / \mathrm{m}$ [124]. So far we have only studied short-lived resonance wakefields, i.e., broadband $(Q=1)$ impedances in the transverse plane. There are many different types of collective effects that could lead to beam instability and limit the maximum number allowed ions per bunch, $N_{b}^{\text {th }}$, but this study is constrained to transverse broadband resonance impedances.

\section{Methods to estimate bunch intensity limits}

Three different methods have been used to estimate $N_{b}^{\text {th }}$, the maximum number ions that could populate a bunch without too big a chance of severe beam instability.

One approach is to use the peak current values of the bunch current and momentum spread as input to a coasting beam formula. This gives an expression for the intensity limit that we will call the coasting beam equation [100]:

$$
N_{b_{x, y}}^{\mathrm{th}}=\frac{32}{3 \sqrt{2} \pi} \frac{Q_{x, y}|\eta| \varepsilon_{l}^{2 \sigma} \omega_{r}\left(1+\omega_{\xi_{x, y}} / \omega_{r}\right)}{c Z^{2} \beta^{2} R_{\perp}} .
$$

Here $c$ is the speed of light in vacuum, $\omega_{\xi_{x, y}}=$ $\xi_{x, y} Q_{x, y} \omega_{\text {rev }} / \eta$ and all other parameters are given in Table XIV.

MOSES [125] solves an integral equation in the frequency domain to give the rise time, $\tau$, of the instabilities for different head-tail modes as a function of the bunch intensity. The limit, $I_{b}^{\text {th }}$, is given by the most crucial head-tail mode after defining the maximum allowed growth rate, $(1 / \tau)^{\text {th }}$. To reach the ion equivalent intensity threshold we divide by a factor $Z ; \bar{I}_{b}^{\text {th }}=I_{b}^{\text {th }} / Z$. The maximum allowed number of ions per bunch is then given by the conversion $N_{b}^{\text {th }}=T_{\text {rev }} \bar{I}_{b}^{\text {th }} / Z e$. The green curve in Fig. 48 shows growth rates, $1 / \tau$, as a function of bunch populations, $N_{b}$, from MOSES. The maximum allowed number ions per bunch, $N_{b}^{\mathrm{th}}$, according to MOSES, is indicated by the green vertical line, for this example when $(1 / \tau)^{\text {th }}$ was chosen to $20 \mathrm{~Hz}$ (indicated by red dotted line).
The third method uses the multiparticle tracking code HEADTAIL [126] where a bunch of macroparticles is sliced longitudinally and the impedance is assumed to be localized at a few positions around the ring. At each impedance location, each slice leaves a wakefield behind and gets a kick by the field generated by the preceding slices. The bunch is then transferred to the next impedance location via

TABLE XIV. Input parameters above the first line. Assumed transversal impedance parameters between the lines. Calculated parameters below the last line. These parameters are the same for the different isotopes.

\begin{tabular}{lcc}
\hline \hline Parameters & Description & DR \\
\hline$N_{B}$ & Number bunches & 20 \\
$h$ & Harmonic number & 924 \\
$C[\mathrm{~m}]$ & Circumference & 6911.6 \\
$\ell_{\text {eff }}$ & Effective straight section & $37.2 \%$ \\
$\rho[\mathrm{m}]$ & Magnetic radius & 155.6 \\
$\gamma_{\mathrm{tr}}$ & Gamma transition & 16.772 \\
$\gamma$ & Relativistic gamma & 100.0 \\
$Q_{x}$ & Horizontal tune & 18.23 \\
$Q_{y}$ & Vertical tune & 18.16 \\
$\langle\beta\rangle_{x}[\mathrm{~m}]$ & Average $x$-betatron function & 111.18 \\
$\langle\beta\rangle_{y}[\mathrm{~m}]$ & Average $y$-betatron function & 106.63 \\
$\langle D\rangle_{x}[\mathrm{~m}]$ & Average dispersion & 0.936 \\
$\xi_{x, y}$ & $x, y$ chromaticity & 0.0 \\
$b_{x}[\mathrm{~cm}]$ & $x$ pipe size & 12.0 \\
$b_{y}[\mathrm{~cm}]$ & $y$ pipe size & 12.0 \\
$Q_{\perp}$ & Quality factor & 1.0 \\
$f_{r}[\mathrm{GHz}]$ & Resonance frequency & 1.0 \\
$R_{\perp}\left[\frac{M \Omega}{\mathrm{m}}\right]$ & Shunt impedance & 1.0 \\
$\beta=\sqrt{1-\gamma^{-2}}$ & Relativistic beta & 1.00 \\
$\eta=\gamma_{\mathrm{tr}}^{-2}-\gamma^{-2}$ & Phase slip factor & $3.455 \times 10^{-3}$ \\
$T_{\mathrm{rev}}[\mu \mathrm{s}]=\frac{C}{\beta c}$ & Revolution time & 23.06 \\
$f_{\mathrm{rev}}[\mathrm{Hz}]=\frac{1}{T_{\mathrm{rev}}}$ & Revolution frequency & $0.27 \times 10^{6}$ \\
$R[\mathrm{~m}]=C / 2 \pi$ & Machine radius & 1100 \\
$\omega_{c}[\mathrm{GHz}]=\frac{\beta c}{b_{x, y}}$ & Cutoff angular frequency & 2.50 \\
\hline \hline & & \\
\hline & & \\
\hline & &
\end{tabular}




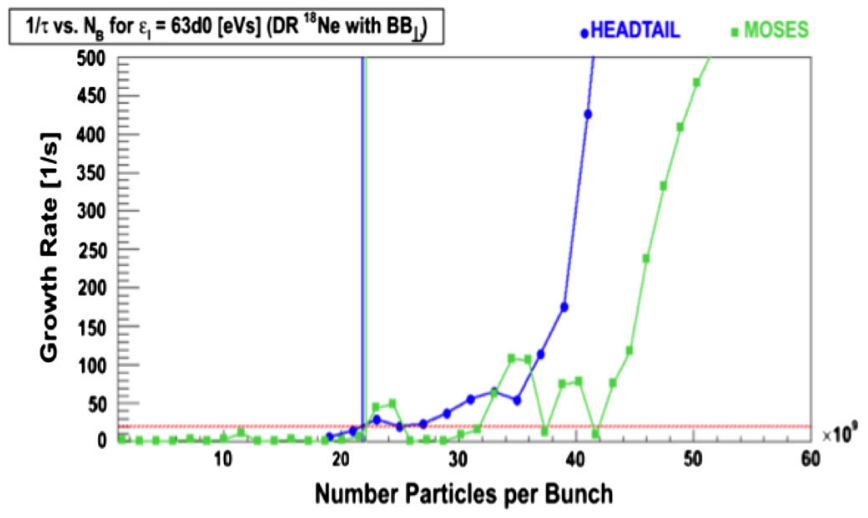

FIG. 48. Growth rate according to HEADTAIL (blue) and MOSES (green) as a function of bunch intensity.

a transport matrix. For the beta beam studies the possibility of bunches with ${ }^{18} \mathrm{Ne}$ and ${ }^{6} \mathrm{He}$ was added to the code.

The blue curve in Fig. 48 shows growth rates, $1 / \tau$, as a function of bunch populations, $N_{b}$, from HEADTAIL. Same as for MOSES the bunch intensity limit, $N_{b}^{\text {th }}$ (blue vertical line in Fig. 48) is reached when the rise time is shorter than allowed, i.e., $1 / \tau>(1 / \tau)^{\text {th }}$ (red dotted line).

\section{Decay ring scans}

With the three methods, mentioned above, we studied the effect on the bunch intensity limit, $N_{b}^{\text {th }}$, on the longitudinal bunch size by changing slightly the longitudinal emittance, $\varepsilon_{l}$, [Fig. 49(a)] and assuming $R_{\perp}=2 \mathrm{M} \Omega / \mathrm{m}$ and $(1 / \tau)^{\text {th }}=4001 / \mathrm{s}$ (see discussions below). We see that according to MOSES, HEADTAIL and the Coasting Beam equation (CB Eq.), see Eq. (24), increasing $\varepsilon_{l}$ the allowed number of ${ }^{18} \mathrm{Ne}$ per bunch increases but this also means an undesired increase in SF and momentum spread [also indicated in fig. 49(a)]. It is clear from Fig. 49(a) that the bunch intensity limit for ${ }^{18} \mathrm{Ne}, 3.4 \times 10^{12}$, is far out of reach when $R_{\perp}=2 \mathrm{M} \Omega / \mathrm{m}$ is assumed.

Since impedance could improve in modern machines compared to old accelerators, a scan over the shunt impedance was performed to see the impact on $N_{b}^{\text {th }}$ [Fig. 49(b)]. This was done for transverse broadband resonance impedance with all the parameters used shown in Table XIV.

Figure 49(b) shows that for a shunt impedance at the level of SPS, $R_{\perp}^{\mathrm{sps}}=20 \mathrm{M} \Omega / \mathrm{m}$, maximum number ${ }^{18} \mathrm{Ne}$ allowed per bunch, according to HEADTAIL, MOSES and CB Eq., is not more than $200 \times 10^{9}$. For $N_{b}=N_{b_{\text {sat }}}^{\text {nom }}=3.4 \times$ $10^{12}{ }^{18} \mathrm{Ne}$ per bunch $R_{\perp}<0.2 \mathrm{M} \Omega / \mathrm{m}$ is needed, which is easiest seen in Fig. 49(c). It could be argued that instabilities with the longest rise times should define $N_{b}^{\text {th }}$, i.e., $(1 / \tau)^{\text {th }} \rightarrow 0$. However in Figs. 49(b) and 49(c) an optimistic approach was taken. It was assumed that slow instabilities can be damped with sextupoles and octupoles, so $(1 / \tau)^{\text {th }}$ was defined to $4001 / \mathrm{s}$ for both MOSES and HEADTAIL. Also, defining $(1 / \tau)^{\text {th }} \rightarrow 0$ makes our approach more sensitive to systematic uncertainties. This is shown in Fig. 49(d), which is the same as Fig. 49(c) except that $(1 / \tau)^{\text {th }}=0.21 / s$. For some $R_{\perp}$ very slow instabilities for very small intensities are probed by our method which causes the discontinuity in the HEADTAIL results. Since this happens much more for MOSES than for HEADTAIL it is clear that this is due to systematics in the methods and the MOSES results were chosen not to be included in this plot.

Attempts to damp instabilities, and thereby allow more ions per bunch, have been made. Instabilities can be damped by avoiding resonances, i.e., making sure particles in the bunch oscillate with different frequencies. Tune spread in the bunch can be introduced by two different type of magnets; sextupole and octupole magnets. With sextupoles it is possible to introduce a tune dependence on the momentum offset. The achieved tune spreads follow $\Delta Q_{x, y}=\xi_{x, y} Q_{x, y} \frac{\Delta p}{p}$ where $\Delta p / p$ is the momentum spread and $\xi$ is the "chromaticity." By changing the chromaticity we investigated if the bunch intensity limit could be relaxed by increasing the sextupole magnet strength. It is known that the "rigid bunch mode" $(n=0)$ is stable for negative (positive) chromaticity below (above) transition, and unstable otherwise. So since $\eta>0$ for the DR and since $n=0$ is the most crucial mode (most likely to cause beam loss) we scanned $\xi \in[0,1]$ (where the unity is chosen as a normal chromaticity scale according to [127]). The simulation results are shown in Fig. 49(e) for $(1 / \tau)^{\text {th }}=0.21 / s$ since when damping is studied we do not assume any other damping. Also with damping it turned out that our methods were less vulnerable to systematics. It is evident that increasing the chromaticity would not help the intensity limit and it also confirms more instabilities for $\xi<0$. Octupole magnets can introduce a tune dependence on the oscillation amplitude of the bunch particles. The achieved tune spreads follow $\Delta Q_{x, y}=\frac{\partial Q_{x, x}}{\partial \varepsilon_{x, y}} a_{x}+\frac{\partial Q_{x, y}}{\partial \varepsilon_{y, y}} a_{y}$, where the single particle "action" $a_{\alpha}=\frac{\alpha^{2}+\alpha^{\prime 2} \beta_{\alpha}}{\beta_{\alpha}}, \alpha=x, y$, and $\frac{\partial Q_{x}}{\partial \varepsilon_{x}}, \frac{\partial Q_{y}}{\partial \varepsilon_{x}}$, $\frac{\partial Q_{x}}{\partial \varepsilon_{y}}$ and $\frac{\partial Q_{y}}{\partial \varepsilon_{y}}$ are the "amplitude detuning coefficients." By changing one of the amplitude detuning coefficients, $\frac{\partial Q_{y}}{\partial \varepsilon_{y}}$, we explored the dependence on the bunch intensity limit octupole magnets could have. The other coefficients are then fixed to $\frac{\partial Q_{x}}{\partial \varepsilon_{x}}=424.9 \mathrm{~m}^{-1}, \frac{\partial Q_{x, y}}{\partial \varepsilon_{y, x}}=-878.0 \mathrm{~m}^{-1}$. These values are taken from SPS measurements [128] where also $\frac{\partial Q_{y}}{\partial \varepsilon_{y}}=1155.0 \mathrm{~m}^{-1}$ was given. Amplitude detuning does damp instabilities in the DR, however for every instability damping, there is an unacceptable transversal emittance growth of the beam. This is shown in Fig. 49(f) where a scan over $\frac{\partial Q_{y}}{\partial \varepsilon_{y}} \in[0,2000] \mathrm{m}^{-1}$ was performed but no relaxing in $N_{b}^{\text {th }}$ could be claimed due to a parallel check in emittance growth. Even if as much as double transversal emittance growth was allowed the damping due to amplitude detuning had no impact. 


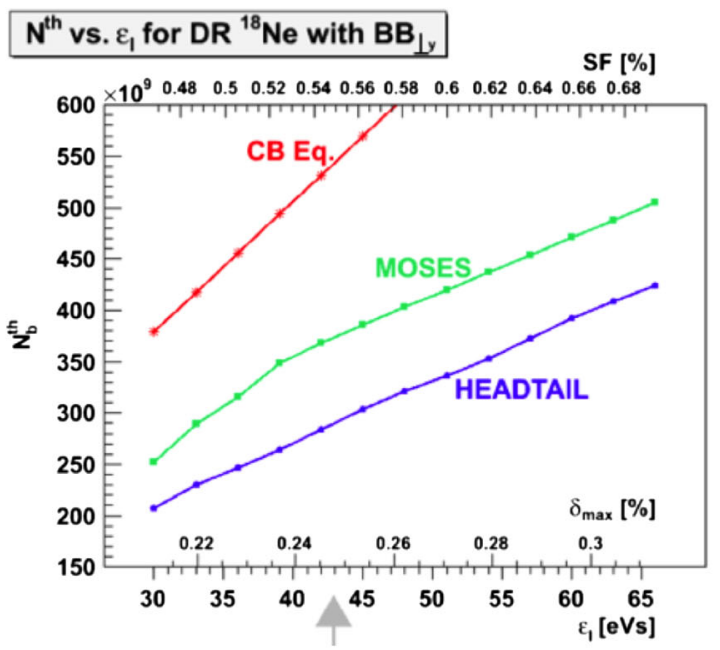

(a)

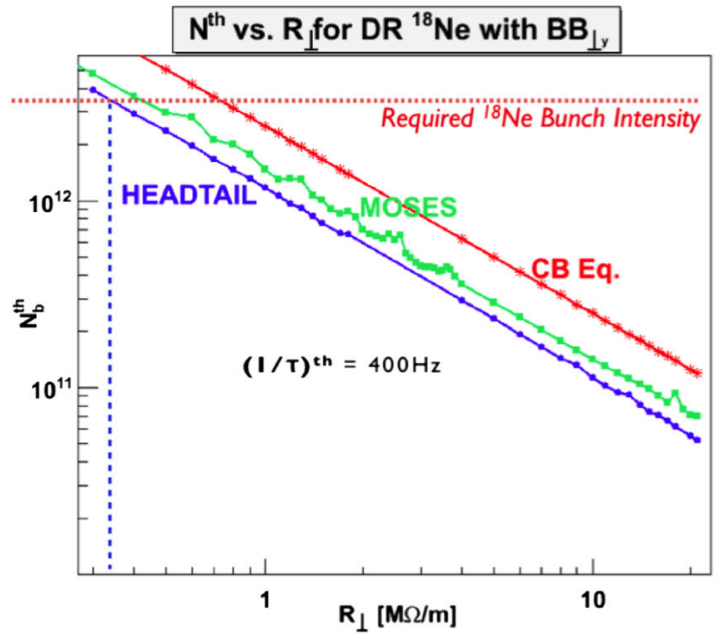

(c)

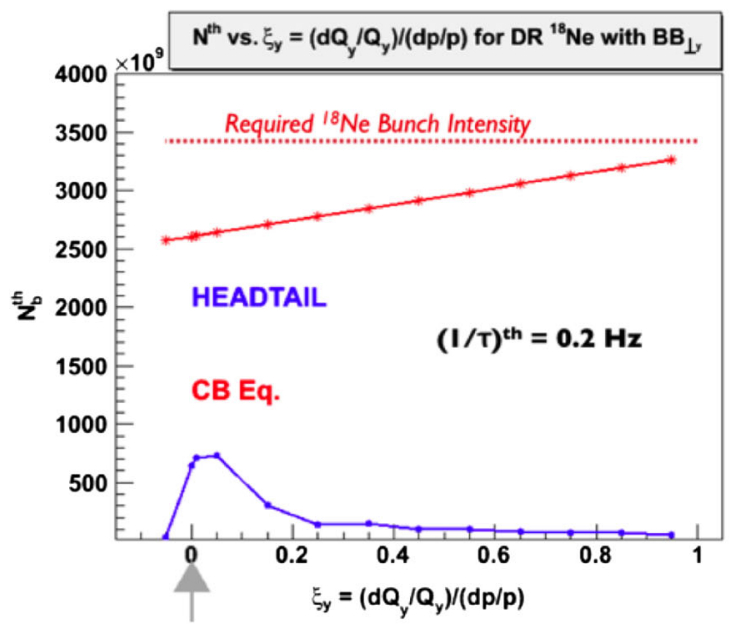

(e)

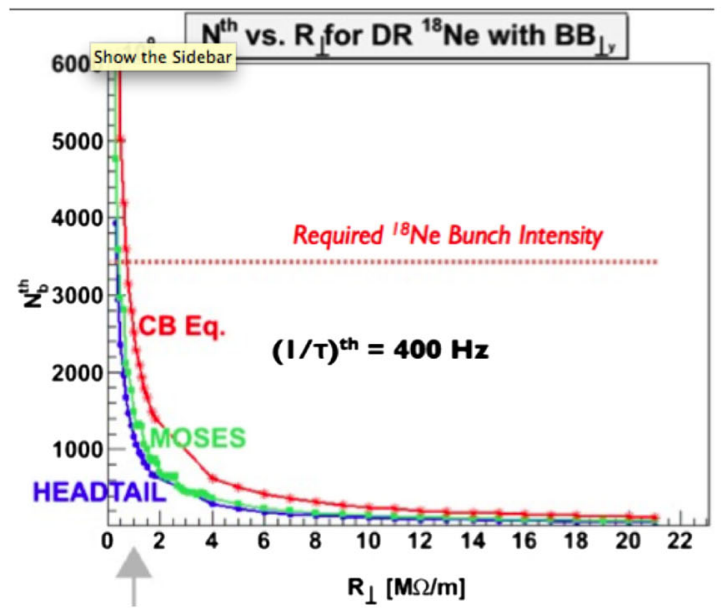

(b)

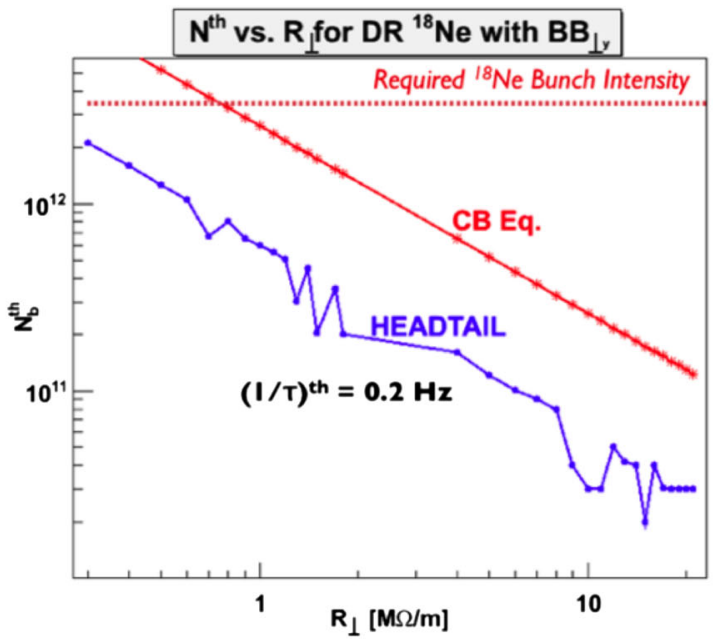

(d)

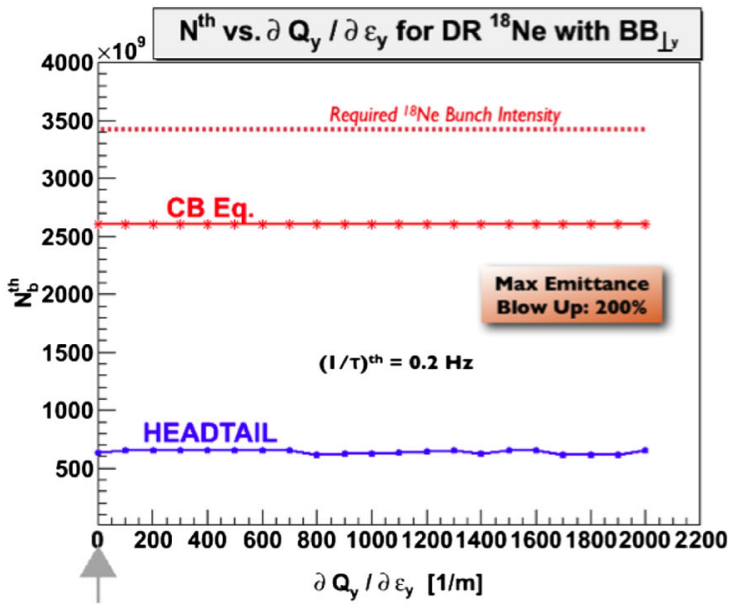

(f)

FIG. 49. $N_{B}^{\text {th }}$ as a function of (a) the longitudinal emittance and (b, c, d) the transversal shunt impedance according to the C.B. eq. (24), MOSES [125] and HEADTAIL [126]. Only the case for the neutrino emitter, ${ }^{18} \mathrm{Ne}$, in the decay ring is shown. With the log-log scale in (c) we see that $R_{\perp}<0.2 \mathrm{M} \Omega / \mathrm{m}$ is needed to allow $3.4 \times 10^{12}{ }^{18} \mathrm{Ne}$ per bunch. Instability dampings were attempted with chromaticity (e) and amplitude detuning (f), both without success. 


\section{Results for all ions}

From Eq. (24) we can extract that $N_{b}^{\text {th }} \propto \frac{A}{Z^{2}}$. This is used for estimations of bunch intensity limit for all the different radioactive ions investigated for the beta beams. For example, Fig. 49(d) gives that maximum $7 \times 10^{11}$ neon ions can populate each bunch in the DR assuming $R_{\perp}^{\mathrm{DR}}=1 \mathrm{M} \Omega / \mathrm{m}$. This gives then that maximum number of helium ions allowed per bunch is estimated with Eq. (24) to be $N_{b}^{\text {th }}=7 \times 10^{12}(6 / 18) /(2 / 10)^{2}=5.8 \times 10^{12}$. The required number of ions per bunch, $N_{b}^{\text {nom }}$, necessary to reach nominal neutrino flux, $\phi_{0}$, is for the DR given by

$$
N_{b}^{\mathrm{nom}}=\frac{\phi_{0} T_{C}}{N_{B} \ell_{\text {eff }} T_{\text {eff }}}\left(1-2^{-\frac{T_{C}}{\gamma_{1 / 2}}}\right)^{-1},
$$

where $T_{\text {eff }}=10^{7}$ seconds and all other parameters are given in Table XIV. We get from Table VIII that $N_{b}^{\text {nom }}=$ $4.7 \times 10^{12}$ number ${ }^{6} \mathrm{He}$ per bunch is necessary in the DR to achieve $\phi_{0}=2.9 \times 10^{18}$. So the bunch intensity limit for ${ }^{6} \mathrm{He}$, taking into account a transversal shunt impedance of $R_{\perp}=1 \mathrm{M} \Omega / \mathrm{m}$, is actually bigger than necessary; $N_{b}^{\text {th }} / N_{b}^{\text {nom }}=1.3$. This is however not the case for ${ }^{18} \mathrm{Ne}$ as can be seen in Table XV; only $20 \%$ of the required bunch intensities would be possible before collective effects would cause instabilities and beam loss. To use the excess of allowed ${ }^{6} \mathrm{He}$ ions and mitigate the deficiency of allowed ${ }^{18} \mathrm{Ne}$ it was suggested to aim for double ${ }^{6} \mathrm{He}$ intensity and half ${ }^{18} \mathrm{Ne}$ intensity. This turned out to have similar physics reach but only half the required bunch intensities would in that case be possible for both ions (see the middle setup in Table XV). One way to solve this would be to make the DR a two bore machine [129]. The larger deficiencies for high Q ion pair, ${ }^{8} \mathrm{~B}$ and ${ }^{8} \mathrm{Li}$, in the DR are however not as easily solved.

\section{Stability of beam, conclusions}

Collective effect studies for the beta beam decay ring and SPS have been performed taking into account

TABLE XV. The first four columns show the ion pair, assumed yearly neutrino fluxes, number of years for run and the $\theta_{13}$ sensitivities, from [98]. The fifth column shows single bunch ion intensity limit compared to nominal intensity necessary for the neutrino fluxes in the 2nd column. These results are based on transverse resonance broadband impedance studies for the DR (assuming $R_{\perp}=1 \mathrm{M} \Omega / \mathrm{m}$ ).

\begin{tabular}{lcccc}
\hline \hline Ions & $\nu$-flux $\left[10^{18}\right]$ & Years & $\left(\sin ^{2} \theta_{13}\right)_{\min }$ & $N_{b}^{\text {th }} / N_{b}^{\text {nom }}$ in DR \\
\hline${ }^{18} \mathrm{Ne}$ & $\phi_{0}=1.1$ & 5 & \multirow{2}{*}{$5 \times 10^{-4}$} & 0.20 \\
${ }^{6} \mathrm{He}$ & $\bar{\phi}_{0}=2.9$ & 5 & & 1.31 \\
${ }^{18} \mathrm{Ne}$ & $\phi_{0} / 2$ & 8 & $6 \times 10^{-4}$ & 0.41 \\
${ }^{6} \mathrm{He}$ & $\bar{\phi}_{0} \times 2$ & 2 & & 0.65 \\
${ }^{8} \mathrm{~B}$ & $\phi_{0} \times 5$ & 5 & $2 \times 10^{-4}$ & 0.15 \\
${ }^{8} \mathrm{Li}$ & $\bar{\phi}_{0} \times 5$ & 5 & & 0.15 \\
\hline \hline
\end{tabular}

transversal shunt impedance of $R_{\perp}^{\mathrm{DR}} \approx 1 \mathrm{M} \Omega / \mathrm{m}$ and $R_{\perp}^{\mathrm{SPS}}=20 \mathrm{M} \Omega / \mathrm{m}$ respectively. The DR study indicates that there will be large challenges due to requirements of seemingly low transverse broadband impedance. The bunch intensity limits in the SPS indicate that completely new solutions are necessary, possibly even a "green-field" beta beam.

The analysis software developed for these beta beam studies are general, object oriented and can easily be used for any beams [130].

\section{G. Conclusion}

Several aspects of the decay ring were studied in EUROnu. A new lattice was proposed enlarging the momentum compaction to push the intensity limits. An important advantage of this optics is to have very regular FODO lattices in the arcs, however more rf power and more dipoles are needed. In the case of a $\gamma=100$ decay ring, the required superconducting magnets with coil-free midplanes (arcs and injection section) may profit from existing technology; however development times are about 10 years. An alternative solution to the merging, barrier buckets is not promising in the range of parameters we use. A first design of the $40 \mathrm{MHz}$ cavities for the decay ring has been presented and a solution to the beam loading generated by the high beam intensities could be proposed. Collective effects, in particular the head tail effects, will not permit the decay ring with its present design to store the required ion intensities. Some solutions using multipoles to mitigate the head tail effects were investigated without success. However, with the new values measured of the neutrino oscillation angle $\theta_{13}$ the duty factor of the decay ring may be relaxed, see Sec. X below.

\section{POSSIBLE OPTIMIZATIONS IN VIEW OF BETTER PHYSICS}

The beta beam is essentially limited by the ion production and the beam stability. According to simulations, where only known losses are taken into account (decay losses, losses at the transfer from one machine to another, losses from merging in the decay ring), the presently achieved results for the production will give the number of neutrinos used by the physics evaluations to make the performance cost comparison. However, the fact that the oscillation angle $\theta_{13}$ is relatively large means that the suppression of atmospheric neutrinos could be relaxed. Preliminary simulations show that the duty factor of the decay ring (the distribution of the available intensity in the bunches) may be increased from $0.5 \%$ to $2 \%$ without significant loss in physics potential, assuming that the same intensity is delivered to the experiments. For the machines however, this may give better possibilities to have a stable beam by having more bunches with lower intensities, implying however that the bunching of the beta beam in 
all machines may need to be reconsidered. Losses will be reduced and the longitudinal merging at injection into the decay ring could be optimized to mitigate losses. This work remains to be done.

\section{COSTING}

The costing is an important ingredient to be able to select a way to proceed in preparing the strategic work to place (or not) these facilities in a global planning for the future.

The costing has been done in close collaboration between the three facilities to arrive at comparable costing scenarios. The available resource (technical expertise for this nontrivial task) has made it necessary to make many assumptions and apply scaling. For the beta beam we are grateful for the help we could get from different groups at CERN in spite of heavy workload for other activities. The result of the costing is a separate document, common for the three facilities, the Super Beam, the beta beam and the Neutrino Factory. Running costs of this facility are not part of the EUROnu study.

\section{CONCLUSION AND FOLLOW-UP}

The work on beta beams within EUROnu has essentially addressed the following topics: (i) design of a small storage ring for isotope production, (ii) development of a new approach to production using a molten salt loop, (iii) cross section measurement for the production of the high- $Q$ isotopes, (iv) collection of the produced isotopes, (v) possible use of barrier buckets to gain intensities at injection into the decay ring, (vi) simulations of the beam in the CERN machines: Q-scan in the PS and stability calculations in the decay ring and the SPS, (vii) development of a $60 \mathrm{GHz}$ electron cyclotron resonance source, (viii) tuning of the decay ring lattice for beam stability, (ix) optimization work on the decay ring and the cycling of the machines using the information on $\theta_{13}$. The work has shown that the beta beam is now feasible, however work remains to produce a technical design: a list of the most important items for the beta beam base-line option $\left({ }^{6} \mathrm{He}\right.$ and $\left.{ }^{18} \mathrm{Ne}\right)$ would be (i) a linac $160 \mathrm{MeV}, 1.2 \mathrm{MW}$, technical design, (ii) ${ }^{6} \mathrm{He}$ production full scale prototype with acceleration, (iii) ${ }^{18} \mathrm{Ne}$ full scale prototype, (iv) some adjustments of the magnetic field shape for the $60 \mathrm{GHz}$ ion source before design and construction, (v) technical design of the two RFQs and stripping, (vi) technical design of the RCS, (vii) technical design of the decay ring, including beam dumps and instrumentation, (viii) the PS injection system, (ix) PS space-charge measurements, (x) collective effects and rf studies of the SPS, (xi) technical design of the safety related equipment and radio protection studies in all machines, (xii) shielding in all machines.

\section{ACKNOWLEDGMENTS}

We acknowledge the financial support of the European Community under the European Commission Framework Program 7 Design Study: EUROnu, Project No. 212372. We would like to thank L. Maran, S. Marigo, A. Minarello, and L. Pranovi for the excellent technical support during the experiment at $\mathrm{LNL}$ for the measurements of ${ }^{8} \mathrm{Li}$ and cross section and we are grateful to Professor S. A. Goncharov for the theoretical ZR-KO-DWBA calculations and the fruitful discussions. We also acknowledge the help for costing of the different pieces of equipment, given by the expert groups at CERN (L. Bottura, G. de Rijk, E. Todesco, D. Tommasini, L. Tavian, A. Perin, J.-P. Burnet, J. Osborne, C. Waaijer, A. Kosmicki, L. Scibile, T. Pettersson, R. Catherall, H. Schmickler).

\section{APPENDIX A: TIME LINE FOR THE CONSTRUCTION OF A BETA BEAM}

To give a minimum time scale, the most time-demanding item should be pulled out; this is the decay ring magnet development. The construction of an ion linac and the RCS are also major projects. The other research topics could be made simultaneously. Initial R\&D is necessary, however the research topics are very different and can be done by different groups and laboratories simultaneously. The validity of this approach depends on available resources and how they can be deployed. The decay ring magnet design and model construction are estimated to 2.5 years: magnet full length prototype would be 2 years, tendering for series production (materials, superconductor and assembly contracts) would take 0.75 year. Tooling construction for production (superconductors and material deliveries) would be one year and to produce 152 magnets would take 3 years -in total 9.25 years from the start of the magnet design. The resistive magnets (series of $30 \mathrm{mag}-$ nets) would in principle be possible to produce simultaneously during 3 years. In Fig. 50 is shown the time line of the civil engineering work. It is assumed this can be done in parallel to magnet development.

Installation and commissioning time have been estimated to one year each.

In Table XVI, the estimated shortest time taken for different parts of the construction installation is shown.

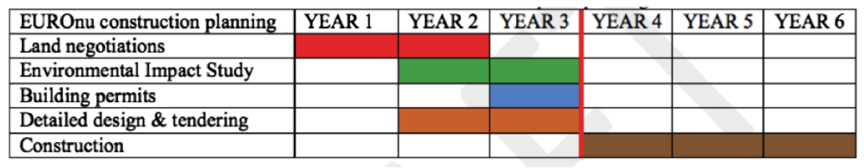

FIG. 50. Time line for the civil engineering of the beta beam facility. 
TABLE XVI. Beta-beam development and construction.

\begin{tabular}{lrc}
\hline \hline Initial R\&D & 3 & years \\
Decay ring magnets & 10 & years \\
Installation & 1 & year \\
Commissioning & 1 & year \\
Total & 15 & years \\
\hline \hline
\end{tabular}

\section{APPENDIX B: SAFETY ASPECTS FOR THE BETA BEAM}

The work on safety for the EUROnu beta beam is limited to a brief overview of aspects of safety and a list of presently known items to care about.

The long acceleration time, $3.6 \mathrm{~s}$ in the PS for both ${ }^{6} \mathrm{He}$ and ${ }^{18} \mathrm{Ne}$ and $3.6 \mathrm{~s}$ in the SPS for ${ }^{6} \mathrm{He}$ and $6 \mathrm{~s}$ for ${ }^{18} \mathrm{Ne}$, causes decay losses of about $50 \%$ for ${ }^{6} \mathrm{He}$ and $20 \%$ for ${ }^{18} \mathrm{Ne}$ before the beams are accelerated to $\gamma=100$.

For the PS and the SPS machines no specific modifications concerning the safety aspects can be seen. Some radio protection issues are specific to beta beams and have to be further investigated in particular localized losses; the overall radio protection has been studied for the RCS, the PS and the decay ring, see [131]. Areas of controlled access or remote handling are identified.

For the new machines, the cryogenic decay ring, the RCS and the new beam lines, it is assumed that the experience for the LHC can be used. A tentative list of items, valid for all machines, would be the following: (i) access system, (ii) fire detection system, (iii) evacuation alarm system, (iv) gas detection system, (v) oxygen deficiency hazard detection (cold machine), (vi) ventilation, (vii) electrical risks (powering interlocked with access system), (viii) cryogenic risks (cold machine), (ix) civil engineering and construction, $(\mathrm{x})$ lifting and handling.

Radio protection is well established at CERN for all environmental and legal issues. The following items need special attention:

Environment (dose to public): (i) stray radiation, (ii) releases of radioactivity into the environment (air and water)

Workers: (i) shielding, (ii) air and water activation induced radioactivity in accelerator components, (iii) activated fluids and contamination risk (closed circuits, etc.), (iv) optimized design of components (material composition), (v) optimized design for maintenance and repair, (vi) optimized handling of devices, remote handling, (vii) ventilation and pressure cascades.

A few items in the decay ring need special attention: (i) momentum collimators, (ii) superconducting magnets in radioactive environment, how to deal with losses in the arcs, maintenance (remote handling?), (iii) impedance considerations for absorbers and collimators.

A radiation monitoring system (like RAMSES [132]) is needed as well as buffer zones for cooldown and a repair workshop (access control, filters and fire proof areas have to be installed). Operational dosimetry systems and closed loops for cooling water, as well as remote systems for maintenance and remote handling need to be investigated as well and will add to the cost of the facility. An expensive item not to forget is provision for the dismantling and waste handling; these costs are in general high.

The ECR breeder in the production area creates high magnetic fields and high voltages. Microwaves and $\mathrm{x}$ rays have to be monitored and need controlled access. The safety conditions for the production in the ISOLDE area $\left({ }^{6} \mathrm{He}\right.$ and $\left.{ }^{18} \mathrm{Ne}\right)$ will follow already known procedures (ISOLDE at CERN and SPIRAL2 at GANIL). If the high$Q$ option is used the high pressure in the gas jet target would be a safety item, Lithium in contact with water has to be avoided, and the collection device has an oven of high temperature which also has to be put on the list of safety related items.

[1] P. Zucchelli, Phys. Lett. B 532, 166 (2002).

[2] M. Lindroos and M. Mezetto, Beta Beams: Neutrino Beams (Imperial College, London, 2010).

[3] L. Agostino, M. Buizza-Avanzini, M. Dracos, D. Duchesneau, M. Marafini, M. Mezzetto, L. Mosca, T. Patzak, A. Tonazzo, and N. Vassilopoulos, J. Cosmol. Astropart. Phys. 01 (2013) 024.

[4] E. Fernandez-Martinez, Nucl. Phys. B833, 96 (2010).

[5] F. P. An et al., Phys. Rev. Lett. 108, 171803 (2012).

[6] A. Lachaize and A. Tkatchenko, EURISOL DS Task Note No. 12-25-2008-0012, 2008.

[7] A. Chancé, Ph.D. thesis, Université Paris Sud, 2007.

[8] M. Vretenar, in Proceedings of the 25th International Linear Accelerator Conference LINAC10, Tsukuba, Japan (KEK, Tsukuba, Japan, 2010).

[9] R. Garoby, in Neutrino Town Meeting, 2012 (CERN, Geneva, 2012).

[10] FP7 INFRA-2007-2.1-01, Ref. 212343.

[11] J. Osborne, A. Kosmicki, and C. Waaijer, https://edms .cern.ch/document/1223890/1

[12] Final report of the EURISOL design study (2005-2009), a Design study for a European Isotope-Separation-OnLine radioactive ion beam facility, EU Contract No. 515768 (2009).

[13] C. Rubbia, A. Ferrari, Y. Kadi, and V. Vlachoudis, Nucl. Instrum. Methods Phys. Res., Sect. A 568, 475 (2006).

[14] T. Stora, in CERN Yellow Report No. CERN-2010-003, 2010.

[15] Final Report of the EURISOL Design Study, edited by J. Cornell (GANIL, Caen, France, 2009).

[16] H. Ravn and B. Alardyce, On-line Mass Separators, Treatise on Heavy-Ion Science, edited by D. Bromley (Plenum Press, New York, 1989).

[17] T. Stora, E. Noah, R. Hodak, T. Hirsch, M. Hass, V. Kumar, K. Singh, S. Vaintraub, P. Delahaye, H. FranbergDelahaye, M.-G. Saint-Laurent, and G. Lhersonneau, Europhys. Lett. 98, 32001 (2012). 
[18] ENDF Database, http://www-nds.iaea.org/exfor/endf .htm.

[19] T. Stora, E. Bouquerel, and J. Lettry, EURISOL-DS Technical Report No. TN03-25-2006-0003, 2006.

[20] L. Penescu, R. Catherall, J. Lettry, and T. Stora, Rev. Sci. Instrum. 81, 02 A906 (2010).

[21] M. Lagunas-Solar, Nucl. Instrum. Methods Phys. Res., Sect. B 69, 452 (1992).

[22] D. Williams, Oak-Ridge National Laboratory Technical Report No. ORNL/TM-2006/69, 2006.

[23] D. Williams, L. Toht, and K. Clarno, Oak-Ridge National Laboratory Technical Report No. ORNL/TM-2006/12, 2006.

[24] http://www.haynesintl.com/pdf/h3142.pdf.

[25] V. Parkhomchuk and A. Skrinsky, Sov. J. Nucl. Phys. 12, 3 (1981).

[26] D. Neuffer, Nucl. Instrum. Methods Phys. Res., Sect. A 532, 26 (1981).

[27] M. Schaumann, Reports No. CERN-THESIS-2009-128, EUROnu-WP4-004, 2009.

[28] E. Benedetto, in Proceedings of the 2nd International Particle Accelerator Conference, San Sebastián, Spain (EPS-AG, Spain, 2011).

[29] D. B. R. E. Marrs and E. Adelberger, Phys. Rev. C 8, 427 (1973).

[30] C. McClenahan and R. Segel, Phys. Rev. C 11, 370 (1975).

[31] C. Amsler et al., Phys. Lett. B 1, 667 (2008).

[32] O. Boine-Frankenheim, R. Hasse, F. Hinterberger, A. Lehrach, and P. Zenkevich, Nucl. Instrum. Methods Phys. Res., Sect. A 560, 245 (2006).

[33] D. Neuffer, Nucl. Instrum. Methods Phys. Res., Sect. A 585, 109 (2008).

[34] E. Benedetto, M. Schaumann, and J. Wehner, in Proceedings of the Workshop on European Strategy for Neutrino Physics (CERN Yellow Report No. CERN-2010-003, 2010).

[35] E. Benedetto, Report No. CERN ATS/Note/2011/094 TECH, 2011.

[36] M. Boucheron et al., Report No. CERN-PS-90-15-RF, 1990.

[37] V. Vardaci et al., LNL Annual Report 2020, p. 47.

[38] P. V. der Merwe, W. R. McMurray, and I. J. van Heerden, Nucl. Phys. A103, 474 (1967).

[39] N. Colonna et al., Nucl. Instrum. Methods Phys. Res., Sect. A 381, 472 (1996).

[40] The ROOT Team, http://root.cern.ch.

[41] S. Goncharov (private communication).

[42] G. R. Satchler, Direct nuclear reactions (Clarendon Press, Oxford, 1983), p. 833.

[43] B. Bayman and A. Kallio, Phys. Rev. 156, 1121 (1967).

[44] P. Kunz, The code DwUCK4.

[45] X. L. C. T. Liang and C. Cai, J. Phys. G 36, 085104 (2009).

[46] J. Dave and C. Gould, Phys. Rev. C 28, 2212 (1983).

[47] E. Fioretto et al., IEEE Trans. Nucl. Sci. 44, 1017 (1997).

[48] J. Schiffer, G. Morrison, R. Siemssen, and B. Zeidman, Phys. Rev. 164, 1274 (1967).

[49] S. Abramovich et al., J. Izv. Ross. Akad. Nauk 50, 65 (1986).
[50] T. Y. Hirsh, M. Hass, D. Berkovits, Y. Nir-El, L. Weissman, and F. de Oliveira, in Proc. Sci. Nufact08 (2008) 090.

[51] L. Weissman, D. Berkovits, I. Eliyahu, I. Gertz, A. Grin, S. Halfon, G. Lempert, I. Mardor, A. Nagler, A. Perry, J. Rodnizki, K. Dunkel, M. Pekeler, C. Piel, P. vom Stein, and A. Bechtold, in Proceedings of the 25th International Linear Accelerator Conference LINAC10, Tsukuba, Japan (Ref. [8]).

[52] S. Simakov, U. Fischer, V. Heinzel, and U. von Mollendorff, Wissenschaftliche Berichte FZKA Technical Report, 2002.

[53] R. Ferdinand and P. Bertrand, in Proceedings of the 25th International Linear Accelerator Conference LINAC10, Tsukuba, Japan (Ref. [8]), p. 16.

[54] T. Y. Hirsh, D. Berkovits, M. Hass, P Jardin, A. Pichard, M. L. Rappaport, Y. Shachar, and I. Silverman, J. Phys. Conf. Ser. 337, 012010 (2012).

[55] R. Geller, "Specified Elements of Plasma Physics Applied to Electron Cyclotron Resonance Ion Sources (ECRIS)," in Electron Cyclotron Resonance Ion Sources and ECR Plasmas, edited by B. Iop (Institute of Physics Publishing, Bristol, 1996) Chap. 1, pp. 1-146.

[56] T. Thuillier, J. Bouly, J. Curdy, E. Froidefond, and T. Lamy, in Proceedings of the 8th European Particle Accelerator Conference, Paris, 2002 (EPS-IGA and CERN, Geneva, 2002), pp. 1744-1746.

[57] T. Thuillier, T. Lamy, L. Latrasse, I. Izotov, A. Sidorov, V. Skalyga, V. Zorin, and M. Marie-Jeanne, Rev. Sci. Instrum. 79, $02 A 314$ (2008).

[58] T. Lamy, L. Latrasse, T. Thuillier, M. Marie-Jeanne, C. Trophime, F. Debray, P. Sala, J. Dumas, C. Fourel, J. Giraud, I. Izotov, A. Sidorov, V. A. Skalyga, and V. Zorin, "Bunched efficiency advanced study-60 GHz," EURISOL Design Study Final Report on Task 9: Beam Preparation (2009).

[59] O. Tarvainen, T. Ropponen, V. Toivanen, T. Kalvas, J. Komppula et al., in Proceedings of the VIIIth International Workshop on Strong Microwaves and Terahertz Waves: Sources and Applications (IAP RAS, Nizhny Novgorod, Russia, 2011).

[60] I. V. Izotov, A. V. Sidorov, V. A. Skalyga, V. G. Zorin, T. Lamy, L. Latrasse, and T. Thuillier, IEEE Trans. Plasma Sci. 36, 1494 (2008).

[61] V. Zorin, V. Skalyga, I. Izotov, S. Razin, A. Sidorov et al., in Proceedings of the XIXth International Workshop on ECR Ion Sources, 3402 ECRIS 2010, Grenoble, France.

[62] V. Zorin, V. Skalyga, I. Izotov, S. Razin, A. Sidorov et al., in Proceedings of the Transactions of Fusion Science and Technology, 2011 Vol. 59, p. 140.

[63] L. Latrasse, M. Marie-Jeanne, T. Lamy, T. Thuillier, J. Giraud, C. Fourel, C. Trophime, F. Debray, P. Sala, and J. Dumas, Rev. Sci. Instrum. 81, 02A324 (2010).

[64] H.-J. J. Schneider-Muntau, IEEE Trans. Magn. 18, 1565 (1982).

[65] F. Debray, J. Dumas, C. Trophime, and N. Vidal, Special issue on MT22 Proceedings [IEEE Trans. Appl. Supercond. 22, 4301804 (2012)].

[66] M. Marie-Jeanne, J. Jacob, T. Lamy, L. Latrasse, F. Debray, J. Matera, R. Pfister, and C. Trophime, in 
Workshop on ECR Ion Sources 3402 ECRIS 2010, Grenoble, France.

[67] T. Lamy, F. Debray, I. V. Izotov, M. Marie-Jeanne, A. V. Sidorov, V. A. Skalyga, P. Sortais, T. Thuillier, C. Trophime, E. Verney, N. Vidal, and V. G. Zorin, in Proceedings of the VIIIth International Workshop on Strong Microwaves and Terahertz. Waves: Sources and Applications (IAP RAS, Nizhny Novgorod, Russia, 2011).

[68] T. Lamy, M. Marie-Jeanne, P. Sortais, T. Thuillier, I. Izotov et al., in 13th International Workshop on Neutrino Factories, Super Beams and Beta Beams (NUFACT 11) (2011).

[69] T. Lamy, L. Latrasse, M. Marie-Jeanne, F. Debray, P. Sala, and C. Trophime, Special issue on MT22 Proceedings [IEEE Trans. Appl. Supercond. 22, 4101804 (2011)].

[70] B. Pardo, C. Trophime, S. Veys, and C. Prud'homme, LNCMI Grenoble Annual Report, 2011.

[71] M. H. Rashid, C. Mallik, and R. K. Bhandari, in XVIIIth International Conference on Cyclotrons and Their Applications (CYCLOTRONSO7) (2011).

[72] D. Leitner, M. L. Galloway, T. J. Loew, C. M. Lyneis, I. Castro Rodrigue, and D. S. Todd, Rev. Sci. Instrum. 79, $02 C 710$ (2008).

[73] M. Bakulin, A. Chirkov, G. Denisov, A. Eremeev, Z. Gasajniev et al., in 24th Joint Russian-German Meeting on ECRH and Gyrotrons (IAP RAS, Nizhny Novgorod, Russia, 2012).

[74] A. Bechtold and H. Podlech, in Proceedings of the 10th European Particle Accelerator Conference, Edinburgh, Scotland, 2006 (EPS-AG, Edinburgh, Scotland, 2006).

[75] F. Mazzolini, CAS, 2006.

[76] E. Benedetto, S. Damjanovic, S. Gilardoni, and E. Wildner, "BetaBeams in the CERN-PS: 2011 studies," Technical note (unpublished).

[77] M. Benedikt et al., EURISOL DS Task Note No. 12-252009-0014, 2007.

[78] S. Gilardoni and B. Goddard (private communication).

[79] R. Garoby, S. Gilardoni, B. Goddard, K. Hanke, M. Meddahi, and M. Vretenar, Conference Proceedings IPAC 2011 (CERN, Geneva, 2011).

[80] M. Magistris et al., Report No. CERN-SC-2009-051-RPTN, 2011.

[81] E. Benedetto, J. Phys. Conf. Ser. 408, 012059 (2013).

[82] G. Franchetti et al., Report No. GSI-Acc-Note-2004-05001, 2004.

[83] E. Benedetto, Report No. CERN-ATS-Note-2012-045MD, 2012.

[84] A. Chancé, Ph.D. thesis, Université Paris Sud, 2007.

[85] A. Chance and J. Payet, Proceedings of EPAC 2006, Edinburgh, Scotland, 2006, C060626, p. 1933.

[86] A. Chancé, Proc. Sci. (Nufact08) 088.

[87] M. Benedikt et al., Eur. Phys. J. A 47, 24 (2011).

[88] A. Chancé et al., Technical Report No. 12-25-2009-0019.

[89] A. Chance and J. Payet, in Proceedings of the 2nd International Particle Accelerator Conference, San Sebastián, Spain (Ref. [28]).

[90] E. Y. Wildner and F. W. Jones, in Proceedings of the 22nd Particle Accelerator Conference, Albuquerque, New Mexico (IEEE, New York, 2007).
[91] E. Bouquerel, A. Mereghetti, and V. Vlachoudis, eUROnu Note No. WP4-016, 2010.

[92] F. Borgnolutti, at the 9th Beta-beam Task Meeting, 2009.

[93] G. Leleux and P. Nghiem, Laboratoire National Saturne Technical Report, 1991.

[94] J. Payet, Laboratoire National Saturne Technical Report, 1993.

[95] J. Payet, Code beta, cea internal publication.

[96] S. Fartoukh and O. Brüning, LHC Project Report No. 501, 2001.

[97] J. Wei et al., in Proceedings of the Fifth European Particle Accelerator Conference, Sitges, Barcelona edited by S. Myers (CRC Press, 1996), p. 2704.

[98] A. Donini, Summary on Beta Beams, 2011.

[99] O. Brüning, "Beam parameters and definitions," in CERN LHC Design report.

[100] E. Metral, in 1st CARE-HHH-APD Workshop on Beam Dynamics in Future Hadron Colliders and Rapidly Cycling High-Intensity Synchrotrons, Geneva, Switzerland (CERN, 2004).

[101] S. van der Meer, Rev. Mod. Phys. 57, 689 (1985).

[102] S. Myers, Technical Report No. CERN-LEP-NOTE-344, 1981.

[103] S. Nagaitsev, in Proceedings of the 19th Particle Accelerator Conference, Chicago, Illinois, 2001 (IEEE, Piscataway, NJ, 2001), pp. 715-719.

[104] M. Benedikt and S. Hancock, Nucl. Instrum. Methods Phys. Res., Sect. A 550, 1 (2005).

[105] C. Carli, Technical Report No. CERN-PS-2001-073-AE, 2001.

[106] C. Carli and M. Chanel, Technical Report No. CERN-PS2002-019-AE, 2002.

[107] M. Benedikt, S. Hancock, and J. L. Vallet, Technical Report No. AB-Note-2003-080-MD, 2003.

[108] A. Chance and J. Payet, in Proceedings of the 21st Particle Accelerator Conference, Knoxville, 2005 (IEEE, Piscataway, NJ, 2005), pp. 3221-3223.

[109] Wolfram Research Inc., MATHEMATICA 8.0 (2007).

[110] A. Chance and S. Hancock, CERN Technical Report, 2006.

[111] D. C. Heinrich, C. Hansen, and A. Chance, AIP Conf. Proc. 1382, 211 (2011).

[112] European Commission, CORDIS, "Homepage of the Sixth Framework Package."

[113] C. Hansen, E. Wildner, and E. F. Martinez, in American Institute of Physics Conference Series (AIP, New York, 2010), pp. 455-458.

[114] R. Garoby, D. G. Grier, E. Jensen, A. Mitra, and R. L. Poirier, Technical Report No. CERN-PS-97-039-RF, 1997.

[115] H. Padamsee, J. Knobloch, and T. Hays, RF Superconductivity for Accelerators, Wiley Series in Beam Physics and Accelerator Technology (Wiley-VCH, New York, 2008).

[116] CST GmbH, MicrowaVe STUdio.

[117] R. Calaga, S. Belomestnykh, I. Ben-Zvi, and Q. Wu, IPAC'13, Shanghai, China C1205201 Vol. 2012, p. 003, WEPPC027.

[118] M. Magistris and M. Silari, Technical Report No. TIS2003-017-RP-TN, 2003. 
[119] J. Bruer and E. Todesco, IEEE Trans. Appl. Supercond. 19, 1191 (2009).

[120] J. Bruer (unpublished).

[121] K. Schindl, in CAS-CERN Accelerator School: Intermediate Course on Accelerator Physics (CERN, Geneva, 2006), p. 529.

[122] A. Chao (unpublished).

[123] Y. H. Chin, in Proceedings of the Joint US-CERN-JapanRussia School on Particle Accelerators, Montreux and CERN, Switzerland (World Scientific, 1998).

[124] G. Rumolo (private communication).

[125] Y. H. Chin, Report No. CERN-LEP-TH/88-05.

[126] G. Rumolo, Giovanni et al., Report No. CERN-SL-Note2002-036-AP.
[127] S. Y. Lee, Accelerator Physics (World Scientific, London, 2004), 2nd ed.

[128] R. Thomas et al., Report No. AB-Note-2003-025-MD.

[129] E. Wildner, Conference Proceedings for NEUTEL2011 M. Mezzetto (INFN, Padua, 2011).

[130] C. Hansen, in http://svnweb.cern.ch/world/wsvn/ bbcollective.

[131] S. Trovati, M. Magistris, and M. Silari, Reports No. CERN-SC-2008-070-RP-TN and No. CERN-DG2009-051-RP-TN

[132] G. Segura Millan, D. Perrin, and L. Scibile, 10th ICALEPCS International Conference on Accelerator and Large Experimental Physics Control Systems (JACOW, San Fransisco, 2005). 